\title{
Valuations on manifolds and integral geometry.
}

\author{
Semyon Alesker * \\ Department of Mathematics, Tel Aviv University, Ramat Aviv \\ 69978 Tel Aviv, Israel \\ e-mail: semyon@post.tau.ac.il
}

\begin{abstract}
One constructs new operations of pull-back and push-forward on valuations on manifolds with respect to submersions and immersions. A general Radon type transform on valuations is introduced using these operations and the product on valuations. It is shown that the classical Radon transform on smooth functions, and the well known Radon transform on constructible functions with respect to the Euler characteristic are special cases of this new Radon transform. An inversion formula for the Radon transform on valuations has been proven in a specific case of real projective spaces. Relations of these operations to yet another classical type of integral geometry, Crofton and kinematic formulas, are indicated.
\end{abstract}

\section{Contents}

0 Introduction. 2

0.1 A general overview of the article. . . . . . . . . . . . 2

0.2 A more detailed overview of the main results. . . . . . . . . . . . 4

0.3 Notation list. . . . . . . . . . . . . . . . . . . . . . 9

1 Background 10

1.1 Manifolds with corners. . . . . . . . . . . . . . . . . . . . . . 10

1.2 Construction of oriented blow up along a submanifold. . . . . . . . . . . 11

1.3 The Rumin differential operator. . . . . . . . . . . . . . . . . . . . 12

1.4 Generalized sections of vector bundles and wave fronts. . . . . . . . . 12

1.5 Normal cycles. . . . . . . . . . . . . . . . . . . . . . . 17

1.6 Valuations on manifolds. . . . . . . . . . . . . . . . . . . . 18

2 Exterior product of generalized valuations. 23

${ }^{*}$ Partially supported by ISF grant 701/08. 
3 Pull-back and push-forward of valuations. 25

3.1 Pull-back of smooth valuations under immersions. . . . . . . . . . . 25

3.2 Push-forward of smooth valuations under submersions. . . . . . . . . . . . 26

3.3 Pull-back of generalized valuations under submersions. . . . . . . . . . 27

3.4 Push-forward of generalized valuations under immersions. . . . . . . . . . . 28

3.5 Pull-back of generalized valuations under immersions. . . . . . . . . . . . 30

3.6 Push-forward of generalized valuations under submersions. . . . . . . . . . . 42

4 Valuations and integral geometry. 51

4.1 General Radon transform on valuations. . . . . . . . . . . . . . . . 51

4.2 Relations to the Gelfand style integral geometry. . . . . . . . . . . . . . . 60

4.3 Relations to the integral geometry of constructible functions. . . . . . . . . 61

4.4 Relations to the Crofton style integral geometry. . . . . . . . . . . . . . . . 69

5 Appendix.

\section{Introduction.}

\subsection{A general overview of the article.}

The theory of valuations on manifolds was introduced in [5], [6], [12], [7]; see also the survey of these results [8]. This theory generalizes in some direction the classical theory of valuations on convex subsets of $\mathbb{R}^{n}$ (see e.g. the surveys McMullen-Schneider [31] and McMullen [30]).

Let us describe a basic idea behind this new notion of a valuation on a manifold in an informal way. Valuation is a finitely additive complex valued functional defined on "nice" subsets of a smooth manifold $X$ with some extra properties to be discussed. By "nice" subsets we usually mean compact submanifolds with corners, while in the classical theory of valuations one considers the class of finite unions of convex compact subsets of $X=\mathbb{R}^{n}$.

The class of all finitely additive measures is too large and is probably out of control. In order to have a more restricted class of measures which still covers interesting examples from geometry and analysis, one could try to look for an extra condition of analytic nature which would tell how the measures of sets behave with respect to limits. In the classical measure theory of Lebesgue this condition is the countable additivity. As everybody knows, this condition turned out to be extremely useful in numerous situations.

In the new theory of valuations on manifolds we impose a rather different analytic condition (some version of continuity or smoothness). While on a general manifold this condition is somewhat technical (see [6]), it is basically motivated by the classical theory of valuations on convex sets in combination with some ideas from geometric measure theory: in the case of $X=\mathbb{R}^{n}$ the main condition is the continuity of the valuation $\phi$ on the class of convex compact sets with respect to the Hausdorff metric, namely if $\left\{K_{k}\right\}$ is a sequence of convex compact sets converging in the Hausdorff metric to another convex compact set $K$ then $\phi\left(K_{k}\right) \rightarrow \phi(K)$. To the best of our knowledge, this condition of continuity was first introduced and systematically studied by Hadwiger, see his book [24. Notice here also that results of geometric measure theory imply that the right generalization of this continuity condition to a more general class of non-convex sets is as follows: if $\left\{A_{k}\right\}$ is a sequence 
of "nice" compact subsets of a manifold $X$ such that the sequence of their normal cycles $\left\{N\left(A_{k}\right)\right\}$ (see Section 1.5 below) converges in the flat topology on currents to the normal cycle of a set $A$, then $\phi\left(A_{k}\right) \rightarrow \phi(A)$. On convex sets this kind of convergence is equivalent to the convergence in the Hausdorff metric.

We would like to emphasize that valuations may not be countably additive. Nevertheless smooth countably additive measures (i.e. those which locally can be written as $f\left(x_{1}, \ldots, x_{n}\right) d x_{1} \ldots d x_{n}$ with the function $f$ being $C^{\infty}$-smooth) are simplest examples of valuations in the new theory. Usually valuations cannot be defined on too broad class of sets, say on Borel sets. The most general class of sets for this theory is not very clear for the moment. Nevertheless if the manifold $X$ is real analytic then any smooth valuation can be naturally evaluated on any subanalytic relatively compact subset. It seems very likely that on a general smooth manifold $X$ valuations can naturally be evaluated also on compact subsets of positive reach (though a detailed proof has not appeared anywhere).

Another most basic example of a valuation is the Euler characteristic; definitely it is not countably additive, and it is not defined on say Borel sets. The classical theory of valuations on convex sets provides us with many more examples of valuations of geometric importance. Let us consider the Euclidean plane $\mathbb{R}^{2}$. The length of the boundary of a set is an example of a smooth valuation. In particular, it is defined on all compact submanifolds with corners and is continuous with respect to the Hausdorff metric on convex compact subsets.

More generally let us fix in $\mathbb{R}^{n}$ convex compact domains $A_{1}, \ldots, A_{k}, 1 \leq k \leq n-1$, with smooth positively curved boundaries. Consider the mixed volume

$$
\phi(K):=V(\underbrace{K, \ldots, K}_{n-k \text { times }}, A_{1}, \ldots, A_{n-k}) .
$$

Classically it is defined only for convex sets $K$ (see e.g. [38]), but in fact it can be naturally extended to all compact submanifolds with corners in $\mathbb{R}^{n}$, and this extension is a smooth valuation. For example if we take $A_{1}=\cdots=A_{k}$ to be the unit Euclidean ball then the valuation defined by 0.1 .1 is (up to a constant) the so called $(n-k)$-th intrinsic volume (in another terminology it is also called the Lipschitz-Killing curvature). In particular taking $n=2, k=1$, and $A_{1}$ being the Euclidean ball, we recover the length of the boundary of a subset of $\mathbb{R}^{2}$. There are also some other natural sources of valuations, for example coming from integral geometry.

Let us also mention that the space of translation invariant (continuous) valuations on convex subsets of $\mathbb{R}^{n}$ is a very classical object; this space almost coincides with the space of translation invariant valuations in the new setting of manifolds (more precisely, the former space contains the latter as a dense subspace). For $n \geq 2$ this space is infinite dimensional: it contains not only the Lebesgue measure, but also the Euler characteristic and all the mixed volumes. Nevertheless this space is not out of control, and much is known about it [24], [31], [30], [2], [9].

Let us denote by $V^{\infty}(X)$ the space of smooth valuations on a manifold $X$ (see Section 1.6 for a formal definition). It is naturally a Fréchet space. It has rich structures (see the survey [8]). In this article the multiplicative structure on $V^{\infty}(X)$ will be of a particular 
importance. The space $V^{\infty}(X)$ has a canonical product

$$
V^{\infty}(X) \times V^{\infty}(X) \rightarrow V^{\infty}(X)
$$

which is continuous, and $V^{\infty}(X)$ becomes a commutative associative algebra; the Euler characteristic $\chi$ is its unit element. The product on valuations was introduced first in [3] for so called polynomial valuations on convex subsets of $\mathbb{R}^{n}$, then extended to any smooth valuations on $\mathbb{R}^{n}$ in [5], and eventually in [12] it was shown that it is independent of the affine structure on $\mathbb{R}^{n}$, and hence extends to any manifold. A rather different construction of this product has been recently given in [11].

The first main goal of this article is to introduce the operations of pull-back and pushforward on valuations under smooth maps of manifolds. This is done under rather restrictive assumptions on the maps: they are assumed to be either submersions or immersions (more general cases will be discussed elsewhere). Nevertheless these cases are sufficient for some applications to integral geometry.

The second main goal of the article is to introduce a general Radon type transform on valuations. The construction of it involves the product on valuations and the operations of pull-back and push-forward mentioned above. We show that the classical Radon transform on smooth functions can be considered as a very special case of this new Radon transform (see Section 4.2). Moreover this new Radon transform generalizes (partly conjecturally) the well known and seemingly completely different Radon transform on constructible functions with respect to the Euler characteristic (see Section 4.3).

Eventually we prove in Theorem 4.3.3 an inversion formula for this new Radon transform on smooth valuations in a very concrete situation, generalizing the Khovanskii-Pukhlikov inversion formula [28] for the Radon transform with respect to the Euler characteristic on constructible functions on the real projective space $\mathbb{R} \mathbb{P}^{n}$.

In Section 4.4 we discuss the relations of the product, pull-back, and push-forward on valuations to yet another classical and quite different type of integral geometry: kinematic and Crofton type formulas.

Thus valuations on manifolds provide a general set up to unify different directions of integral geometry.

\subsection{A more detailed overview of the main results.}

Let us denote by $\mathcal{P}(X)$ the family of all compact submanifolds with corners of a manifold $X$. Smooth valuations are finitely additive functionals $\mathcal{P}(X) \rightarrow \mathbb{C}$ satisfying some additional conditions (see Section [1.6).

We have to recall few more general properties of valuations on manifolds (see e.g. the survey [8] ). We denote by $V_{c}^{\infty}(X)$ the subspace of $V^{\infty}(X)$ of compactly supported valuations. By [7] we have the integration functional

$$
\int_{X}: V_{c}^{\infty}(X) \rightarrow \mathbb{C}
$$

given by $\int_{X} \phi:=\phi(X)$. This is a linear functional which is continuous in appropriate topology on $V_{c}^{\infty}(X)$. 
We have a continuous bilinear map

$$
V_{c}^{\infty}(X) \times V^{\infty}(X) \rightarrow \mathbb{C}
$$

defined by $(\phi, \psi) \mapsto \int_{X} \phi \cdot \psi$ where under the integral we have the product on valuations mentioned above. By [7] (see also [13] for a simpler proof) this bilinear map is a perfect pairing, i.e. the induced map

$$
V^{\infty}(X) \rightarrow\left(V_{c}^{\infty}(X)\right)^{*}
$$

is injective and has a dense image in the weak topology. Thus we denote by $V^{-\infty}(X):=$ $\left(V_{c}^{\infty}(X)\right)^{*}$ and call it the space of generalized valuations. Via the map (0.2.1) we identify the space $V^{\infty}(X)$ of smooth valuations with its image in the space $V^{-\infty}(X)$ of generalized valuations:

$$
V^{\infty}(X) \subset V^{-\infty}(X)
$$

Let us describe now the relations between valuations and constructible functions. In the case of real analytic manifold $X$ this relation can be stated more elegantly. We will explain this case after considering first the more general case of a smooth manifold.

For a smooth manifold $X$ consider the natural map

$$
\Xi_{\mathcal{P}}: \mathcal{P}(X) \rightarrow V^{-\infty}(X)
$$

given by $P \stackrel{\Xi_{\mathcal{P}}}{\mapsto}[\phi \mapsto \phi(P)]$. This map is injective and the span of its image is dense in $V^{-\infty}(X)$ in the weak topology. Thus for any smooth manifold $X$ we have

$$
V^{\infty}(X) \subset V^{-\infty}(X) \supset \mathcal{P}(X) .
$$

In the special case when $X$ is a real analytic manifold it is more elegant to consider instead of $\mathcal{P}(X)$ the space $\mathcal{F}(X)$ of constructible functions. By definition $\mathcal{F}(X)$ consists of functions $f: X \rightarrow \mathbb{C}$ which are finite linear combinations with complex coefficients of integer valued functions $g: X \rightarrow \mathbb{Z}$ such that for any $m \in \mathbb{Z}$ the set $g^{-1}(m)$ is subanalytic, and the family of sets $\left\{g^{-1}(m)\right\}_{m \in \mathbb{Z}}$ is locally finite $\mathcal{F}(X)$ is an algebra over $\mathbb{C}$ with the pointwise product. Any smooth valuation can be naturally evaluated on a relatively compact subanalytic set, even if it is not a compact submanifold with corners (see [7]). Hence we have the map (also denoted by $\Xi_{\mathcal{P}}$ )

$$
\Xi_{\mathcal{P}}: \mathcal{F}(X) \rightarrow V^{-\infty}(X)
$$

given by $\sum_{i} \alpha_{i} \mathbb{1}_{P_{i}} \stackrel{\Xi_{\mathcal{P}}}{\mapsto}\left[\phi \mapsto \sum_{i} \alpha_{i} \phi\left(P_{i}\right)\right]$. By [7], Section 8.1, this map is injective and has a dense image in the weak topology. Thus for a real analytic manifold $X$ we have imbeddings of two linear dense subspaces analogous to (0.2.2)

$$
V^{\infty}(X) \subset V^{-\infty}(X) \supset \mathcal{F}(X)
$$

\footnotetext{
${ }^{1}$ Essentially this class of constructible functions was studied in detail in Ch. 9 of [27] where also the definition of a subanalytic subset can be found.
} 
The imbeddings (0.2.2), (0.2.3) are often very useful since various structures on valuations can be restricted to $\mathcal{P}(X)$ or, better to constructible functions $\mathcal{F}(X)$, where they can be interpreted in more familiar terms. For example the above mentioned product on $V^{\infty}(X)$ has the following interpretation. It was shown in [1] that $V^{-\infty}(X)$ has a partially defined product, i.e. two generalized valuations can be multiplied if they are in "generic position" to each other (the precise technical conditions are formulated on the language of wave front sets). For two smooth valuations this condition is satisfied automatically, and the product coincides with the above mentioned product on $V^{\infty}(X)$. On the other hand, the restriction of this partial product to $\mathcal{P}(X)$ is just the usual intersection of sets: more precisely given $P_{1}, P_{2} \in \mathcal{P}(X)$ which are transversal to each other then the product of their images in $V^{-\infty}(X)$ is well defined and is equal to the image of $P_{1} \cap P_{2}$; this was proved in [11. It is expected that for real analytic $X$ the restriction of the partial product on $V^{-\infty}(X)$ to $\mathcal{F}(X)$ coincides with the pointwise product on constructible functions provided the functions are in "generic position" to each other.

0.2.1 Remark. When talking on the map $\Xi_{\mathcal{P}}: \mathcal{P}(X) \rightarrow V^{-\infty}(X)$ for general smooth manifold $X$ we will often identify $P \in \mathcal{P}(X)$ with its indicator function $\mathbb{1}_{P}$ and thus write $\Xi_{\mathcal{P}}\left(\mathbb{1}_{P}\right)$.

After this reminder let us discuss the main results of this article. Let us discuss the pull-back and push-forward of valuations. In this introduction the discussion will be not completely rigorous and partly conjectural. The precise, but more technical, results can be found in the main text of the article. We will try however to indicate what is rigorous and what is not.

Let $f: M \rightarrow N$ be a smooth proper map of smooth manifolds. Under appropriate assumptions one should be able to define a linear map

$$
f_{*}: V^{\infty}(M) \rightarrow V^{-\infty}(N)
$$

called push-forward. The main idea is that $f_{*}$ should satisfy the usual (in comparison to the classical measure theory) property

$$
\left(f_{*} \phi\right)(P)=\phi\left(f^{-1}(P)\right)
$$

for any $P \in \mathcal{P}(N)$. On the technical level this is not well defined of course since the set $f^{-1}(P)$ may not belong to $\mathcal{P}(M)$. Nevertheless if $f$ is a submersion we get a well defined map $f_{*}: V^{\infty}(M) \rightarrow V^{\infty}(N)$ (see Section 3.2). For immersions the push-forward $f_{*}: V^{-\infty}(M)$ $\rightarrow V^{-\infty}(N)$ is defined rigorously in Section 3.4. One expects that $(f \circ g)_{*}=f_{*} \circ g_{*}$.

The pull-back map $f^{*}: V^{\infty}(N) \rightarrow V^{-\infty}(M)$ should be defined as the dual of $f_{*}$. Rigorously this has been done for immersions in Section 3.1, and for submersions in Section 3.3, One expects that $(f \circ g)^{*}=g^{*} \circ f^{*}$. Actually in order to define $f^{*}$ one does not have to assume that the map $f$ is proper.

Let us discuss these operations in some examples. First recall that smooth measures form a subspace of smooth valuations. Then the restriction of $f_{*}$ to smooth measures should coincide with the classical push-forward of measures; we have proven this when $f$ is either submersion or immersion. 
Under appropriate assumptions $f_{*}$ and $f^{*}$ should be extended by (sequential) continuity to larger subspaces of generalized valuations. Then we could restrict them to constructible functions (which are, perhaps, in "generic position" to the map $f$ ). Then on constructible functions $f^{*}$ should coincide with the usual pull-back of functions (this is proved rigorously for submersions in Section 3.3 and for closed imbeddings in Proposition 3.5.12).

The push-forward $f_{*}$ should coincide with the less trivial operation of integration with respect to the Euler characteristic along the fibers. It means the following: if $\mathbb{1}_{P}$ denotes the indicator function of a set $P \in \mathcal{P}(M)$ then

$$
\left(f_{*}\left(\mathbb{1}_{P}\right)\right)(y)=\chi\left(P \cap f^{-1}(y)\right)
$$

for any $y \in N$. If $P$ is in "generic position" with respect to $f$ then $f_{*}\left(\mathbb{1}_{P}\right)$ is a constructible function. This fact seems to be particularly technical to prove in full generality. We have proven it under quite restrictive assumptions (see Section 3.6 and the proof of Proposition 4.3.7). This fact is necessary for applications in integral geometry in Section 4.3.

Notice that pull-back of the Euler characteristic should be again the Euler characteristic whenever the pull-back is defined. We would like to have one more remark on $f^{*}$ when $f: M \rightarrow N$ is a submersion. In this case we have shown in Section 3.3 that $f^{*}: V^{-\infty}(N)$ $\rightarrow V^{-\infty}(M)$ is a continuous linear map. Let us restrict this map to smooth measures. The classical measure theory tells us that on measures there is no operation of pull-back. The new thing is that we can define the pull-back of a measure to be a (generalized) valuation. With an oversimplification, we have for a smooth measure $\mu$ on $N$ :

$$
\left(f^{*} \mu\right)(P)=\int_{N} \chi\left(P \cap f^{-1}(y)\right) d \mu(y) .
$$

Let us discuss now our applications of these constructions to integral geometry. A double fibration is a diagram

$$
X \stackrel{q_{1}}{\leftarrow} Z \stackrel{q_{2}}{\longrightarrow} Y
$$

of smooth submersive maps of smooth manifolds such that the map $q_{1} \times q_{2}: Z \rightarrow X \times Y$ is a closed imbedding. We assume in addition that $q_{2}$ is proper. Let us fix a smooth valuation $\gamma \in V^{\infty}(Z)$. We define a map which we call the Radon transform on valuations

$$
R_{\gamma}: V^{\infty}(X) \rightarrow V^{-\infty}(Y)
$$

by $R_{\gamma}(\phi)=q_{2 *}\left(\gamma \cdot q_{1}^{*} \phi\right)$ where the pull-back, push-forward, and the product are understood in the sense of valuations. Theorem 4.1.1 says that $R_{\gamma}$ is a well defined continuous linear operator. Here is a technical difficulty: though $\phi$ is a smooth valuation, the pull-back $q_{1}^{*} \phi$ is not smooth in general. Hence in order to define $R_{\gamma}(\phi)$ in general it was necessary to extend (partially) the push-forward map to generalized valuations.

Moreover Corollary 4.1.7 says that under certain assumption on the double fibration, $R_{\gamma}$ takes values in smooth valuations $V^{\infty}(Y)$ for any $\gamma \in V^{\infty}(Z)$, and it is a continuous linear map

$$
V^{\infty}(X) \rightarrow V^{\infty}(Y)
$$


Also under a similar assumption $R_{\gamma}$ extends (uniquely) by continuity to a linear map on generalized valuations

$$
V^{-\infty}(X) \rightarrow V^{-\infty}(Y) .
$$

Next we show that in the case when $\gamma$ is a smooth measure considered as a smooth valuation, the above Radon transform $R_{\gamma}$ can be considered as the classical Radon transform on smooth functions. More precisely, $R_{\gamma}$ uniquely factorizes in the following way:

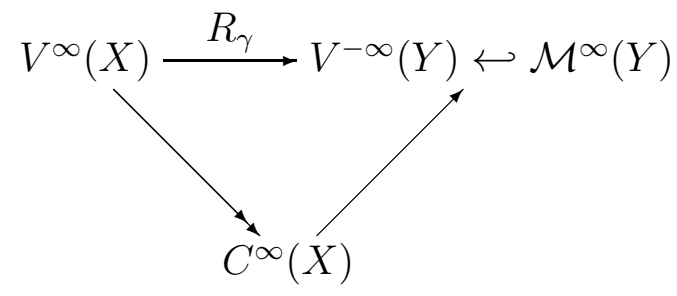

where the surjection $V^{\infty}(X) \rightarrow C^{\infty}(X)$ is the evaluation-on-points map, i.e.

$$
\phi \mapsto[x \mapsto \phi(\{x\})],
$$

and the map $C^{\infty}(X) \rightarrow \mathcal{M}^{\infty}(Y)$ is the classical Radon transform from the space $C^{\infty}(X)$ of smooth functions to the space $\mathcal{M}^{\infty}(Y)$ of smooth measures (see Section 4.2).

Let us consider another special case when $\gamma=\chi$ is the Euler characteristic. Assume that $R_{\chi}$ extends by continuity to a linear map $V^{-\infty}(X) \rightarrow V^{-\infty}(Y)$ (which is the case under appropriate assumptions; see Corollary 4.1.7). Assume in addition that $X, Y, Z, q_{1}, q_{2}$ are real analytic. Then we conjecture that $R_{\chi}(\mathcal{F}(X)) \subset \mathcal{F}(Y)$ (at least for "generic" constructible functions) and the map $R_{\chi}: \mathcal{F}(X) \rightarrow \mathcal{F}(Y)$ coincides with the Radon transform with respect to the Euler characteristic. Such kind of the Radon transform was considered by several authors under various modifications of the notion of constructibility: see [28], [29], [37]; in the earlier paper [39] the constructibility was considered in the sense of complex analytic geometry. We have not proven this conjecture in full generality, but some special cases of it were proven and used in Section 4.3 .

In Section 4.4 we describe relations of the product, pull-back, and push-forward on valuations to a very different type of integral geometry: kinematic and Crofton type formulas.

Finally let us describe our last main result: an inversion formula for the Radon transform on smooth valuations in a very concrete situation. Let $\mathbb{R} \mathbb{P}^{n}$ denote the real projective space, i.e. the space of lines through the origin in $\mathbb{R}^{n+1}$. Let $\mathbb{R} \mathbb{P}^{n \vee}$ denote the dual projective space, i.e. the space of hyperplanes through the origin in $\mathbb{R}^{n+1}$. Let $Z$ be the incidence variety, namely

$$
Z:=\left\{(l, H) \in \mathbb{R}^{n} \times \mathbb{R}^{n \vee} \mid l \subset H\right\} .
$$

Let $\mathbb{R} \mathbb{P}^{n} \stackrel{q_{1}}{\leftarrow} Z \stackrel{q_{2}}{\rightarrow} \mathbb{R}^{n \vee}$ be the double fibration where $q_{1}, q_{2}$ are the obvious projections. We have the Radon transform with respect to the Euler characteristic

$$
R_{\chi}: V^{\infty}\left(\mathbb{R}^{n}\right) \rightarrow V^{\infty}\left(\mathbb{R} \mathbb{P}^{n \vee}\right)
$$

defined as above, i.e. (taking into account that $\chi$ is the unit element) $R_{\chi}=q_{2 *} q_{1}^{*}$. Then $R_{\chi}$ is invertible up to a multiple of the Euler characteristic. More precisely consider the dual Radon transform

$$
R_{\chi}^{t}: V^{\infty}\left(\mathbb{R} \mathbb{P}^{n \vee}\right) \rightarrow V^{\infty}\left(\mathbb{R P}^{n}\right)
$$


defined similarly by $R_{\chi}^{t}:=q_{1 *} q_{2}^{*}$. Then we prove (Theorem 4.3.3) that for any $\phi \in V^{\infty}\left(\mathbb{R P}^{n}\right)$ one has

$$
(-1)^{n-1}\left(R_{\chi}^{t} \circ R_{\chi}\right) \phi=\phi+\frac{1}{2}\left((-1)^{n-1}-1\right)\left(\int_{\mathbb{R}^{n}} \phi\right) \cdot \chi .
$$

This inversion formula was motivated by the inversion formula for the Radon transform with respect to the Euler characteristic on "constructible" functions due to Khovanskii and Pukhlikov [28]. 2 The same inversion formula was generalized by Schapira [37] to functions constructible in the subanalytic sense.

0.2.2 Remark. Our proof of the inversion formula (0.2.5) on smooth valuations uses in fact the above mentioned result by Khovanskii and Pukhlikov and generalizes it in the following sense. The Radon transform $R_{\chi}$ can be extended by continuity to a linear map on generalized valuations

$$
R_{\chi}: V^{-\infty}\left(\mathbb{R P}^{n}\right) \rightarrow V^{-\infty}\left(\mathbb{R P}^{n \vee}\right)
$$

Similarly $R_{\chi}^{t}$ extends by continuity to generalized valuations.

If we restrict $R_{\chi}$ to the subspace of constructible functions in the sense of KhovanskiiPukhlikov (i.e. finite linear combinations of indicator functions of convex compact polytopes in $\mathbb{R P}^{n}$ ) then we show that $R_{\chi}$ coincides with the Radon transform with respect to the Euler characteristic. Since such functions are also dense in $V^{-\infty}\left(\mathbb{R P}^{n}\right)$, it suffices to prove the inversion formula (0.2.5) for them, and this was done by Khovanskii-Pukhlikov [28]. We conjecture that the restriction of $R_{\chi}$ to the broader class of subanalytic constructible functions also coincides with the Radon transform with respect to the Euler characteristic on this class. This would generalize then (a special case of) the result by Schapira [37] in the same way as we have generalized the result of Khovanskii-Pukhlikov.

Acknowledgements. I thank J. Bernstein for numerous useful discussions, and V. Milman for his attention to this work. I thank also V. Palamodov and S. Schochet for their explanations on wave front sets. I thank R. Schneider for some references.

\subsection{Notation list.}

- $V^{\infty}(X)$ - the space of smooth valuations on a manifold $X$.

- $V^{-\infty}(X)$ - the space of generalized valuations on a manifold $X$.

- $\mathcal{F}(X)$ - the space of constructible functions on $X$.

- $\mathcal{P}(X)$ - the family of compact submanifolds with corners in $X$.

- $R_{\gamma}$ - the Radon transform on valuations with the kernel $\gamma$.

- $\mathbb{P}_{+}(V)$ - the oriented projectivization of a real vector space (or a vector bundle) $V$, i.e. $(V \backslash\{0\}) / \mathbb{R}_{>0}$.

- $\Omega^{k}$ - either the vector bundle or the space of smooth $k$-forms.

- $\left|\omega_{X}\right|$ - the line bundle of densities on $X$.

- $\mathcal{M}^{\infty}(X)$ - the space of smooth measures (densities) on a manifold $X$.

\footnotetext{
${ }^{2}$ Actually their notion of constructibility was more restrictive one than what we have described above.
} 
- $C^{\infty}(X, \mathcal{E})$ - the space of smooth sections of a vector bundle $\mathcal{E}$ over $X$.

- $C^{-\infty}(X, \mathcal{E})$ - the space of generalized sections of a vector bundle $\mathcal{E}$.

- $W F(u)$ - the wave front of a generalized section $u$.

- $C_{\Lambda}^{-\infty}(X, \mathcal{E})$ - the space of generalized sections of a vector bundle $\mathcal{E}$ with wave front contained in a subset $\Lambda \subset T^{*} X$.

- $[[Z]]$ - a current associated to a submanifold $Z$.

- $\mathcal{D}_{k}(X)$ - the space of $k$-currents on $X$.

- $D$ - the Rumin differential operator on forms on a contact manifold.

- $\mathbb{P}_{X}$ - the spherical cotangent bundle of $X$, i.e. $\mathbb{P}_{X}:=\mathbb{P}_{+}\left(T^{*} X\right)$.

- $N(P)$ - the normal cycle of a subset $P$.

- $\mathbb{1}_{P}$ - the indicator function of a subset $P \subset X$.

- $T_{Z}^{*} X$ - the conormal bundle of a submanifold $Z \subset X$.

\section{Background}

\subsection{Manifolds with corners.}

1.1.1 Definition. A closed subset $P$ of an $n$-dimensional smooth manifold $X$ is called a submanifold with corners of dimension $k$ if any point $p \in P$ has an open neighborhood $U \ni p$ and a $C^{\infty}$-diffeomorphism $\phi: U \stackrel{\sim}{\rightarrow} \mathbb{R}^{n}$ such that for some $r \geq 0$

$$
\begin{array}{r}
\phi(p)=0, \\
\phi(P \cap U)=\mathbb{R}^{r} \times \mathbb{R}_{\geq 0}^{k-r} \times 0_{\mathbb{R}^{n-k}}
\end{array}
$$

where $0_{\mathbb{R}^{n-k}}$ is the zero element of $\mathbb{R}^{n-k}$. The set of submanifolds with corners is denoted by $\mathcal{P}(X)$.

The number $r$ is defined uniquely by the point $p$, but may depend on it. This $r$ is called type of a point $p$.

1.1.2 Example. 1. Any smooth submanifold of $X$ with or without boundary is a submanifold with corners.

2. A convex compact $n$-dimensional polytope $P \subset \mathbb{R}^{n}$ is a submanifold with corners if and only if $P$ is simplicial, namely every vertex has exactly $n$ adjacent edges. The vertices are precisely the points of type 0 .

A submanifold with corners $P \subset X$ has a natural finite stratification by locally closed smooth submanifolds as follows. For any integer $r, 0 \leq r \leq n$ let us denote by $S_{r}(P)$ the union of all points of type $r$. Then the $S_{r}(P)$ are locally closed smooth disjoint submanifolds of $X$ and

$$
P=\bigsqcup_{r=0}^{\operatorname{dim} P} S_{r}(P)
$$

This stratification of $P$ will be called canonical stratification. 
1.1.3 Definition. Let $P$ and $Q$ be two closed submanifolds with corners of $X$. We say that $P$ and $Q$ intersect transversally if each stratum of the canonical stratification of $P$ is transversal to each stratum of the canonical stratification of $Q$.

It is well known (se e.g. [6], Lemma 2.1.10) that if two closed submanifold with corners $P$ and $Q$ intersect transversally in the sense of Definition 1.1.3 then $P \cap Q$ is also a closed submanifold with corners.

\subsection{Construction of oriented blow up along a submanifold.}

Let $X$ be a smooth manifold and $Y \subset X$ be its closed submanifold (both $X$ and $Y$ are without corners or boundary). We remind here the so called oriented blow up of $X$ along $Y$ which is a manifold with boundary $\tilde{X}$ and a smooth map $\alpha: \tilde{X} \rightarrow X$.

Let $T_{Y} X:=\left(\left.T X\right|_{Y}\right) / T Y$ be the normal bundle of $Y$. Let $\mathbb{P}_{+}\left(T_{Y} X\right):=\left(T_{Y} X \backslash \underline{0}\right) / \mathbb{R}_{>0}$ be the oriented projectivization of it. Set theoretically, one defines

$$
\tilde{X}:=\mathbb{P}_{+}\left(T_{Y} X\right) \bigsqcup(X \backslash Y) .
$$

The map $\alpha: \tilde{X} \rightarrow X$ is equal to $I d$ on $X \backslash Y$, and to the natural projection $\mathbb{P}_{+}\left(T_{Y} X\right)$ $\rightarrow Y \subset X$ on $\mathbb{P}_{+}\left(T_{Y} X\right)$.

For the details of defining the smooth structure of $\tilde{X}$ we refer to [32], Ch. 5 (see also [11] for a special situation which suffices too for the purposes of this article). Here we give an idea how to do that for $X=\mathbb{R}^{n}=\mathbb{R}^{k} \times \mathbb{R}^{n-k}, Y=\mathbb{R}^{k} \times 0_{n-k} \subset \mathbb{R}^{n}$. Let us consider the imbedding

$$
X \backslash Y \rightarrow X \times \mathbb{P}_{+}\left(\mathbb{R}^{n-k}\right)=\mathbb{R}^{k} \times \mathbb{R}^{n-k} \times \mathbb{P}_{+}\left(\mathbb{R}^{n-k}\right)
$$

given by $(x, y) \mapsto(x, y, l)$ where $x \in \mathbb{R}^{k}, y \in \mathbb{R}^{n-k} \backslash \underline{0}$, and $l$ is the only oriented line in $\mathbb{R}^{n-k}$ passing through 0 and $y$ and oriented from 0 to $y$. Then define $\tilde{X}$ to be the closure of the image of $X \backslash Y$ under this map. The map $\alpha: \tilde{X} \rightarrow X$ is the restriction to $\tilde{X}$ of the natural projection $X \times \mathbb{P}_{+}\left(\mathbb{R}^{n-k}\right) \rightarrow X$.

It is not hard to see that the case of a general manifold $X$ can be deduced from this one by doing this construction in each coordinate chart and then gluing. Moreover any diffeomorphism $f: X \rightarrow X$ such that $f(Y)=Y$ has a unique lifting to a diffeomorphism $\tilde{f}: \tilde{X} \rightarrow \tilde{X}$ such that the diagram

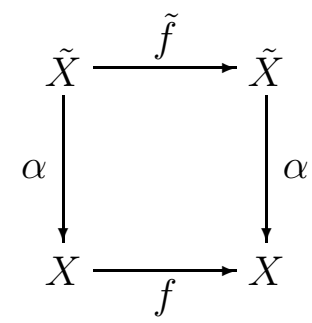

is commutative. 


\subsection{The Rumin differential operator.}

Let $M$ be a contact manifold of dimension $2 n-1$. Recall that this means that $M$ is given a smooth distribution of codimension 1 (i.e. a smooth field of hyperplanes in the tangent bundle) which is completely non-integrable. More explicitly, locally there exists a 1-form $\alpha$ such that the field of hyperplanes is equal to $\operatorname{Ker} \alpha$ with the property that $\alpha \wedge d \alpha^{n-1} \neq 0$. This form $\alpha$, which is called contact form, is unique up to multiplication by a non-vanishing smooth function.

A form $\omega \in \Omega^{*}(M)$ is called vertical if it vanishes on the contact distribution. Given a local contact form $\alpha$, the form $\omega$ is vertical if and only if $\omega \wedge \alpha=0$, or equivalently $\omega=\alpha \wedge \phi$ for some $\phi \in \Omega^{*}(M)$.

Given $\omega \in \Omega^{n-1}(M)$, there exists a unique vertical form $\omega^{\prime} \in \Omega^{n-1}$ such that $d\left(\omega+\omega^{\prime}\right)$ is vertical (see [34]). We define the projection operator $Q: \Omega^{n-1}(M) \rightarrow \Omega^{n-1}(M)$ by setting $Q \omega:=\omega+\omega^{\prime}$. This operator is a first order linear differential operator containing vertical forms in its kernel. The Rumin operator is the second order linear differential operator

$$
D:=d \circ Q: \Omega^{n-1}(M) \rightarrow \Omega^{n}(M) .
$$

The Rumin operator is the main ingredient in some differential complex, called Rumin-de Rham complex, whose cohomology is isomorphic to the de Rham cohomology [34]. We shall not use this isomorphism in the sequel.

\subsection{Generalized sections of vector bundles and wave fronts.}

We recall the definition and the main properties of the wave front of a generalized functions referring for more details to [25] or [23], Ch. VI.

Let $X$ be a smooth manifold (always countable at infinity, in particular paracompact). Let $\mathcal{E} \rightarrow X$ be a finite dimensional vector bundle; for definiteness we assume that $\mathcal{E}$ is a complex bundle though for real bundles the theory is exactly the same. We denote by $C^{\infty}(X, \mathcal{E})$ the space of $C^{\infty}$-sections of $\mathcal{E}$. It is a Fréchet space with topology of uniform convergence on compact subsets of $X$ of all partial derivatives. We denote by $C_{c}^{\infty}(X, \mathcal{E})$ the space of $C^{\infty}$-sections of $\mathcal{E}$ with compact support. Naturally $C_{c}^{\infty}(X, \mathcal{E})$ is a locally convex topological vector space with topology of (strict) countable inductive limit of Fréchet spaces. The inclusion

$$
C_{c}^{\infty}(X, \mathcal{E}) \hookrightarrow C^{\infty}(X, \mathcal{E})
$$

is continuous and has dense image.

Let us denote by $\left|\omega_{X}\right|$ the line bundle over $X$ of complex densities; thus the fiber of $\left|\omega_{X}\right|$ over a point $x \in X$ is equal to the one dimensional space of complex valued Lebesgue measures on the tangent space $T_{x} X$.

We have a separately continuous bilinear map

$$
C^{\infty}(X, \mathcal{E}) \times C_{c}^{\infty}\left(X, \mathcal{E}^{*} \otimes\left|\omega_{X}\right|\right) \rightarrow \mathbb{C}
$$

given by $(f, g) \mapsto \int_{X}\langle f, g\rangle$. This map is a non-degenerate pairing. In other words the induced map

$$
C^{\infty}(X, \mathcal{E}) \rightarrow\left(C_{c}^{\infty}\left(X, \mathcal{E}^{*} \otimes\left|\omega_{X}\right|\right)\right)^{*}
$$


is continuous, injective and has a dense image when the target space is equipped with the weak topology.

1.4.1 Definition. The space $\left(C_{c}^{\infty}\left(X, \mathcal{E}^{*} \otimes\left|\omega_{X}\right|\right)\right)^{*}$ is called the space of generalized sections of $\mathcal{E}$. It is denoted by $C^{-\infty}(X, \mathcal{E})$.

Thus $C^{\infty}(X, \mathcal{E}) \subset C^{-\infty}(X, \mathcal{E})$. Generalized sections of the trivial line bundle are called generalized functions. Generalized sections of $\mathcal{E}=\left|\omega_{X}\right|$ are called generalized densities.

The main technical tool in the following will be wave front sets of a generalized section of a vector bundle. Let us remind this notion following [25], Ch. VIII. For simplicity we will discuss the case of the trivial line bundle; the discussion easily extends to the general case. Moreover we assume that our manifold $X$ is $\mathbb{R}^{n}$; the considerations in the general case make use of local coordinates, and independence of the constructions of the local coordinates is proved in [25], Ch. VIII. Thus let $u \in C_{c}^{-\infty}\left(\mathbb{R}^{n}\right)$ be a compactly supported generalized function. Then its Fourier transform $\hat{u}$ is defined and is a continuous function on $\mathbb{R}^{n}$.

Let us define the subset $\mathcal{S}(u) \subset \mathbb{R}^{n}$ consisting of points $\eta$ with the property that there exists an open $\mathbb{R}_{>0}$-invariant neighborhood $V$ of $\eta$ such that for any $N \in \mathbb{N}$ there exists a constant $C_{N}$ such that

$$
|\hat{u}(\xi)| \leq C_{N}(1+|\xi|)^{-N} \text { for any } \xi \in V
$$

Denote by $\Sigma(u)$ the complement of $\mathcal{S}(u)$. Clearly $\Sigma(u)$ is a closed $\mathbb{R}_{>0}$-invariant subset of $\mathbb{R}^{n}$.

For a point $x \in \mathbb{R}^{n}$ define

$$
\Sigma_{x}(u):=\cap_{f} \Sigma(f \cdot u), f \in C_{c}^{\infty}\left(\mathbb{R}^{n}\right), f(x) \neq 0 .
$$

Finally one defines the wave front of $u$ by

$$
W F(u):=\left\{(x, \xi) \in \mathbb{R}^{n} \times\left(\mathbb{R}^{n} \backslash\{0\}\right) \mid \xi \in \Sigma_{x}(u)\right\}
$$

As we have mentioned, the definition of the wave front generalizes to any manifolds and to generalized sections of smooth vector bundles. Then $W F(u)$ is a subset of the cotangent bundle $T^{*} X$ with the zero section removed. Let us summarize the main properties of wave fronts relevant for our applications.

1.4.2 Proposition. ([25] or [23], Ch.VI§3).

Let $u \in C^{-\infty}(X, \mathcal{E})$.

(i) The wave front $W F(u)$ is a closed $\mathbb{R}_{>0}$-invariant subset of $T^{*} X \backslash \underline{0}$, where $\underline{0}$ denotes the zero-section.

(ii) $W F(u)=\emptyset$ if and only if $u$ is infinitely smooth.

(iii)

$$
W F(u \otimes v) \subset(W F(u) \times W F(v)) \cup(W F(u) \times \underline{0}) \cup(\underline{0} \times W F(v)) .
$$


Let us fix a closed $\mathbb{R}_{>0}$-invariant subset $\Lambda \subset T^{*} X \backslash \underline{0}$. Let us denote by $C_{\Lambda}^{-\infty}(X, \mathcal{E})$ the set of generalized sections of $\mathcal{E}$ whose wave front is contained in $\Lambda$. This is a linear subspace of $C^{-\infty}(X, \mathcal{E})$. Moreover $C_{\Lambda}^{-\infty}(X, \mathcal{E})$ is equipped with a locally convex linear topology. In the case of $\mathcal{E}$ being the trivial line bundle and $X=\mathbb{R}^{n}$ the topology is defined below; the general case is defined by covering $X$ by a system of local charts and trivializing $\mathcal{E}$ in each chart. Thus let us assume $X=\mathbb{R}^{n}$ and $\mathcal{E}$ is trivial. For any $N \in \mathbb{N}, \phi \in C_{c}^{\infty}\left(\mathbb{R}^{n}\right)$ and any closed $\mathbb{R}_{>0}$-invariant subset $V \subset \mathbb{R}^{n}$ such that

$$
\Lambda \cap(\operatorname{supp}(\phi) \times V)=\emptyset
$$

define the semi-norm on $C_{\Lambda}^{-\infty}(X, \mathcal{E})$

$$
\|u\|_{\phi, V, N}:=\sup _{\xi \in V}|\xi|^{N}|\widehat{\phi u}(\xi)| .
$$

Then one equips $C_{\Lambda}^{-\infty}(X, \mathcal{E})$ with the weakest locally convex topology which is stronger than the weak topology on $C^{-\infty}(X, \mathcal{E})$ and such that all semi-norms $\left\{\|\cdot\|_{\phi, V, N}\right\}$ are continuous. Notice that if $\Lambda=T^{*} X \backslash \underline{0}$ then $C_{\Lambda}^{-\infty}(X, \mathcal{E})=C^{-\infty}(X, \mathcal{E})$ as linear topological spaces.

Let $f: Y \rightarrow X$ be a smooth map, $\mathcal{E} \rightarrow X$ be a vector bundle. Then one has the obvious pull-back map on smooth sections

$$
f^{*}: C^{\infty}(X, \mathcal{E}) \rightarrow C^{\infty}\left(Y, f^{*} \mathcal{E}\right)
$$

It turns out that $f^{*}$ can be extended in a natural way to some generalized sections of $\mathcal{E}$ satisfying appropriate assumptions. Now we are going to discuss these assumptions referring for details to [23], Ch. VI $\S 3$.

Let us fix a closed conic subset $\Lambda \subset T^{*} X \backslash \underline{0}$. Let us consider the linear subspace $C_{\Lambda}^{-\infty}(X, \mathcal{E}) \subset C^{-\infty}(X, \mathcal{E})$ consisting of generalized sections of $\mathcal{E}$ with the wave front contained in $\Lambda$. The space $C_{\Lambda}^{-\infty}(X, \mathcal{E})$ is equipped with the locally convex linear topology defined above.

1.4.3 Definition. Let $\Lambda \subset T^{*} X \backslash \underline{0}$ be a closed conic subset. A smooth map $f: Y \rightarrow X$ is transversal to $\Lambda$ if for any $\xi \in T_{f(y)}^{*} X \cap \Lambda$ one has $d f_{y}^{*}(\xi) \neq 0$.

Note that a submersion is transversal to each $\Lambda \subset T^{*} X \backslash \underline{0}$.

1.4.4 Proposition. If a smooth map $f: Y \rightarrow X$ is transversal to a closed conic subset $\Lambda \subset T^{*} X \backslash \underline{0}$ then there is a unique linear sequentially continuous map, also called pull-back,

$$
f^{*}: C_{\Lambda}^{-\infty}(X, \mathcal{E}) \rightarrow C_{f^{*} \Lambda}^{-\infty}\left(Y, f^{*} \mathcal{E}\right)
$$

where

$$
f^{*}(\Lambda):=\left\{(y, \eta) \in T^{*} Y \mid \eta \in d f_{y}^{*}\left(\left.\Lambda\right|_{f(y)}\right)\right\}
$$

whose restriction to smooth sections of $\mathcal{E}$ is equal to the pull-back on smooth sections discussed above. 
Usually in the literature $f^{*}(\Lambda)$ is denoted by $d f^{*}(\Lambda)$. We will use the shorter notation as above throughout the article.

We denote by $T_{A}^{*} M$ the conormal bundle of any smooth submanifold $A \subset M$.

1.4.5 Remark. Let $Y$ be a closed submanifold of $X$, and $f: Y \rightarrow X$ be the identity imbedding. Then Proposition 1.4.4 says in particular that $f^{*} u$ is defined provided

$$
W F(u) \cap T_{Y}^{*} X=\emptyset .
$$

From Proposition 1.4.2 and Remark 1.4.5 one can easily deduce the following result on product of generalized sections (see [23], Ch. VI §3, Proposition 3.10). Below we denote by $s: \mathbb{P}_{X} \rightarrow \mathbb{P}_{X}$ the involution $s((x,[\xi]))=(x,[-\xi])$, and for a subset $Z \subset \mathbb{P}_{X}$ we denote by $Z^{s}$ the image $s(Z)$.

1.4.6 Proposition. Let $\mathcal{E}_{1}, \mathcal{E}_{2} \rightarrow X$ be two vector bundles. Let $\Lambda_{1}, \Lambda_{2}$ be closed conic subsets of $T^{*} X \backslash \underline{0}$. Let us assume that

$$
\Lambda_{1} \cap \Lambda_{2}^{s}=\emptyset
$$

Let us define a new subset $\Lambda \subset T^{*} X \backslash \underline{0}$ such that for any point $x \in X$

$$
\left.\Lambda\right|_{x}=\left.\left.\left(\left.\Lambda_{1}\right|_{x}+\left.\Lambda_{2}\right|_{x}\right) \cup \Lambda_{1}\right|_{x} \cup \Lambda_{2}\right|_{x}
$$

Then $\Lambda$ is also a closed conic subset, and moreover there exists a unique bilinear jointly sequentially continuous map

$$
C_{\Lambda_{1}}^{-\infty}\left(X, \mathcal{E}_{1}\right) \times C_{\Lambda_{2}}^{-\infty}\left(X, \mathcal{E}_{2}\right) \rightarrow C_{\Lambda}^{-\infty}\left(X, \mathcal{E}_{1} \otimes \mathcal{E}_{2}\right)
$$

whose restriction to smooth sections is the tensor product map.

We will need two further technical propositions. We denote by $\mathcal{D}(M)$ the space of currents on a manifold $M$ (if $M$ is oriented $\mathcal{D}(M)$ coincides with with the space of generalized differential forms).

1.4.7 Proposition. Let $A$ be a smooth submanifold of $M$. Let $\tilde{M}_{A}$ denote the oriented blow up of $M$ along $A$ (see Section 1.2). Let $f: \tilde{M}_{A} \rightarrow M$ be the natural map. Let $T \in \mathcal{D}(M)$. Then $f^{*} T \in \mathcal{D}\left(\tilde{M}_{A}\right)$ is defined provided

$$
T_{A}^{*} M \cap W F(T)=\emptyset .
$$

Proof. Let $x \in \tilde{M}_{A}$. If $x \notin f^{-1}(A)$ then clearly $f^{*} T$ is well defined in a neighborhood of $x$. Thus let us assume that $x \in f^{-1}(A)$. Let $\left.\xi \in W F(T)\right|_{f(x)}$. We have to show that $(d f)^{*} \xi \in T_{x}^{*} \tilde{M}_{A}$ does not vanish on $T_{x}\left(f^{-1}(A)\right)$, or, equivalently, that the restriction of $\xi$ to $d f\left(T_{x} f^{-1}(A)\right)$ does not vanish.

But $d f\left(T_{x} f^{-1}(A)\right)=T_{f(x)} A$, hence the assumption implies that the restriction of $\xi$ to $T_{f(x)} A$ does not vanish. Q.E.D. 
1.4.8 Proposition. Let $\tilde{M}_{A} \stackrel{f}{\longrightarrow} M \stackrel{g}{\longrightarrow} R$ be smooth maps where $f$ is the oriented blow up map as in Proposition 1.4.7, and $g$ is a submersion. Then $(g f)^{*} T$ is well defined provided the following condition is satisfied:

for any $a \in A$ and any $\zeta \in W F(T) \cap T_{g(a)}^{*} R$ the restriction of $\zeta$ to $d g\left(T_{a} A\right)$ does not vanish.

Proof. Since $g$ is a submersion, $g^{*} T$ is defined, and by Proposition 1.4 .4

$$
\left.W F\left(g^{*} T\right)\right|_{a} \subset\left(d g_{a}\right)^{*}\left(\left.W F(T)\right|_{g(a)}\right) .
$$

By Proposition 1.4.7 $f^{*}\left(g^{*} T\right)$ is defined provided

$$
T_{A}^{*} M \cap W F\left(g^{*} T\right)=\emptyset .
$$

By (1.4.3) this condition is satisfied provided that for any $a \in A$

$$
\left.\left(T_{A}^{*} M\right)\right|_{a} \cap\left(d g_{a}\right)^{*}\left(\left.W F(T)\right|_{g(a)}\right)=\emptyset
$$

The last condition is equivalent to the assumption of the proposition. Q.E.D.

Now let us discuss the push-forward of generalized sections. Let $f: Y \rightarrow X$ be a smooth proper map. Let $\mathcal{E} \rightarrow X$ be a smooth vector bundle as above. One can define the pushforward map

$$
f_{*}: C^{-\infty}\left(Y, f^{*}\left(\mathcal{E} \otimes\left|\omega_{X}\right|^{*}\right) \otimes\left|\omega_{Y}\right|\right) \rightarrow C^{-\infty}(X, \mathcal{E})
$$

as the dual map to

$$
f^{*}: C_{c}^{\infty}\left(X, \mathcal{E}^{*} \otimes\left|\omega_{X}\right|\right) \rightarrow C_{c}^{\infty}\left(Y, f^{*}\left(\mathcal{E}^{*} \otimes\left|\omega_{X}\right|\right)\right)
$$

Note that $f^{*}$ indeed takes compactly supported sections to compactly supported ones due to the properness of $f$.

1.4.9 Remark. Let us take $\mathcal{E}=\left|\omega_{X}\right|$. Then we get the push-forward map on generalized densities:

$$
f_{*}: C^{-\infty}\left(Y,\left|\omega_{Y}\right|\right) \rightarrow C^{-\infty}\left(X,\left|\omega_{X}\right|\right) .
$$

In the case when $f$ is a proper submersion, $f_{*}$ is integration along the fibers.

For a closed conic subset $W \subset T^{*} Y \backslash \underline{0}$ let us define a new conic subset

$$
f_{*} W:=\left\{(x, \eta) \in T^{*} X \backslash \underline{0} \mid \exists y \in f^{-1}(x) \text { such that }\left.\left(y, d f_{y}^{*}(\eta)\right) \in W\right|_{x} \cup\{0\}\right\} .
$$

The standard notation for $f_{*} W$ in the literature is $d f_{*} W$, but we prefer to abbreviate it as above. Since $f$ is proper, $f_{*} W$ is closed. One has the following result (see [23], Ch. VI $\S 3$, Proposition 3.9).

1.4.10 Proposition. Let $W \subset T^{*} Y \backslash \underline{0}$ be a closed conic subset. Then

$$
f_{*}: C_{W}^{-\infty}\left(Y, f^{*}\left(\mathcal{E} \otimes\left|\omega_{X}\right|^{*}\right) \otimes\left|\omega_{Y}\right|\right) \rightarrow C_{f_{*} W}^{-\infty}(X, \mathcal{E})
$$

is a sequentially continuous linear map. 
In the geometric measure theory, generalized sections of the space of forms $\Omega^{n-k}(X)$, $\operatorname{dim} X=n$, are called $k$-currents. An oriented compact $k$-submanifold (possibly, with corners) $Z \subset X$ defines a current $[[Z]] \in C^{-\infty}\left(X, \Omega^{n-k}\right)$ given by

$$
\phi \mapsto \int_{Z} \phi .
$$

The operator on currents dual to the de Rham differential $d$ is denoted by $\partial$. The space of $k$-currents is also denoted by $\mathcal{D}_{k}(X)$.

By Proposition 1.4.6 we have a partial product

$$
\cap: C^{-\infty}\left(X, \Omega^{n-k}\right) \times C^{-\infty}\left(X, \Omega^{n-l}\right)-\rightarrow C^{-\infty}\left(X, \Omega^{n-k-l}\right)
$$

extending the usual wedge product on smooth differential forms. For this reason we will denote sometimes the $\cap$-product by $\wedge$. If $Z_{1}, Z_{2} \subset X$ are oriented compact submanifolds (with corners) and $Z_{1}, Z_{2}$ intersect transversally then

$$
\left[\left[Z_{1}\right]\right] \cap\left[\left[Z_{2}\right]\right]=\left[\left[Z_{1} \cap Z_{2}\right]\right] .
$$

Also one has partially defined operations of pull-back and push-forward on currents as in Propositions 1.4.4 and 1.4.10.

\subsection{Normal cycles.}

Let us recall the definition of normal cycle of a compact submanifold with corners. This is a generalization of a unit conormal bundle of a smooth submanifold.

Let $X$ be an $n$-dimensional smooth manifold. For the simplicity of the notation we assume that $X$ is oriented though this assumption can be easily removed. Let $T X$ denote the tangent bundle of $X$, and $T^{*} X$ the cotangent bundle. Let $\mathbb{P}_{+}\left(T^{*} X\right)=: \mathbb{P}_{X}$ denote the oriented projectivization of the cotangent bundle $T^{*} X$, namely $\mathbb{P}_{X} \rightarrow X$ is a bundle over $X$ such that its fiber over a point $x \in X$ is equal to the quotient space $\left(T_{x}^{*} X \backslash\{0\}\right) / \mathbb{R}_{>0}$. Notice that if one fixes a Riemannian metric on $X$ then $\mathbb{P}_{X}$ is naturally identified with the spherical cotangent bundle of $X$. For this reason we call $\mathbb{P}_{X}$ the spherical cotangent bundle even if no metric is fixed.

Let $P \in \mathcal{P}(X)$ be a compact submanifold with corners. For any point $x \in P$ let $T_{x} P \subset$ $T_{x} X$ denote the tangent cone of $P$ at the point $x$. It is defined as follows:

$T_{x} P:=\left\{\xi \in T_{x} X \mid\right.$ there exists a $C^{1}-$ map $\gamma:[0,1] \rightarrow P$ such that $\gamma(0)=x$ and $\left.\gamma^{\prime}(0)=\xi\right\}$.

One can easily see that $T_{x} P$ is a convex polyhedral cone. If $P$ has no corners or boundary then $T_{x} P$ is the usual tangent space of $P$ at $x$. Let $\left(T_{x} P\right)^{\circ} \subset T_{x}^{*} X$ denote the dual cone. Recall that the dual cone $C^{o}$ of a convex cone $C$ in a linear space $W$ is defined by

$$
C^{o}:=\left\{y \in W^{*} \mid y(x) \geq 0 \text { for any } x \in C\right\} .
$$

By definition the normal cycle of $P$ is a subset $N(P) \subset \mathbb{P}_{X}$ defined by

$$
N(P):=\cup_{x \in P}\left(\left(T_{x} P\right)^{o} \backslash\{0\}\right) / \mathbb{R}_{>0} .
$$


It is well known (and easy to see) that $N(P)$ is $(n-1)$-dimensional submanifold (with singularities in general); the orientation of $X$ induces an orientation of $N(P)$; it is a cycle, i.e. $\partial N(P)=0$; and it is Legendrian with respect to the canonical contact structure on $\mathbb{P}_{X}$.

1.5.1 Remark. J. Fu [19] has defined normal cycle of a subanalytic subset of a real analytic manifold. An essentially equivalent notion of characteristic cycle of such a set was introduced independently using different tools by Kashiwara (see [27]). In this article we are not going to use this more complicated situation.

\subsection{Valuations on manifolds.}

The general reference for valuations on manifolds is the series of articles [5], [6], [12], [7], as well as the survey [8].

For simplicity of the notation we assume that $X$ is an oriented manifold, though this assumption can be easily removed, and all results and constructions can be generalized. A choice of orientation on $X$ induces in a standard way a choice of orientation on the normal cycle $N(P)$ for any $P \in \mathcal{P}(X)$ (we will not explain it here).

1.6.1 Definition. A smooth valuation is a map $\phi: \mathcal{P}(X) \rightarrow \mathbb{C}$ such that there exist a smooth $n$-form $\mu$ on $X$ (remind $n=\operatorname{dim} X$ ), and a smooth $(n-1)$-form $\omega$ on $\mathbb{P}_{X}$ such that

$$
\phi(P)=\int_{P} \mu+\int_{N(P)} \omega
$$

for any $P \in \mathcal{P}(X)$.

1.6.2 Remark. 1) Any $\phi$ of such form is a finitely additive functional on $\mathcal{P}(X)$.

2) In its present form the definition of smooth valuation might look unmotivated. The original definition of a smooth valuation from [6] was different but equivalent to this one, and behind it stay some non-trivial characterization results from the theory of valuations on convex sets and facts from geometric measure theory (see [6], [12]).

3) Various pairs of forms $(\mu, \omega)$ may define the same valuations. The pairs $(\mu, \omega)$ defining the zero valuation have been described by an explicit system of integral and differential equations by Bernig and Bröcker [14] as follows: $(\mu, \omega)$ defines the zero valuation if and only if

$$
\begin{aligned}
& \pi_{*} \omega=0, \text { and } \\
& D \omega+\pi^{*} \mu=0
\end{aligned}
$$

where $\pi: \mathbb{P}_{X} \rightarrow X$ is the natural projection, $D$ is the Rumin operator (see Section 1.3), $\pi_{*}$ is the operator of integration of a differential form along the fibers.

1.6.3 Example. 1) Any smooth measure on $X$ is a smooth valuation. Indeed take a pair $(\mu, 0)$.

2) The Euler characteristic $\chi$ is a smooth valuations. This is less trivial to see. This follows for example from an old theorem of Chern [17] who constructed explicitly a pair $(\mu, \omega)$ defining the Euler characteristic; his construction depends on a choice of an auxiliary Riemannian metric on $X$. A different proof was given in [6]. 
Thus the space $V^{\infty}(X)$ of smooth valuations is a quotient space of $\Omega^{n}(X) \oplus \Omega^{n-1}\left(\mathbb{P}_{X}\right)$, and hence it carries the natural linear Fréchet topology. Moreover $V^{\infty}(X)$ has a canonical filtration by closed subspaces

$$
V^{\infty}(X)=W_{0} \supset W_{1} \supset W_{2} \supset \cdots \supset W_{n}
$$

which can be described as follows. The last term $W_{n}$ is equal to the subspace of smooth measures. Each other subspace $W_{i}, 0 \leq i<n$, consist of smooth valuations which can be given by a pair $(\mu, \omega) \in \Omega^{n}(X) \oplus \Omega^{n-1}\left(\mathbb{P}_{X}\right)$ where $\omega$ satisfies the following condition: for any point $z \in \mathbb{P}_{X}$ and any $(n-1)$-dimensional subspace $L \subset T_{z} \mathbb{P}_{X}$ such that the intersection of $L$ with the tangent space at $z$ to the fiber $\pi^{-1}(\pi(z))$ is at least $(n-i)$-dimensional, the restriction of $\omega$ to $L$ vanishes. In particular $W_{0}=V^{\infty}(X)$.

Next we have the evaluation on points map $V^{\infty}(X) \rightarrow C^{\infty}(X)$, i.e. $\phi \mapsto[x \mapsto \phi(\{x\})]$. This linear map is onto, its kernel is equal to $W_{1}$. Thus the Fréchet space of smooth functions is canonically isomorphic to the Fréchet space $V^{\infty}(X) / W_{1}$. The following result was proved in [12], [7].

1.6.4 Theorem. There exists a canonical product $V^{\infty}(X) \times V^{\infty}(X) \rightarrow V^{\infty}(X)$ which is

(1) continuous;

(2) commutative and associative;

(3) the filtration $W$. is compatible with it:

$$
W_{i} \cdot W_{j} \subset W_{i+j}
$$

where we define $W_{j}=0$ for $j>n$;

(4) the Euler characteristic $\chi$ is the unit in the algebra $V^{\infty}(X)$;

(5) this product commutes with restrictions to locally closed submanifolds; in particular the evaluation on points map $V^{\infty}(X) \rightarrow C^{\infty}(X)$ is a homomorphism of algebras.

Let us describe a construction of the product on valuations following [11]. This is different from the construction given in [12], and it will be used in this article. First let us consider the natural map

$$
\pi_{X \times X}: \mathbb{P}_{X \times X} \rightarrow X \times X .
$$

Let us denote by $\mathbb{P}$ the preimage under this map of the diagonal in $X \times X$. Elements of $\mathbb{P}$ can be written in homogeneous coordinates $(x,[\xi: \eta])$ where $x \in X, \xi, \eta \in T_{x}^{*} X$. Let $\mathcal{M}$ denote the smooth submanifold of $\mathbb{P}$

$$
\mathcal{M}:=\{(x,[\xi: 0])\} \cup\{(x,[0: \eta])\} \cup\{(x,[\xi:-\xi])\}
$$

where clearly the union is disjoint. Let $\overline{\mathbb{P}}$ denote the oriented blow up of $\mathbb{P}$ along $\mathcal{M}$ (see Section 1.2). We have the maps $\Phi: \mathbb{P} \backslash \mathcal{M} \rightarrow \mathbb{P}_{X} \times_{X} \mathbb{P}_{X}$ given by $\Phi((x,[\xi: \eta]))=(x,[\xi],[\eta])$. Also we have a smooth map $p: \mathbb{P} \backslash \mathcal{M} \rightarrow \mathbb{P}_{X}$ given by $p(x,[\xi: \eta])=(x,[\xi+\eta])$. It is not hard to see that there exist unique smooth maps

$$
\begin{array}{r}
\bar{\Phi}: \overline{\mathbb{P}} \rightarrow \mathbb{P}_{X} \times_{X} \mathbb{P}_{X}, \\
\bar{p}: \overline{\mathbb{P}} \rightarrow \mathbb{P}_{X}
\end{array}
$$


extending $\Phi$ and $p$ respectively. Moreover $\bar{\Phi}$ and $\bar{p}$ are proper. Let

$$
q_{1}, q_{2}: \mathbb{P}_{X} \times_{X} \mathbb{P}_{X} \rightarrow \mathbb{P}_{X}
$$

be the obvious projections onto the first and second factors respectively. Let $s: \mathbb{P}_{X} \rightarrow \mathbb{P}_{X}$ be the involution given by $s(x,[\xi])=(x,[-\xi])$.

The following result was proved in [11].

1.6.5 Theorem. Let $X$ be an $n$-dimensional oriented manifold; let $\phi_{i} \in V^{\infty}(X)$ be represented by $\left(\omega_{i}, \mu_{i}\right) \in \Omega^{n-1}\left(\mathbb{P}_{X}\right) \times \Omega^{n}(X), i=1,2$. Then the product $\phi_{1} \cdot \phi_{2}$ is represented by

$$
\begin{aligned}
& \omega=\bar{p}_{*} \bar{\Phi}^{*}\left(q_{1}^{*} \omega_{1} \wedge q_{2}^{*} D \omega_{2}\right)+\omega_{1} \wedge \pi^{*} \pi_{*} \omega_{2} \in \Omega^{n-1}\left(\mathbb{P}_{X}\right), \\
& \mu=\pi_{*}\left(\omega_{1} \wedge s^{*}\left(D \omega_{2}+\pi^{*} \mu_{2}\right)\right)+\mu_{1} \wedge \pi_{*} \omega_{2} \in \Omega^{n}(X) .
\end{aligned}
$$

An important property of the product on valuations is a version of the Poincaré duality. To describe it let us denote first by $V_{c}^{\infty}(X)$ the subspace of $V^{\infty}(X)$ of compactly supported valuations. Then $V_{c}^{\infty}(X)$ admits a continuous integration functional

$$
\int: V_{c}^{\infty}(X) \rightarrow \mathbb{C}
$$

given by $\phi \mapsto \phi(X)$. Consider the bilinear map

$$
V^{\infty}(X) \times V_{c}^{\infty}(X) \rightarrow \mathbb{C}
$$

defined by $(\phi, \psi) \mapsto \int \phi \cdot \psi$. The following theorem was proved in [7], and a simpler proof was given in [13].

1.6.6 Theorem. This bilinear form is a perfect pairing. In other words, the induced map

$$
V^{\infty}(X) \rightarrow\left(V_{c}^{\infty}(X)\right)^{*}
$$

is injective and has a dense image (with respect to the weak topology on $\left.\left(V_{c}^{\infty}(X)\right)^{*}\right)$.

Define $V^{-\infty}(X):=\left(V_{c}^{\infty}(X)\right)^{*}$. Elements of this space are called generalized valuations. Thus by the last theorem $V^{\infty}(X)$ is identified with a dense subspace of $V^{-\infty}(X)$ :

$$
\Xi_{\infty}: V^{\infty}(X) \hookrightarrow V^{-\infty}(X)
$$

Let us denote by $\Theta: \Omega_{c}^{n}(X) \oplus \Omega_{c}^{n-1}\left(\mathbb{P}_{X}\right) \rightarrow V_{c}^{\infty}(X)$ (where the subscript $c$ stays for the compact support) the map given by

$$
(\Theta(\mu, \omega))(P)=\int_{P} \mu+\int_{N(P)} \omega .
$$

This map is onto. The dual map

$$
\Theta^{*}: V^{-\infty}(X) \rightarrow\left(\Omega_{c}^{n}(X)\right)^{*} \oplus\left(\Omega_{c}^{n-1}\left(\mathbb{P}_{X}\right)\right)^{*}=C^{-\infty}(X) \oplus C^{-\infty}\left(\mathbb{P}_{X}, \Omega^{n}\right)
$$


induces an injective closed imbedding. The result of Bernig and Bröcker (see Remark 1.6.2 (3)) implies that if a generalized valuation $\psi$ corresponds under this imbedding to a pair of currents $(C, T)$ then

$$
\begin{array}{r}
T \text { is a cycle, i.e. } \partial T=0 ; \\
T \text { is Legendrian; } \\
\pi_{*} T=\partial C .
\end{array}
$$

Conversely any pair of currents $(C, T)$ satisfying (1.6.5)-(1.6.7) corresponds to a unique generalized valuation. (Recall that $T$ is called Legendrian if for a contact form $\alpha$ one has $T \wedge \alpha=0$.) The described above product on smooth valuations has been extended in [11] to a partially defined product on generalized valuations. More precisely the following result was proved in [11]. (Below for a subset $\Lambda$ in the cotangent bundle of a manifold $M$ we denote by $\Lambda^{s}$ the image of $\Lambda$ under the antipodal involution $s$ acting on cotangent vectors to $M$.)

1.6.7 Theorem. Let $\Lambda_{1}, \Lambda_{2} \subset T^{*} X \backslash \underline{0}, \Gamma_{1}, \Gamma_{2} \subset T^{*} \mathbb{P}_{X} \backslash \underline{0}$ be closed conic subsets such that $d \pi_{*}\left(\Gamma_{i}\right) \subset \Lambda_{i}, i=1,2$. Let us assume that these subsets satisfy the following conditions:

(a) $\Lambda_{1} \cap \Lambda_{2}^{s}=\emptyset$.

(b) $\Gamma_{1} \cap\left(\pi^{*}\left(\Lambda_{2}\right)\right)^{s}=\emptyset$.

(c) $\Gamma_{2} \cap\left(\pi^{*}\left(\Lambda_{1}\right)\right)^{s}=\emptyset$.

(d) If $\left(x,\left[\xi_{i}\right], u_{i}, 0\right) \in \Gamma_{i}$ for $i=1,2$, then $u_{1} \neq-u_{2}$.

(e) If $(x,[\xi]) \in \mathbb{P}_{X}$ and

$$
\begin{aligned}
\left(u, \eta_{1}\right) & \left.\in \Gamma_{1}\right|_{(x,[\xi])} \\
\left(-u, \eta_{2}\right) & \left.\in \Gamma_{2}\right|_{(x,[-\xi])}
\end{aligned}
$$

then

$$
d \theta^{*}\left(0, \eta_{1}, \eta_{2}\right) \neq(0, l,-l) \in T_{(x,[\xi],[\xi])}^{*}\left(\mathbb{P}_{X} \times_{X} \mathbb{P}_{X}\right),
$$

where $\theta: \mathbb{P}_{X} \times_{X} \mathbb{P}_{X} \rightarrow \mathbb{P}_{X} \times_{X} \mathbb{P}_{X}$ is defined by $\theta\left(x,\left[\xi_{1}\right],\left[\xi_{2}\right]\right)=\left(x,\left[\xi_{1}\right],\left[-\xi_{2}\right]\right)$.

Then there is a unique jointly sequentially continuous bilinear map, called a partial product,

$$
V_{\Lambda_{1}, \Gamma_{1}}^{-\infty}(X) \times V_{\Lambda_{2}, \Gamma_{2}}^{-\infty}(X) \rightarrow V^{-\infty}(X)
$$

such that for any $\psi_{1}, \psi_{2} \in V^{\infty}(X)$ the product $\Xi_{\infty}\left(\psi_{1}\right) \cdot \Xi_{\infty}\left(\psi_{2}\right)$ exists (i.e. the conditions (a)-(e) are satisfied), and

$$
\Xi_{\infty}\left(\psi_{1}\right) \cdot \Xi_{\infty}\left(\psi_{2}\right)=\Xi_{\infty}\left(\psi_{1} \cdot \psi_{2}\right)
$$

In fact this partial product of generalized valuations can be described as follows. Consider again the maps

$$
\mathbb{P}_{X} \stackrel{\bar{p}}{\leftarrow} \overline{\mathbb{P}} \stackrel{\bar{\Phi}}{\rightarrow} \mathbb{P}_{X} \times_{X} \mathbb{P}_{X}
$$


as in (1.6.1)-(1.6.2). Assume that generalized valuations $\psi_{i} \in V_{\Lambda_{i}, \Gamma_{i}}^{\infty}, i=1,2$, are represented by pairs of currents $\left(C_{i}, T_{i}\right)$. Then the product $\psi_{1} \cdot \psi_{2}$ is represented by the pair $(C, T)$ where

$$
\begin{array}{r}
T:=(-1)^{n} \bar{p}_{*} \bar{\Phi}^{*}\left(q_{1}^{*} T_{1} \cap q_{2}^{*} T_{2}\right)+\pi^{*} C_{1} \cap T_{2}+T_{1} \cap \pi^{*} C_{2} \in C^{-\infty}\left(\mathbb{P}_{X}, \Omega^{n}\right), \\
C:=C_{1} \cap C_{2} \in C^{-\infty}(X) .
\end{array}
$$

The filtration $\left\{W_{\bullet}\right\}$ on smooth valuations $V^{\infty}(X)$ discussed above extends naturally to $V^{-\infty}(X)$ as follows (see [7], Section 7.3, for the details). Let us denote by $W_{i}\left(V^{-\infty}(X)\right)$ the closure of $W_{i}$ in the weak topology on $V^{-\infty}(X)$. Then by [7, Corollary 7.3.3, one has

$$
W_{i}\left(V^{-\infty}(X)\right) \cap V^{\infty}(X)=W_{i} .
$$

By the abuse of notation we will denote $W_{i}\left(V^{-\infty}(X)\right)$ by $W_{i}$.

Let us explain now the explicit relation of product on valuations to constructible functions assuming for simplicity of exposition that $X$ is a real analytic manifold. As we have explained in Section 0.2 of the introduction we have a canonical imbedding with dense image of the space of constructible functions

$$
\Xi_{\mathcal{P}}: \mathcal{F}(X) \hookrightarrow V^{-\infty}(X) .
$$

Let us assume that compact submanifolds with corners $P_{1}, P_{2}$ are transversal to each other. Then it was shown in [11] that the product of generalized valuations $\Xi_{\mathcal{P}}\left(\mathbb{1}_{P_{1}}\right)$ and $\Xi_{\mathcal{P}}\left(\mathbb{1}_{P_{2}}\right)$ is well defined, i.e. the assumptions of Theorem 1.6.7 are satisfied, and

$$
\Xi_{\mathcal{P}}\left(\mathbb{1}_{P_{1}}\right) \cdot \Xi_{\mathcal{P}}\left(\mathbb{1}_{P_{2}}\right)=\Xi_{\mathcal{P}}\left(\mathbb{1}_{P_{1} \cap P_{2}}\right) .
$$

The filtration $\left\{W_{\bullet}\left(V^{-\infty}(X)\right)\right\}$ induces on $\mathcal{F}(X)$ the filtration by codimension of support (see [7, Proposition 8.2.2).

The following technical lemma was proved in [11], Lemma 8.2, it will be useful later.

1.6.8 Lemma. Let $\xi \in V^{-\infty}\left(\mathbb{R}^{n}\right)$ be a generalized valuation given by a pair of currents $(C, T) \in \mathcal{D}_{n}\left(\mathbb{R}^{n}\right) \times \mathcal{D}_{n-1}\left(\mathbb{P}_{\mathbb{R}^{n}}\right)$. Let

$$
\Lambda \subset T^{*}\left(\mathbb{R}^{n}\right) \backslash \underline{0}, \Gamma \subset T^{*}\left(\mathbb{P}_{\mathbb{R}^{n}}\right) \backslash \underline{0}
$$

be closed conic subsets such that $\pi_{*}(\Gamma) \subset \Lambda$ and

$$
W F(C) \subset \Lambda, W F(T) \subset \Gamma .
$$

Then there exists a sequence of smooth valuations $\left\{\xi_{j}\right\} \subset V^{\infty}\left(\mathbb{R}^{n}\right)$ corresponding to currents $\left(C_{j}, T_{j}\right)$ such that

$$
\begin{array}{r}
C_{j} \rightarrow C \text { in } C_{\Lambda}^{-\infty}\left(\mathbb{R}^{n}\right), \\
T_{j} \rightarrow T \text { in } C_{\Gamma}^{-\infty}\left(\mathbb{P}_{\mathbb{R}^{n}}, \Omega^{n}\right) .
\end{array}
$$

In particular $\xi_{j} \rightarrow \xi$ in $V^{-\infty}(X)$. 


\section{Exterior product of generalized valuations.}

In this section we define the exterior product of two generalized valuations. The construction generalizes the construction of exterior product of two smooth valuations from [11] though we do not prove this fact here. Let $X_{1}, X_{2}$ be smooth manifolds (without boundary) of dimensions $n_{1}, n_{2}$ respectively. As usual we assume for simplicity of the notation that they are oriented. Set $X:=X_{1} \times X_{2}$. Let $\mathcal{M}_{1}, \mathcal{M}_{2} \subset \mathbb{P}_{X}$ be the submanifolds defined by

$$
\begin{aligned}
& \mathcal{M}_{1}=\left\{\left(x_{1}, x_{2} ;\left[\xi_{1}: 0\right]\right)\right\}, \\
& \mathcal{M}_{2}=\left\{\left(x_{1}, x_{2} ;\left[0: \xi_{2}\right]\right)\right\} .
\end{aligned}
$$

Let $F: \hat{\mathbb{P}} \rightarrow \mathbb{P}_{X}$ be the oriented blow-up map along $\mathcal{M}_{1} \sqcup \mathcal{M}_{2}$. Let

$$
\Phi: \hat{\mathbb{P}} \rightarrow \mathbb{P}_{X_{1}} \times \mathbb{P}_{X_{2}}
$$

be the only smooth map extending the map $\mathbb{P} \backslash\left(\mathcal{M}_{1} \sqcup \mathcal{M}_{2}\right) \rightarrow \mathbb{P}_{X_{1}} \times \mathbb{P}_{X_{2}}$ given by

$$
\left(x_{1}, x_{2} ;\left[\xi_{1}: \xi_{2}\right]\right) \mapsto\left(\left(x_{1},\left[\xi_{1}\right]\right) ;\left(x_{2},\left[\xi_{2}\right]\right)\right) .
$$

Let us also consider the diagrams

$$
\begin{gathered}
\mathbb{P}_{X_{1}} \stackrel{p_{1}}{\leftarrow} \mathbb{P}_{X_{1}} \times X_{2} \stackrel{i_{1}}{\hookrightarrow} \mathbb{P}_{X}, \\
\mathbb{P}_{X_{2}} \stackrel{p_{2}}{\longleftarrow} X_{1} \times \mathbb{P}_{X_{2}} \stackrel{i_{2}}{\longrightarrow} \mathbb{P}_{X}
\end{gathered}
$$

where $p_{1}, p_{2}$ are the obvious projections, and $i_{1}$ is given by $i_{1}\left(\left(x_{1},\left[\xi_{1}\right]\right), x_{2}\right)=\left(x_{1}, x_{2} ;\left[\xi_{1}: 0\right]\right)$, and $i_{2}$ is defined similarly.

Let us denote by $\tilde{p}_{i}: X_{1} \times X_{2} \rightarrow X_{i}, i=1,2$, the obvious projections. For $i=1,2$ let us define the spaces

$$
\mathcal{L}_{i}:=\left\{\left(C_{i}, T_{i}\right) \in \mathcal{D}_{n_{i}}\left(X_{i}\right) \oplus \mathcal{D}_{n_{i}-1}\left(\mathbb{P}_{X_{i}}\right) \mid T_{i} \text { is Legendrian, } \partial T_{i}=0, \pi_{X_{i} *} T_{i}=\partial C_{i}\right\} .
$$

Let $\psi_{i} \in V^{-\infty}\left(X_{i}\right), i=1,2$. Then $\psi_{i}$ is represented by by a unique pair $\left(C_{i}, T_{i}\right) \in \mathcal{L}_{i}$. In fact this gives a topological isomorphism between $V^{-\infty}\left(X_{i}\right)$ and $\mathcal{L}_{i}$ (see Section 8 of [11]). Let us consider

$$
(C, T) \in \mathcal{D}_{n_{1}+n_{2}}(X) \oplus \mathcal{D}_{n_{1}+n_{2}-1}\left(\mathbb{P}_{X}\right)
$$

defined by

$$
\begin{array}{r}
C:=C_{1} \otimes C_{2}, \\
T:=F_{*} \Phi^{*}\left(T_{1} \otimes T_{2}\right)+\left(\tilde{p}_{1} \circ \pi_{X}\right)^{*} C_{1} \cdot\left(i_{2 *} p_{2}^{*} T_{2}\right)+\left(i_{1 *} p_{1}^{*} T_{1}\right) \cdot\left(\tilde{p}_{2} \circ \pi_{X}\right)^{*} C_{2}
\end{array}
$$

where $\pi_{X}: \mathbb{P}_{X} \rightarrow X$ is the natural map.

2.1.9 Claim. The currents $C, T$ are well defined. Moreover the map $\left(\left(C_{1}, T_{1}\right),\left(C_{2}, T_{2}\right)\right) \mapsto$ $(C, T)$ defined by the formulas (2.1.12), (2.1.13) defines a bilinear jointly sequentially continuous map

$$
\left(\mathcal{D}_{n_{1}}\left(X_{1}\right) \oplus \mathcal{D}_{n_{1}-1}\left(\mathbb{P}_{X_{1}}\right)\right) \times\left(\mathcal{D}_{n_{2}}\left(X_{2}\right) \oplus \mathcal{D}_{n_{2}-1}\left(\mathbb{P}_{X_{2}}\right)\right) \rightarrow \mathcal{D}_{n_{1}+n_{2}}(X) \oplus \mathcal{D}_{n_{1}+n_{2}-1}\left(\mathbb{P}_{X}\right)
$$


Proof. The claim is obviously satisfied for $C$. To prove both parts of the claim for $T$, observe first of all that they are satisfied for the first summand in the definition of $T$ (i.e. in the right hand side of (2.1.13) $)$ since $\Phi$ is a submersion. The second and the third summands are symmetric, so let us consider only the third summand.

We have

$$
W F\left(i_{1 *} p_{1}^{*} T_{1}\right) \subset i_{1 *} p_{1}^{*}\left(T^{*} \mathbb{P}_{X_{1}} \backslash \underline{0}\right) .
$$

In order to describe the latter set, we will assume that $X_{1}, X_{2}$ are vector spaces. Also let us fix a point $A \in \mathbb{P}_{X}$ which we write in the form

$$
A=\left(x_{1}, x_{2} ;\left[\xi_{1}: \xi_{2}\right]\right)
$$

The fiber of $T^{*} \mathbb{P}_{X}$ over $A$ we can write as

$$
\left\{\left(x_{1}^{*}, x_{2}^{*} ; \zeta\right) \mid x_{1}^{*} \in T_{x_{1}}^{*} X_{1}, x_{2}^{*} \in T_{x_{2}}^{*} X_{2}, \zeta \in T_{\left[\xi_{1}: \xi_{2}\right]}^{*} \mathbb{P}_{+}\left(X^{*}\right)\right\}
$$

The fiber of $i_{1 *} p_{1}^{*}\left(T^{*} \mathbb{P}_{X_{1}} \backslash \underline{0}\right)$ over $A$ is non-empty if and only if $\xi_{2}=0_{X_{2}^{*}}$, and in this case this fiber is equal to

$$
\left(X_{1}^{*} \oplus\left\{0_{X_{2}^{*}}\right\} \oplus T_{\left[\xi_{1}: 0_{X_{2}^{*}}^{*}\right.}^{*} \mathbb{P}_{+}\left(X^{*}\right)\right) \backslash\{0\} .
$$

On the other hand the fiber over $A$ of $W F\left(\left(\tilde{p}_{2} \circ \pi_{X}\right)^{*} C_{2}\right)$ is contained in the fiber of $\left(\tilde{p}_{2} \circ\right.$ $\left.\pi_{X}\right)^{*} W F\left(C_{2}\right)$ which is contained obviously in

$$
\left\{0_{X_{1}^{*}}\right\} \oplus X_{2}^{*} \oplus\{0\}
$$

where the last zero belongs to $T_{\left[\xi_{1}: 0_{X_{2}^{*}}^{*}\right.}^{*} \mathbb{P}_{+}\left(X^{*}\right)$. But clearly the sets (2.1.14) and (2.1.15) satisfy the condition of disjointness. The claim follows. Q.E.D.

2.1.10 Claim. Assume that $\left(C_{i}, T_{i}\right) \in \mathcal{L}_{i}, i=1,2$. Let $C, T$ be defined by the formulas (2.1.12), 2.1.13) respectively. Then

(i) $T$ is Legendrian;

(ii) $\partial T=0$.

(iii) $\pi_{*} T=\partial C$.

Proof. Let us make first few observations.

(a) For compact submanifolds with corners $P_{i} \subset X_{i}, i=1,2$, let us consider the corresponding pairs of currents $\left(C_{i}, T_{i}\right):=\left(\left[\left[P_{i}\right]\right],\left[\left[N\left(P_{i}\right)\right]\right]\right)$. For such pairs one has

$$
\begin{array}{r}
C=\left[\left[P_{1} \times P_{2}\right]\right], \\
T=\left[\left[N\left(P_{1} \times P_{2}\right)\right]\right] .
\end{array}
$$

Clearly they satisfy the conditions (i)-(iii) of the claim, namely belongs to the subspace $\mathcal{L}$.

(b) Now in general the map

$$
\left(\left(C_{1}, T_{1}\right),\left(C_{2}, T_{2}\right)\right) \mapsto(C, T)
$$

is jointly sequentially continuous. 
(c) The subspace $\mathcal{L} \subset \mathcal{D}_{n_{1}+n_{2}}(X) \oplus \mathcal{D}_{n_{1}+n_{2}-1}\left(\mathbb{P}_{X}\right)$ is closed in the weak topology.

(d) There is a natural isomorphism of topological vector spaces $V^{-\infty}\left(X_{i}\right) \simeq \mathcal{L}_{i}, V^{-\infty}(X) \simeq$ $\mathcal{L}$ (see Section 8 of [11]).

These observations and Corollary 1.3 in [10] imply that any jointly (in fact, even separately) sequentially continuous map $\mathcal{L}_{1} \times \mathcal{L}_{2} \rightarrow \mathcal{D}_{n_{1}+n_{2}}(X) \oplus \mathcal{D}_{n_{1}+n_{2}-1}\left(\mathbb{P}_{X}\right)$ which maps pairs of indicator functions of compact submanifolds with corners to $\mathcal{L}$, has image contained in $\mathcal{L}$. Q.E.D.

2.1.11 Claim. The exterior product of constructible functions considered as generalized valuations coincides with the usual product of constructible functions.

Proof. This immediately follows from the construction. Q.E.D.

\section{Pull-back and push-forward of valuations.}

\subsection{Pull-back of smooth valuations under immersions.}

Let

$$
f: X^{n} \rightarrow Y^{m}
$$

be an immersion of smooth manifolds, $n<m$. Let us define the pull-back map on smooth valuations

$$
f^{*}: V^{\infty}(Y) \rightarrow V^{\infty}(X)
$$

as follows. By the sheaf property of smooth valuations (see [6], Theorem 2.4.10) it is enough to define pull-back locally on $X$. Then we may assume that $f$ is a closed imbedding. Then define

$$
\left(f^{*} \phi\right)(P):=\phi(f(P))
$$

for any $P \in \mathcal{P}(X)$. The following claim is obvious.

3.1.1 Claim. If $f: X \rightarrow Y$ is a smooth immersion then

$$
f^{*}: V^{\infty}(Y) \rightarrow V^{\infty}(X)
$$

is a continuous linear operator.

Actually we will need yet another description of the pull-back under immersions. Let us assume that $X \subset Y$ is a closed submanifold. Let us consider the obvious maps

$$
X \stackrel{q}{\leftarrow} \mathbb{P}_{+}\left(T_{X}^{*} Y\right) \stackrel{\theta}{\hookrightarrow} \mathbb{P}_{Y} .
$$

Observe first that $X \times_{Y} \mathbb{P}_{Y}$ is a smooth submanifold of $\mathbb{P}_{Y}$. Moreover the oriented projectivization $\mathbb{P}_{+}\left(T_{X}^{*} Y\right)$ of the conormal bundle of $X$ in $Y$ is a closed submanifold in $X \times_{Y} \mathbb{P}_{Y}$. Let us denote by $X \widetilde{\times_{Y}} \mathbb{P}_{Y}$ the oriented blow up of $X \times_{Y} \mathbb{P}_{Y}$ along $\mathbb{P}_{+}\left(T_{X}^{*} Y\right)$. Let

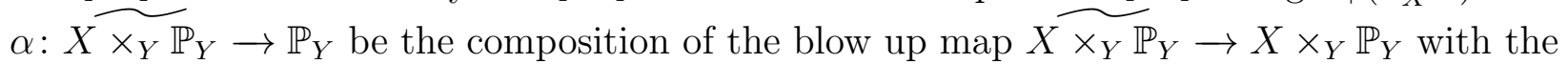
imbedding $X \times_{Y} \mathbb{P}_{Y} \rightarrow \mathbb{P}_{Y}$. 
Let

$$
(d f)^{*}: X \times_{Y} T^{*} Y \rightarrow T^{*} X
$$

be the map adjoint to the differential $d f$. It is easy to see that it induces a smooth map to the oriented projectivization of $T^{*} X$

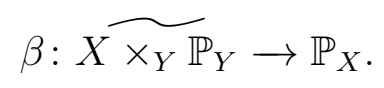

Thus consider the diagram

$$
\mathbb{P}_{X} \stackrel{\beta}{\leftarrow} \widetilde{\times_{Y} \mathbb{P}_{Y}} \stackrel{\alpha}{\rightarrow} \mathbb{P}_{Y} .
$$

3.1.2 Proposition. Let $\xi \in V^{\infty}(Y)$ be a smooth valuation given by $\xi(P)=\int_{P} \mu+\int_{N(P)} \omega$ where $\mu \in \Omega^{m}(Y), \omega \in \Omega^{m-1}(Y)$. Then $f^{*} \xi$ is given by $\left(f^{*} \xi\right)(P)=\int_{P} \mu^{\prime}+\int_{N(P)} \omega^{\prime}$ where

$$
\mu^{\prime}=q_{*} \theta^{*} \omega, \omega^{\prime}=\beta_{*} \alpha^{*} \omega .
$$

Proof. It follows immediately from the definition of $f^{*}$. Q.E.D.

One can easily deduce from Proposition 3.1.2 the following result that pull-back preserves the filtration $\left\{W_{\bullet}\right\}$ on valuations discussed in Section 1.6,

3.1.3 Proposition. If $f: X \rightarrow Y$ is a smooth immersion then for any $i$ one has on smooth valuations

$$
f^{*}\left(W_{i}\right) \subset W_{i}
$$

\subsection{Push-forward of smooth valuations under submersions.}

Let

$$
f: X^{n} \rightarrow Y^{m}
$$

be a smooth proper submersion. We define an operator of push-forward

$$
f_{*}: V^{\infty}(X) \rightarrow V^{\infty}(Y)
$$

by the formula

$$
\left(f_{*} \phi\right)(P)=\phi\left(f^{-1}(P)\right)
$$

for any $P \in \mathcal{P}(Y)$. The following claim follows from Proposition 3.2 .3 below.

3.2.1 Claim. If $f$ is a proper submersion then $f_{*}: V^{\infty}(X) \rightarrow V^{\infty}(Y)$ is a continuous linear operator.

3.2.2 Remark. If $f: X \rightarrow Y$ is a submersion which is not necessarily proper, then similarly the formula (3.2.1) defines a continuous linear operator

$$
f_{*}: V_{c}^{\infty}(X) \rightarrow V_{c}^{\infty}(Y)
$$

when both spaces are equipped with topologies of inductive limits of Fréchet spaces. This also follows from Proposition 3.2 .3 below. 
Let us describe the operation of push-forward on the language of differential forms. Let $\phi \in V_{c}^{\infty}(X)$. By [7], Lemma 2.1.1, there exist $(\nu, \omega) \in \Omega_{c}^{n}(X) \times \Omega_{c}^{n-1}\left(\mathbb{P}_{X}\right)$ such that $\phi(P)=$ $\int_{P} \nu+\int_{N(P)} \omega$ for any $P \in \mathcal{P}(X)$. (In the case when $f$ is proper, it is not necessary to consider valuations with compact support.)

Let us consider the following diagram

$$
\mathbb{P}_{X} \stackrel{(d f)^{*}}{\hookleftarrow} X \times_{Y} \mathbb{P}_{Y} \stackrel{p}{\rightarrow} \mathbb{P}_{Y}
$$

where $p$ is the natural projection, and $(d f)^{*}$ is the adjoint of the differential $d f$. Clearly $(d f)^{*}$ is a closed imbedding since $f$ is a submersion.

3.2.3 Proposition. The valuation $f_{*} \phi$ is represented by the pair $\left(f_{*} \nu, p_{*}\left(d f^{*}\right)^{*} \omega\right)$.

Proof. It is straightforward and left to a reader. Q.E.D.

One can easily see from the definitions that $f_{*}$ respects the filtration in the following way.

3.2.4 Proposition. Let $f: X \rightarrow Y$ is a proper submersion. Then for any $i$ one has on smooth valuations

$$
f_{*}\left(W_{i}\right) \subset W_{i-\operatorname{dim} X+\operatorname{dim} Y}
$$

where one defines $W_{j}=W_{0}$ for $j<0$.

\subsection{Pull-back of generalized valuations under submersions.}

Let

$$
f: X^{n} \rightarrow Y^{m}
$$

be a submersion of smooth manifolds. Let us define a pull-back operator

$$
f^{*}: V^{-\infty}(Y) \rightarrow V^{-\infty}(X)
$$

as the adjoint operator to $f_{*}: V_{c}^{\infty}(X) \rightarrow V_{c}^{\infty}(Y)$ defined in Section 3.2 .

3.3.1 Remark. Under submersions, pull-back of smooth valuations may be not smooth. For example let us consider the projection $f: \mathbb{R}^{2} \rightarrow \mathbb{R}$ onto the first coordinate. Let $\mu$ be a Lebesgue measure on $\mathbb{R}$ (which is a smooth valuation of course). Then $f^{*} \mu$ is not smooth. In fact $f^{*} \mu$ can be given the following description which is not completely rigorous at this point. For any convex compact set $K \subset \mathbb{R}^{2}$ one has

$$
\left(f^{*} \mu\right)(K)=\mu(f(K))
$$

is the Lebesgue measure of the projection of $K$.

3.3.2 Example. The example from the previous remark can be generalized as follows. Again, we do not make here the statements completely rigorous in order to clarify the intuition. Let $f: \mathbb{R}^{n} \rightarrow \mathbb{R}^{k}$ be an orthogonal projection. Let $\phi$ be a smooth valuation on $\mathbb{R}^{k}$, for example volume $v l_{k}$ or intrinsic volume $V_{i}$. Then for any convex compact set $K \subset \mathbb{R}^{n}$ one has

$$
\left(f^{*} \phi\right)(K)=\phi(f(K))
$$


Moreover if $P \in \mathcal{P}\left(\mathbb{R}^{n}\right)$ is a compact submanifold with corners which is "in generic position" to the projection $f$, then

$$
\left(f^{*} \operatorname{vol}_{k}\right)(P)=\int_{y \in \mathbb{R}^{k}} \chi\left(P \cap f^{-1}(y)\right) \operatorname{dvol}_{k}(y) .
$$

Let us describe the pull-back on valuations in terms of currents. As in Section 3.2 , let us consider the following diagram

$$
\mathbb{P}_{X} \stackrel{d f^{*}}{\hookleftarrow} X \times_{Y} \mathbb{P}_{Y} \stackrel{p}{\rightarrow} \mathbb{P}_{Y}
$$

where $p$ is the natural projection, and $d f^{*}$ is the adjoint of the differential $d f$. We have the following result.

3.3.3 Proposition. Let $f: X^{n} \rightarrow Y^{m}$ be a smooth submersion. Let $\xi \in V^{-\infty}(Y)$ corresponds to a pair of currents $(C, T) \in \mathcal{D}_{m}(Y) \times \mathcal{D}_{m-1}\left(\mathbb{P}_{Y}\right)$. Then $f^{*} \xi \in V^{-\infty}(X)$ corresponds to the pair of currents $\left(C^{\prime}, T^{\prime}\right) \in \mathcal{D}_{n}(X) \times \mathcal{D}_{n-1}\left(\mathbb{P}_{X}\right)$ where

$$
\begin{array}{r}
C^{\prime}=f^{*} C, \\
T^{\prime}=\left(d f^{*}\right)_{*} p^{*} T .
\end{array}
$$

Proof. Let $\phi \in V_{c}^{\infty}(X)$ be an arbitrary smooth valuation with compact support. By [7], Lemma 2.1.1, there exist $(\nu, \omega) \in \Omega_{c}^{n}(X) \times \Omega_{c}^{n-1}\left(\mathbb{P}_{X}\right)$ such that $\phi(P)=\int_{P} \nu+\int_{N(P)} \omega$ for any $P \in \mathcal{P}(X)$. By Proposition 3.2 .3

$$
\begin{aligned}
<\xi, f_{*} \phi>=< & C, f_{*} \nu>+<T, p_{*}\left(d f^{*}\right)^{*} \omega>= \\
& <f^{*} C, \nu>+<\left(d f^{*}\right)_{*} p^{*} T, \omega>.
\end{aligned}
$$

The proposition is proved. Q.E.D.

3.3.4 Proposition. Let $f: X \rightarrow Y$ be a submersion. Let $P \in \mathcal{P}(Y)$. Then $f^{*}\left(\Xi_{\mathcal{P}}\left(\mathbb{1}_{P}\right)\right)=$ $\Xi_{\mathcal{P}}\left(\mathbb{1}_{f^{-1}(P)}\right)$. In other words the pull-back of $\mathbb{1}_{P}$ considered as a generalized valuation coincides with the usual pull-back on (constructible) functions.

Proof. This follows from Proposition 3.3.3, Q.E.D.

By dualizing Proposition 3.2.4 one easily sees that pull-back is compatible with filtrations, namely we have the following result.

3.3.5 Proposition. Let $f: X \rightarrow Y$ be a submersion. Then on generalized valuations one has

$$
f^{*}\left(W_{i}\left(V^{-\infty}(Y)\right)\right) \subset W_{i}\left(V^{-\infty}(X)\right)
$$

\subsection{Push-forward of generalized valuations under immersions.}

Let

$$
f: X \rightarrow Y
$$

be a smooth immersion. Let us define the push-forward operator

$$
f_{*}: V_{c}^{-\infty}(X) \rightarrow V_{c}^{-\infty}(Y)
$$


as the adjoint operator to pull-back $f^{*}: V^{\infty}(Y) \rightarrow V^{\infty}(X)$ defined in Section 3.1. Similarly for a proper immersion $f$ we define $f_{*}: V^{-\infty}(X) \rightarrow V^{-\infty}(Y)$ as dual to $f^{*}: V_{c}^{\infty}(Y) \rightarrow$ $V_{c}^{\infty}(X)$.

3.4.1 Remark. (1) The push-forward under immersion of a smooth valuation is usually not smooth. For example consider the imbedding $f:\{0\} \hookrightarrow \mathbb{R}$. The $f_{*}(1)$ is the delta-measure at 0 .

(2) The space of generalized densities (which is the completion of the space of smooth measures in the weak topology) is a subspace of generalized valuations by [7, Proposition 7.3.5. Then the push-forward under immersions (and, in fact, under more general maps) on this subspace coincides with the usual push-forward of generalized densities.

(3) If $f: X \rightarrow Y$ is a closed imbedding and $P \in \mathcal{P}(X)$, then $f_{*}\left(\Xi_{\mathcal{P}}\left(\mathbb{1}_{P}\right)\right)=\Xi_{\mathcal{P}}\left(\mathbb{1}_{P}\right)$. Thus push-forward under a closed imbedding of constructible functions is just extension by zero.

Let us describe now the push-forward $f_{*}$ using the language of currents. We will assume for simplicity that $f: X \rightarrow Y$ is a closed imbedding, and $\operatorname{dim} X<\operatorname{dim} Y$ (of course, if $\operatorname{dim} X=\operatorname{dim} Y$ the description is evident). By our definition, $f_{*}: V^{-\infty}(X) \rightarrow V^{-\infty}(Y)$ is dual to $f^{*}: V_{c}^{\infty}(Y) \rightarrow V_{c}^{\infty}(X)$.

Consider the diagram as in (3.1.3)

$$
\mathbb{P}_{X} \stackrel{\beta}{\leftarrow \times \times_{Y} \mathbb{P}_{Y}} \stackrel{\alpha}{\rightarrow} \mathbb{P}_{Y}
$$

where $\alpha=\delta \circ \varepsilon$ with

$$
\widetilde{X \times_{Y} \mathbb{P}_{Y}} \stackrel{\varepsilon}{\rightarrow} X \times_{Y} \mathbb{P}_{Y} \stackrel{\delta}{\rightarrow} \mathbb{P}_{Y}
$$

with $\varepsilon$ being the oriented blow up map, $\delta$ being the natural imbedding, and $\beta$ is induced by the dual of the differential $d f^{*}: X \times_{Y} T^{*} Y \rightarrow T^{*} X$.

3.4.2 Proposition. Assume that $\phi \in V^{-\infty}(X)$ is given by a pair of currents $(C, T)$. Then $f_{*} \phi$ is given by the pair $\left(0, \alpha_{*} \beta^{*} T\right)$.

Proof. Notice first of all that the current $\alpha_{*} \beta^{*} T$ is well defined since $\beta$ is a submersion. The operator $(C, T) \mapsto \alpha_{*} \beta^{*} T$ is a continuous linear operator

$$
V^{-\infty}(X) \rightarrow \mathcal{D}_{\operatorname{dim} Y-1}\left(\mathbb{P}_{Y}\right)
$$

Hence it is enough to prove the proposition for a dense subspace of generalized valuations. Thus it is sufficient to prove it for $\phi=\Xi_{\mathcal{P}}\left(\mathbb{1}_{P}\right)$ for any $P \in \mathcal{P}(X)$. By Remark 3.4.1)(3) $f_{*}\left(\Xi_{\mathcal{P}}\left(\mathbb{1}_{P}\right)\right)=\Xi_{\mathcal{P}}\left(\mathbb{1}_{P}\right)$. But the normal cycle of $P$ as a subset of $Y$ is equal to $\alpha_{*} \beta^{*}$ of the normal cycle of $P$ considered as a subset of $X$. Q.E.D.

Dualizing Proposition 3.1.3 one easily obtains the following proposition.

3.4.3 Proposition. Let $f: X \rightarrow Y$ be a closed imbedding. Then for any $i$ one has on generalized valuations

$$
f_{*}\left(W_{i}\left(V^{-\infty}(X)\right)\right) \subset W_{i-\operatorname{dim} X+\operatorname{dim} Y}\left(V^{-\infty}(Y)\right)
$$




\subsection{Pull-back of generalized valuations under immersions.}

This case is somewhat more involved than the previous ones. Let

$$
f: X^{n} \rightarrow Y^{m}
$$

be an immersion. We want to construct a partially defined map $f^{*}: V^{-\infty}(Y) \rightarrow V^{-\infty}(X)$ which coincides on smooth valuations with the map from Section 3.1.

Generalized valuations form a sheaf by [7], Proposition 7.2.2. Thus it is sufficient to define $f^{*}$ locally in a way compatible with restrictions to open subsets. Hence we may assume that $f: X \rightarrow Y$ is a closed imbedding.

Consider the diagram as in (3.1.3)

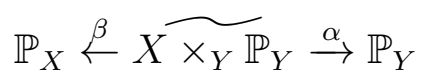

where $\alpha=\delta \circ \varepsilon$ with

$$
\widetilde{X \times_{Y} \mathbb{P}_{Y}} \stackrel{\varepsilon}{\rightarrow} X \times_{Y} \mathbb{P}_{Y} \stackrel{\delta}{\rightarrow} \mathbb{P}_{Y}
$$

with $\varepsilon$ being the oriented blow up map, $\delta$ being the natural imbedding.

Let $\xi \in V^{-\infty}(Y)$ be a generalized valuation given by a pair of currents $(C, T) \in \mathcal{D}_{m}(Y) \times$ $\mathcal{D}_{m-1}\left(\mathbb{P}_{Y}\right)$. Then we define $f^{*} \xi$ to correspond to the pair

$$
\left(C^{\prime}, T^{\prime}\right):=\left(f^{*} C, \beta_{*} \alpha^{*} T\right)
$$

whenever this makes sense. Now we are going to formulate the precise sufficient conditions that the above pair is well defined, and to show that $T^{\prime}$ is Legendrian, $\partial T^{\prime}=0$, and $\pi_{X *} T^{\prime}=$ $\partial C^{\prime}$, and prove various compatibilities.

Let us introduce more notation. Let

$$
\begin{aligned}
& \Lambda \subset T^{*} Y \backslash \underline{0}, \\
& \Gamma \subset T^{*} \mathbb{P}_{Y} \backslash \underline{0}
\end{aligned}
$$

be closed conic subsets. Let us denote by $V_{\Lambda, \Gamma}^{-\infty}(Y)$ the linear subspace of generalized valuations on $Y$ corresponding to currents $(C, T)$ with $W F(C) \subset \Lambda, W F(T) \subset \Gamma$. Thus

$$
V_{\Lambda, \Gamma}^{-\infty}(Y) \subset C_{\Lambda}^{-\infty}(Y) \times C_{\Gamma}^{-\infty}\left(\mathbb{P}_{Y}, \Omega^{\operatorname{dim} Y}\right)
$$

is a closed subspace. Let us equip $V_{\Lambda, \Gamma}^{-\infty}(Y)$ with the induced topology from the latter space (see Section 1.4).

3.5.1 Definition. (1) We say that an imbedding $f: X \rightarrow Y$ is transversal to $(\Lambda, \Gamma)$ if the following conditions of transversality are satisfied:

$\left(T R_{1}\right) \Lambda \cap T_{X}^{*} Y=\emptyset$;

$\left(T R_{2}\right) \Gamma \cap T_{X \times_{Y} \mathbb{P}_{Y}}^{*}\left(\mathbb{P}_{Y}\right)=\emptyset$

$\left(T R_{3}\right) \Gamma \cap T_{\mathbb{P}_{+}\left(T_{X}^{*} Y\right)}^{*}\left(\mathbb{P}_{Y}\right)=\emptyset$.

(2) We say that an immersion $f: X \rightarrow Y$ is transversal to $(\Lambda, \Gamma)$ if for every point $x \in X$ there exist an open neighborhood $U$ of $x$ and an open neighborhood $V$ of $f(x)$ such that $\left.f\right|_{U}: U \rightarrow V$ is a closed imbedding, and $\left.f\right|_{U}$ is transversal to $(\Lambda, \Gamma)$ in the sense of part (1) of the definition. 
3.5.2 Definition. Let $f: X \rightarrow Y$ be an immersion. Let $\xi \in V^{-\infty}(Y)$ be a generalized valuation given by a pair $(C, T)$. We say that $f$ is transversal to $\xi$ if $f$ is transversal to $(W F(C), W F(T))$ in the sense of Definition 3.5.1(2).

3.5.3 Remark. (1) It is easy to see that if $f$ is a smooth imbedding then parts (1) and (2) of Definition 3.5 .1 are equivalent.

(2) If $\xi$ is a smooth valuation then any immersion $f: X \rightarrow Y$ is transversal to $\xi$ for trivial reasons.

3.5.4 Claim. Let $f: X \rightarrow Y$ be an immersion transversal to a generalized valuation $\xi \in$ $V^{-\infty}(Y)$. Then the currents (3.5.3) are well defined, and $f^{*}: V_{\Lambda, \Gamma}^{-\infty}(Y) \rightarrow V^{-\infty}(X)$ is a sequentially continuous linear map where $(\Lambda, \Gamma):=(W F(C), W F(T))$.

Proof. This easily follows from Propositions 1.4.4, 1.4.10, Q.E.D.

3.5.5 Proposition. If $f: X \rightarrow Y$ is an immersion, and $\xi \in V^{\infty}(Y)$ is a smooth valuation then the pull-back of $\xi$ in the sense of generalized valuations and in the sense of smooth valuations (Section 3.1) coincide.

Proof. Recall that we have diagrams of maps

$$
\begin{aligned}
& X \stackrel{q}{\leftarrow} \mathbb{P}_{+}\left(T_{X}^{*} Y\right) \stackrel{\theta}{\hookrightarrow} \mathbb{P}_{Y}, \\
& \mathbb{P}_{X} \stackrel{\beta}{\leftarrow} \widetilde{\times_{Y} \mathbb{P}_{Y}} \stackrel{\alpha}{\rightarrow} \mathbb{P}_{Y} \text {. }
\end{aligned}
$$

Let $\xi \in V^{\infty}(Y)$ be given by

$$
\xi(P)=\int_{P} \mu+\int_{N(P)} \omega .
$$

Then by [13] (see also Theorem 1.6.5 of this article) $\xi$ corresponds to a pair of currents $(C, T$ ) where

$$
C=\pi_{Y *} \omega, T=s_{Y}^{*}\left(D_{Y} \omega+\pi_{Y}^{*} \mu\right),
$$

and $D_{Y}$ is the Rumin operator on $\mathbb{P}_{Y}$. Then the pull-back $f^{*} \xi$ in the sense of generalized valuations is given by the pair of currents $\left(C^{\prime}, T^{\prime}\right)$ where

$$
C^{\prime}=f^{*}\left(\pi_{Y *} \omega\right), T^{\prime}=\beta_{*} \alpha^{*} s_{Y}^{*}\left(D_{Y} \omega+\pi_{Y}^{*} \mu\right)
$$

where $\alpha, \beta$ are as in (3.1.3).

On the other hand let us compute $f^{*} \xi$ in the smooth sense. By Proposition $3.1 .2 f^{*} \xi$ is given by $\left(f^{*} \xi\right)(P)=\int_{P} \mu^{\prime}+\int_{N(P)} \omega^{\prime}$ where

$$
\mu^{\prime}=q_{*} \theta^{*} \omega, \omega^{\prime}=\beta_{*} \alpha^{*} \omega .
$$

Thus we have to show that

$$
\begin{array}{r}
C^{\prime}=\pi_{X *}\left(\beta_{*} \alpha^{*} \omega\right), \\
T^{\prime}=s_{X}^{*}\left(D_{X}\left(\beta_{*} \alpha^{*} \omega\right)+\pi_{X}^{*} q_{*} \theta^{*} \omega\right) .
\end{array}
$$


More explicitly

$$
\begin{array}{r}
f^{*}\left(\pi_{Y *} \omega\right)=\pi_{X *}\left(\beta_{*} \alpha^{*} \omega\right), \\
\beta_{*} \alpha^{*} s_{Y}^{*}\left(D_{Y} \omega+\pi_{Y}^{*} \mu\right)=s_{X}^{*}\left(D_{X}\left(\beta_{*} \alpha^{*} \omega\right)+\pi_{X}^{*} q_{*} \theta^{*} \omega\right) .
\end{array}
$$

The first equality is straightforward. To prove the second one let us observe first of all that

$$
\alpha^{*} s_{Y}^{*} \pi_{Y}^{*} \mu=\alpha^{*} \pi_{Y}^{*} \mu=0
$$

Thus it remains to show that for any $\omega \in \Omega^{m-1}(Y)$ one has

$$
\beta_{*} \alpha^{*} s_{Y}^{*} D_{Y} \omega=s_{X}^{*}\left(D_{X}\left(\beta_{*} \alpha^{*} \omega\right)+\pi_{X}^{*} q_{*} \theta^{*} \omega\right) .
$$

Since $\alpha$ commutes with $s_{Y}$, and $\beta$ intertwines $s_{Y}$ and $s_{X}$, the last equality is equivalent to

$$
\beta_{*} \alpha^{*} D_{Y} \omega=D_{X}\left(\beta_{*} \alpha^{*} \omega\right)+\pi_{X}^{*} q_{*} \theta^{*} \omega
$$

We will need the following well known lemma which follows easily from the Stokes formula.

3.5.6 Lemma. Let $f: A \rightarrow B$ be a smooth proper fibration where $B$ is a manifold without boundary, and $A$ is a manifold possibly with boundary. Assume moreover that the restriction

$$
\left.f\right|_{\partial A}: \partial A \rightarrow B
$$

is a fibration too. Then

$$
f_{*}(d \eta)=d\left(f_{*} \eta\right)+(-1)^{\operatorname{dim} A+\operatorname{deg} \eta+1}\left(\left.f\right|_{\partial A}\right)_{*} \eta .
$$

Let us apply this lemma to the form $\alpha^{*} \omega$ and the map

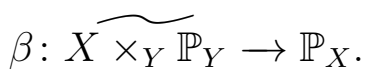

We get

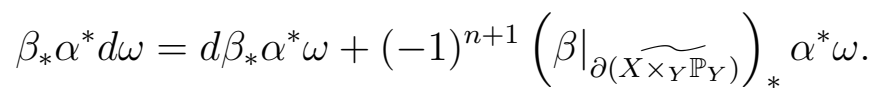

We will need two more lemmas whose proofs are postponed till the end of the proof of Proposition 3.5.5.

3.5.7 Lemma. Let $\omega \in \Omega^{\bullet}\left(\mathbb{P}_{Y}\right)$. If $d \omega$ is vertical then $d \beta_{*} \alpha^{*} \omega$ is also vertical.

3.5.8 Lemma. For any form $\omega \in \Omega^{m-1}\left(\mathbb{P}_{Y}\right)$ one has

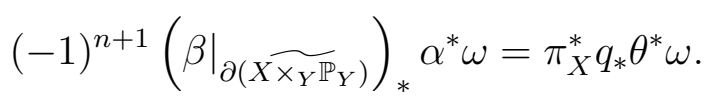

Let us finish the proof of Proposition 3.5.5. The equality (3.5.5) and Lemma 3.5.8 imply the equality

$$
\beta_{*} \alpha^{*} d \omega=d \beta_{*} \alpha^{*} \omega+\pi_{X}^{*} q_{*} \theta^{*} \omega
$$


Observe first of all that if $\gamma \in \Omega^{\bullet}\left(\mathbb{P}_{Y}\right)$ is a vertical form then $\theta^{*} \gamma=0$ since $\mathbb{P}_{+}\left(T_{X}^{*} Y\right)=N(X)$ is a Legendrian cycle in $\mathbb{P}_{Y}$. Hence, by the construction of the Rumin operator, one may assume in equality (3.5.6) that $d \omega$ is vertical, namely $d \omega=D_{Y} \omega$. But then by Lemma 3.5.7 we have $d \beta_{*} \alpha^{*} \omega=D_{X} \beta_{*} \alpha^{*} \omega$. Thus we eventually obtain

$$
\beta_{*} \alpha^{*} D_{Y} \omega=D_{X} \beta_{*} \alpha^{*} \omega+\pi_{X}^{*} q_{*} \theta^{*} \omega .
$$

This is exactly (3.5.4). Proposition 3.5.5 is proved. Q.E.D.

Proof of Lemma 3.5.7. Let $\lambda_{X}$ be a contact form on $X$, and $\lambda_{Y}$ be a contact form on $Y$. Then we claim that

$$
\beta^{*}\left(\lambda_{X}\right)=h \cdot \alpha^{*}\left(\lambda_{Y}\right)
$$

where $h$ is a smooth non-vanishing function on $\widetilde{X \times_{Y} \mathbb{P}_{Y}}$. Let us prove it in local coordinates. Let us assume that $X=\mathbb{R}^{n}$ is imbedded into $Y=\mathbb{R}^{m}$ using the first $n$ coordinates. Let us denote the coordinates in $Y$ by $y_{1}, \ldots, y_{m}$, and in the dual space by $z_{1}, \ldots, z_{m}$. Furthermore we will identify $\mathbb{P}_{Y}$ with the unit sphere bundle in $\mathbb{R}^{m} \times S^{m-1} \subset \mathbb{R}^{m} \times \mathbb{R}^{m *}$, and $\mathbb{P}_{X}$ with the unit sphere bundle $\mathbb{R}^{n} \times S^{n-1}$. Contact form on a contact manifold is defined up to a product by a non-vanishing function. Then we may choose

$$
\lambda_{Y}=\sum_{i=1}^{m} z_{i} d y_{i}, \lambda_{X}=\sum_{i=1}^{n} z_{i} d y_{i} .
$$

It is clear that the restriction of $\lambda_{Y}$ to $X \times_{Y} T^{*} Y=\mathbb{R}^{n} \times \mathbb{R}^{m *}$ is equal to $\sum_{i=1}^{n} z_{i} d y_{i}$. But obviously this is equal to the pull-back of $\lambda_{X}$ under the natural map $X \times_{Y} T^{*} Y \rightarrow T^{*} X$. This proves (3.5.7).

Next by the assumption of the lemma we have $d \omega \wedge \lambda_{Y}=0$. We want to show that

$$
d\left(\beta_{*} \alpha^{*} \omega\right) \wedge \lambda_{X}=0 .
$$

We have for any test form $\phi \in \Omega_{c}^{\bullet}\left(\mathbb{P}_{X}\right)$

$$
\begin{aligned}
& \int_{\mathbb{P}_{X}} \phi \wedge \lambda_{X} \wedge d\left(\beta_{*} \alpha^{*} \omega\right) \stackrel{3.5 .5}{=} \int_{\mathbb{P}_{X}} \phi \wedge \lambda_{X} \wedge\left(\beta_{*} \alpha^{*} d \omega+(-1)^{n}\left(\left.\beta\right|_{\partial\left(X \times_{Y} \mathbb{P}_{Y}\right)}\right)_{*} \alpha^{*} \omega\right)= \\
& \int_{X \times_{Y} \mathbb{P}_{Y}} \beta^{*}\left(\phi \wedge \lambda_{X}\right) \wedge \alpha^{*}(d \omega)+(-1)^{n} \int_{\partial\left(X \times_{Y} \mathbb{P}_{Y}\right)}\left(\left.\beta\right|_{X \times_{Y} \mathbb{P}_{Y}}\right)^{*}\left(\phi \wedge \lambda_{X}\right) \wedge \alpha^{*} \omega \stackrel{\text { 3.5.7 }}{=} \\
& \int_{X \widetilde{\times_{Y} \mathbb{P}_{Y}}} h \cdot \beta^{*} \phi \wedge \alpha^{*}\left(\lambda_{Y} \wedge d \omega\right)+(-1)^{n} \int_{\partial\left(\widetilde{\left.X \times_{Y} \mathbb{P}_{Y}\right)}\right.} h \cdot \beta^{*} \phi \wedge \alpha^{*}\left(\lambda_{Y} \wedge \omega\right)= \\
& (-1)^{n} \int_{\partial\left(X \times_{Y} \mathbb{P}_{Y}\right)} h \cdot \beta^{*} \phi \wedge \alpha^{*}\left(\lambda_{Y} \wedge \omega\right)
\end{aligned}
$$

where the last equality is due to the fact that the expression under the first integral vanishes by the assumption $\lambda_{Y} \wedge d \omega=0$. But the expression under the last integral also vanishes since the restriction of $\alpha^{*} \lambda_{Y}$ to $\partial\left(\widetilde{X \times_{Y} \mathbb{P}_{Y}}\right)$ vanishes: indeed $\partial\left(\widetilde{X \times_{Y} \mathbb{P}_{Y}}\right)$ is the primage of 
$\mathbb{P}_{+}\left(T_{X}^{*} Y\right)=N(X)$ under the blow up map $\widetilde{X \times_{Y} \mathbb{P}_{Y}} \rightarrow X \times_{Y} \mathbb{P}_{Y}$, and the restriction of $\lambda_{Y}$ to $N(X)$ vanishes since $N(X)$ is Legendrian. Hence Lemma 3.5.7 is proved. Q.E.D.

Proof of Lemma 3.5.8. Consider the following commutative diagram

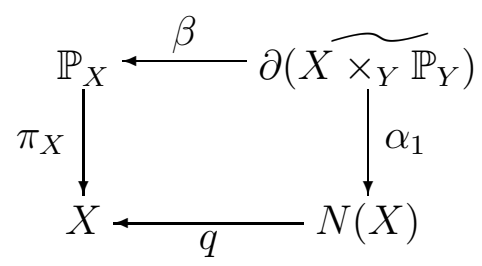

where $\alpha_{1}: \partial\left(\widetilde{X \times_{Y} \mathbb{P}_{Y}}\right) \rightarrow N(X)$ is the restriction of the blow up map. It is easy to see that this is a Cartesian square. Denote $\tau:=\theta^{*} \omega=\left.\omega\right|_{N(X)}$. Thus we have to show that

$$
(-1)^{n+1}\left(\left.\beta\right|_{\partial\left(X \times_{Y} \mathbb{P}_{Y}\right)}\right)_{*} \alpha_{1}^{*} \tau=\pi_{X}^{*} q_{*} \tau
$$

Taking into account the orientations, the last formula holds always for all Cartesian squares of proper fibrations (the base change formula). Q.E.D.

3.5.9 Proposition. Let $f: X \rightarrow Y$ be an immersion transversal to $\xi \in V^{-\infty}(Y)$. Let $\xi$ be given by a pair of currents $(C, T) \in \mathcal{D}_{m}(Y) \times \mathcal{D}_{m-1}\left(\mathbb{P}_{Y}\right)$. Define $\left(C^{\prime}, T^{\prime}\right) \in \mathcal{D}_{n}(X) \times \mathcal{D}_{n-1}\left(\mathbb{P}_{X}\right)$ by the formula (3.5.3). Then $T^{\prime}$ is Legendrian, $\partial T^{\prime}=0$, and $\pi_{X *}\left(T^{\prime}\right)=\partial C^{\prime}$.

Proof. Let us denote

$$
\Lambda:=W F(C), \Gamma:=W F(T) .
$$

The formula (3.5.3) defines a sequentially continuous linear operator

$$
f^{*}: C_{\Lambda}^{-\infty}(Y) \oplus C_{\Gamma}^{-\infty}\left(\mathbb{P}_{Y}, \Omega^{m}\right) \rightarrow C^{-\infty}(X) \oplus C^{-\infty}\left(\mathbb{P}_{X}, \Omega^{n}\right)
$$

which we also denote by $f^{*}$. By Lemma 1.6.8 locally on $Y$ there exists a sequence of smooth valuations $\left\{\xi_{j}\right\} \subset V^{\infty}(Y)$ such that

$$
\xi_{j} \rightarrow \xi \text { in } V_{\Lambda, \Gamma}^{-\infty}(Y)
$$

But then from the properties of wave fronts we have

$$
f^{*} \xi_{j} \rightarrow f^{*} \xi \text { in } V^{-\infty}(X)
$$

Let $\xi_{j}$ correspond to a pair of smooth currents $\left(C_{j}, T_{j}\right)$. By Proposition 3.5.5 the conclusion of our proposition is satisfied for smooth currents $f^{*}\left(\left(C_{j}, T_{j}\right)\right)$ corresponding to $f^{*} \xi_{j}$. Hence by sequential continuity the proposition is satisfied also by $\left(C^{\prime}, T^{\prime}\right)$. Q.E.D.

3.5.10 Remark. It is important to notice that if $\Lambda \subset T^{*} Y \backslash \underline{0}, \Gamma \subset T^{*}\left(\mathbb{P}_{Y}\right) \backslash \underline{0}$ are closed conic subsets, and if an imbedding $f: X \rightarrow Y$ is transversal to $(\Lambda, \Gamma)$ then the pull-back

$$
f^{*}: V_{\Lambda, \Gamma}^{-\infty}(Y) \rightarrow V_{f^{*} \Lambda, \beta_{*} \alpha^{*} \Gamma}^{-\infty}(X)
$$

is a sequentially continuous linear operator. This is obvious from the properties of wave fronts. 
3.5.11 Proposition. Let $g: X \rightarrow Y, f: Y \rightarrow Z$ be imbeddings. Let $\xi \in V_{\Lambda, \Gamma}^{-\infty}(Z)$. Assume that $f$ and $f \circ g$ are transversal to $(\Lambda, \Gamma)$, and $g$ is transversal to $\left(f^{*} \Lambda, \beta_{*} \alpha^{*} \Gamma\right)$. Then the maps

$$
(f \circ g)^{*}, g^{*} \circ f^{*}: V_{\Lambda, \Gamma}^{-\infty}(Z) \rightarrow V^{-\infty}(X)
$$

coincide.

Proof. Since the statement is local, we may assume that $X, Y, Z$ are vector spaces. The assumptions imply that the two linear operators

$$
(f \circ g)^{*}, g^{*} \circ f^{*}: V_{\Lambda, \Gamma}^{-\infty}(Z) \rightarrow V^{-\infty}(X)
$$

are sequentially continuous. By Lemma 1.6.8 smooth valuations are sequentially dense in the source space. Hence it is enough to show that these two operators coincide on smooth valuations. But this is obvious. Q.E.D.

3.5.12 Proposition. Let $f: X \rightarrow Y$ be a closed imbedding. Let $P \in \mathcal{P}(Y)$ be a compact submanifold with corners such that $X$ is transversal to any strata of the canonical subdivision of $P$. Then $f$ is transversal to the generalized valuation $\Xi_{\mathcal{P}}\left(\mathbb{1}_{P}\right)$ and

$$
f^{*}\left(\Xi_{\mathcal{P}}\left(\mathbb{1}_{P}\right)\right)=\Xi_{\mathcal{P}}\left(\mathbb{1}_{P \cap X}\right)
$$

Proof. Let us denote $\Lambda:=W F\left(\mathbb{1}_{P}\right), \Gamma:=W F([[N(P)]])$. First we have to check the conditions of transversality $\left(T R_{1}\right)-\left(T R_{3}\right)$ from Definition 3.5.1,

The condition $\left(T R_{1}\right)$ easily follows from the transversality of $X$ to every stratum of $P$ and the observation that $\Lambda$ is contained in the union of conormal bundles to all strata of $P$.

The condition $\left(T R_{3}\right)$ immediately follows from the observation that

$$
N(P) \cap \mathbb{P}_{+}\left(T_{X}^{*} Y\right)=\emptyset
$$

which again is a simple consequence of transversality of $X$ to all the strata of $P$.

Let us check $\left(T R_{2}\right)$. This condition is equivalent to

$$
W F([[N(P)]]) \cap \pi_{Y}^{*}\left(T_{X}^{*} Y\right)=\emptyset
$$

where $\pi_{Y}: \mathbb{P}_{Y} \rightarrow Y$ is the natural projection. Let us denote $P_{0}:=P \cap X$ which is a submanifold with corners of $X$ (again due to the transversality of $X$ and strata of $P$ ). Let us fix a point $x_{0} \in P_{0}$. Again as a consequence of transversality of $X$ and strata of $P$, in a neighborhood of $x_{0}$ we can write $Y=X \times Z, P=P_{0} \times Z$ where $X$ and $Z$ may and will be assumed vector spaces (for the convenience of the notation), $P_{0} \subset X$. Then one has

$$
N(P)=\left\{\left(x, z ;\left[\xi: 0_{Z^{*}}\right]\right) \mid(x,[\xi]) \in N\left(P_{0}\right), z \in Z\right\} \subset \mathbb{P}_{Y}
$$

This description easily implies (3.5.9). Thus we have shown that $f^{*}\left(\Xi_{\mathcal{P}}\left(\mathbb{1}_{P}\right)\right)$ is well defined.

In order to prove the second part of the proposition, we notice that the equality $P=$ $P_{0} \times Z$ implies the equality

$$
f^{*}\left(\mathbb{1}_{P}\right)=\mathbb{1}_{P \cap X}
$$


(where $\mathbb{1}_{P} \in C^{-\infty}(Y), \mathbb{1}_{P \cap X} \in C^{-\infty}(X)$ ). Thus it remains to show that

$$
\beta_{*} \alpha^{*}([[N(P)]])=[[N(P \cap X)]]
$$

where as previously we have

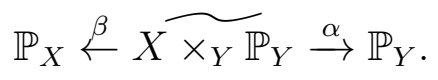

Recall that the map $\alpha$ is equal to the composition

$$
\widetilde{\times_{Y} \mathbb{P}_{Y}} \stackrel{\varepsilon}{\rightarrow} X \times_{Y} \mathbb{P}_{Y} \stackrel{\delta}{\rightarrow} \mathbb{P}_{Y}
$$

where $\delta$ is the natural imbedding, and $\varepsilon$ is the oriented blow up map along $\mathbb{P}_{+}\left(T_{X}^{*} Y\right)$.

From (3.5.10) it is easy to see that

$$
\delta^{*}([[N(P)]])=\left[\left[N(P) \cap\left(X \times_{Y} \mathbb{P}_{Y}\right)\right]\right]=\left[\left[\left\{\left(x ;\left[\xi: 0_{Z^{*}}\right]\right) \mid(x,[\xi]) \in N\left(P_{0}\right)\right\}\right]\right] .
$$

Then (3.5.12) and (3.5.8) imply readily that

$$
\beta_{*} \varepsilon^{*}\left(\delta^{*}([[N(P)]])=\left[\left[N\left(P_{0}\right)\right]\right] .\right.
$$

The equalities (3.5.11) and (3.5.13) imply the proposition. Q.E.D.

The following result will be needed later for integral geometric applications. It is another special case of the heuristic fact that $(f \circ g)^{*}=g^{*} \circ f^{*}$.

3.5.13 Proposition. Let $X \stackrel{g}{\rightarrow} Y \stackrel{f}{\rightarrow} Z$ be smooth maps such that $g$ is an immersion, $f$ is a submersion, and the composition $f \circ g: X \rightarrow Z$ is an immersion. Let $\xi \in V^{-\infty}(Z)$ be a generalized valuation. Assume that $f \circ g$ is transversal to $\xi$. Then $g$ is transversal to $f^{*} \xi$ and

$$
g^{*}\left(f^{*}(\xi)\right)=(f \circ g)^{*} \xi
$$

Proof. The proof is a straightforward tedious computation which we are going to do. First by localization we may assume that $g$ and $f \circ g$ are imbeddings. Localizing further we may assume that $X$ is a vector space, and for some vector spaces $S$ and $L$

$$
Z=X \times S, Y=X \times S \times L
$$

$$
g: X \rightarrow X \times S \times L \text { is given by } g(x)=\left(x, 0_{S}, 0_{L}\right)
$$$$
f: X \times S \times L \rightarrow X \times S \text { is the projection. }
$$

Thus $(f \circ g)(x)=\left(x, 0_{S}\right)$. Let $\xi$ be given by a pair of currents $(C, T)$. Let us denote

$$
\begin{array}{r}
\Lambda:=W F(C) \subset T^{*}(X \times S) \backslash \underline{0}, \\
\Gamma:=W F(T) \subset T^{*}\left(\mathbb{P}_{X \times S}\right) \backslash \underline{0} .
\end{array}
$$

Let us recall that we have the maps

$$
\mathbb{P}_{Y} \stackrel{d f^{*}}{\longleftarrow} \mathbb{P}_{Z} \times L \stackrel{p}{\rightarrow} \mathbb{P}_{Z}
$$


Recall that $f^{*} \xi$ is given by the pair $\left(C^{\prime}, T^{\prime}\right):=\left(f^{*} C,\left(d f^{*}\right)_{*} p^{*} T\right)$.

Step 1. Let us check that $g$ and $f^{*} \xi$ satisfy the first condition of transversality $\left(T R_{1}\right)$. More precisely let us check that $g^{*}\left(f^{*} C\right)$ is well defined, namely

$$
W F\left(f^{*} C\right) \cap T_{X}^{*}(X \times S \times L)=\emptyset .
$$

Identifying $T^{*}(X \times S \times L)=(X \times S \times L) \times\left(X^{*} \times S^{*} \times L^{*}\right)$, let us observe that

$$
T_{X}^{*}(X \times S \times L)=\left\{\left(x, 0_{S}, 0_{L} ; 0_{X^{*}}, s^{*}, l^{*}\right)\right\} .
$$

Next we have

$$
W F\left(f^{*} C\right) \subset f^{*} \Lambda=\left\{\left(x, s, l ; x^{*}, s^{*}, 0_{L^{*}}\right) \mid\left(x, s ; x^{*}, s^{*}\right) \in \Lambda\right\} .
$$

Thus

$$
f^{*} \Lambda \cap T_{X}^{*}(X \times S \times L)=\left\{\left(x, 0_{S}, 0_{L} ; 0_{X^{*}}, s^{*}, 0_{L^{*}}\right) \mid\left(x, 0_{S} ; 0_{X^{*}}, s^{*}\right) \in \Lambda\right\} .
$$

But

$$
\left\{\left(x, 0_{S} ; 0_{X^{*}}, s^{*}\right) \in \Lambda\right\}=\Lambda \cap T_{X}^{*}(X \times S)=\emptyset
$$

where the last equality follows from the assumption that $f \circ g$ is transversal to $\xi$. Thus $\left(T R_{1}\right)$ is proved.

Step 2. Let us check that $g$ and $f^{*} \xi$ satisfy the third condition of transversality $\left(T R_{3}\right)$. Namely let us check that $W F\left(T^{\prime}\right)$ does not intersect the conormal bundle of the submanifold $\mathbb{P}_{+}\left(T_{X}^{*}(X \times S \times L)\right) \subset \mathbb{P}_{X \times S \times L}$.

We have $W F\left(T^{\prime}\right)=W F\left(\left(d f^{*}\right)_{*} p^{*} T\right) \subset\left(d f^{*}\right)_{*} p^{*} \Gamma$. Let us write down the latter space more explicitly.

Let us fix an element $\left(z_{0},\left[z_{0}^{*}\right]\right)$ of the space $\mathbb{P}_{Z}=Z \times \mathbb{P}_{+}\left(Z^{*}\right)$. The fiber of $T^{*} \mathbb{P}_{Z}$ over this point is identified with $Z^{*} \times T_{\left[z_{0}^{*}\right]}^{*}\left(\mathbb{P}_{+}\left(Z^{*}\right)\right)$. Let us fix also a point $l_{0} \in L$. The fiber of $T^{*}\left(\mathbb{P}_{Z} \times L\right)$ over the point $\left(z_{0},\left[z_{0}^{*}\right], l_{0}\right)$ is equal to $Z^{*} \times T_{\left[z_{0}^{*}\right]}^{*}\left(\mathbb{P}_{+}\left(Z^{*}\right)\right) \times L^{*}$, elements of which will be denoted by $\left(z^{*}, \xi^{*}, l^{*}\right)$. In this notation we easily see that the fiber of $p^{*} \Gamma$ over the point $\left(z_{0},\left[z_{0}^{*}\right], l_{0}\right)$ is equal to

$$
\left.\left(p^{*} \Gamma\right)\right|_{\left(z_{0},\left[z_{0}^{*}, l_{0}\right)\right.}=\left\{\left(z^{*}, \xi^{*}, 0_{L^{*}}\right) \in Z^{*} \times T_{\left[z_{0}^{*}\right]}^{*}\left(\mathbb{P}_{+}\left(Z^{*}\right)\right) \times L^{*}\left|\left(z^{*}, \xi^{*}\right) \in \Gamma\right|_{\left(z_{0},\left[z_{0}^{*}\right]\right)}\right\} .
$$

Let us describe the fiber of $\tilde{\Gamma}:=\left(d f^{*}\right)_{*} p^{*} \Gamma$ over the point $\left(z_{0},\left[z_{0}^{*}: 0_{L^{*}}\right], l_{0}\right)$ (clearly the fiber of $\tilde{\Gamma}$ may be non-empty only over points of such form). Let us denote for this purpose

$$
\nu: T_{\left[z_{0}^{*}: 0_{L^{*}}\right]}^{*}\left(\mathbb{P}_{+}\left(Z^{*} \times L^{*}\right)\right) \rightarrow T_{\left[z_{0}^{*}\right]}^{*}\left(\mathbb{P}_{+}\left(Z^{*}\right)\right)
$$

the natural map, namely the dual map to the differential of the imbedding $\mathbb{P}_{+}\left(Z^{*}\right) \hookrightarrow$ $\mathbb{P}_{+}\left(Z^{*} \times L^{*}\right)$. Then using the description (3.5.14) one easily sees that

$$
\left\{\left(z^{*}, \eta^{*}, 0_{L^{*}}\right) \in Z^{*} \times T_{\left[z_{0}^{*}: 0_{L^{*}}\right]}^{*}\left(\mathbb{P}_{+}\left(Z^{*} \times L^{*}\right)\right) \times L^{*}\left|\left(z^{*}, \nu\left(\eta^{*}\right)\right) \in\{0\} \cup \Gamma\right|_{\left(z_{0},\left[z_{0}^{*}\right]\right)}\right\} \backslash\{0\} .
$$


We want to show that the intersection of $\tilde{\Gamma}$ with the conormal bundle of $\mathbb{P}_{+}\left(T_{X}^{*}(X \times S \times L)\right)$ is empty. Observe that

$$
\mathbb{P}_{+}\left(T_{X}^{*}(X \times S \times L)\right)=\left\{\left(x, 0_{S}, 0_{L} ;\left[0_{X^{*}}: s^{*}: l^{*}\right]\right)\right\}
$$

Thus we will consider the case

$$
\begin{array}{r}
z_{0}=\left(x_{0}, 0_{S}\right) \in X \times S=Z, \\
l_{0}=0_{L} \in L, \\
{\left[z_{0}^{*}\right]=\left[0_{X^{*}}: s_{0}^{*}\right] \in \mathbb{P}_{+}\left(X^{*} \times S^{*}\right) .}
\end{array}
$$

The fiber of the conormal bundle of $\mathbb{P}_{+}\left(T_{X}^{*}(X \times S \times L)\right) \subset \mathbb{P}_{Y}$ over the point $\left(z_{0}, 0_{L},\left[z_{0}^{*}: 0_{L^{*}}\right]\right)$ (which we identify with $\left(x_{0}, 0_{S}, 0_{L} ;\left[0_{X^{*}}: s_{0}^{*}: 0_{L^{*}}\right]\right)$ ) is equal to

$$
\left\{\left(0_{X^{*}}, s^{*}, l^{*} ; \xi^{*}\right) \mid \mu\left(\xi^{*}\right)=0\right\}
$$

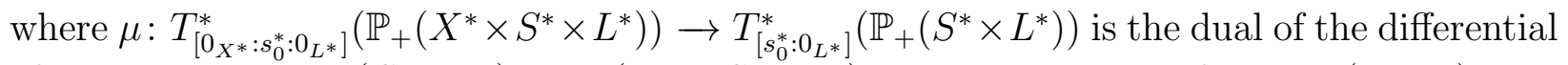
of the imbedding $\mathbb{P}_{+}\left(S^{*} \times L^{*}\right) \subset \mathbb{P}_{+}\left(X^{*} \times S^{*} \times L^{*}\right)$. The intersection of the set (3.5.15) with the fiber of $\tilde{\Gamma}$ is equal to

$$
\left\{\left(0_{X^{*}}, s^{*}, 0_{L^{*}} ; \xi^{*}\right) \mid\left(0_{X^{*}}, s^{*}, \nu\left(\xi^{*}\right)\right) \in\{0\} \cup \Gamma \text { and } \mu\left(\xi^{*}\right)=0\right\} \backslash\{0\} .
$$

It is easy to see that if $\mu\left(\xi^{*}\right)=0$ and $\xi^{*} \neq 0$, then $\nu\left(\xi^{*}\right) \neq 0$. Hence if the set (3.5.16) is non-empty and a vector $\left(0_{X^{*}}, s^{*}, 0_{L^{*}} ; \xi^{*}\right)$ belongs to it, then $\left(0_{X^{*}}, s^{*}, \nu\left(\xi^{*}\right)\right) \in \Gamma$. But then the latter vector belongs to the intersection of the fibers over $\left(x_{0}, 0_{S} ;\left[0_{X^{*}}: s_{0}\right]\right)$ of $\Gamma$ and of the conormal bundle of $\mathbb{P}_{+}\left(T_{X}^{*}(X \times S)\right) \subset \mathbb{P}_{X \times S}$ (using $\left.\mu\left(\xi^{*}\right)=0\right)$. But the last intersection is empty since the map $f \circ g$ is assumed to be transversal to $\xi$ and thus satisfies the third condition of transversality $\left(T R_{3}\right)$, namely the intersection of $\Gamma$ with the conormal bundle of $\mathbb{P}_{+}\left(T_{X}^{*}(X \times S)\right) \subset \mathbb{P}_{X \times S}$ is empty. Hence Step 2 is completed.

Step 3. Let us check that $g$ and $f^{*} \xi$ satisfy $\left(T R_{2}\right)$. Namely let us check that $W F\left(T^{\prime}\right)$ does not intersect the conormal bundle of the submanifold $X \times_{Y} \mathbb{P}_{Y} \subset \mathbb{P}_{Y}$. Recall that $Y=X \times S \times L$.

Let us fix an arbitrary point $B_{0}:=\left(x_{0}, 0_{S}, 0_{L} ;\left[x_{0}^{*}: s_{0}^{*}: l_{0}^{*}\right]\right) \in X \times_{Y} \mathbb{P}_{Y}$. We identify the fiber of $T^{*} \mathbb{P}_{Y}$ over this point with $X^{*} \times S^{*} \times L^{*} \times T_{\left[x_{0}^{*}: s_{0}^{*}: l_{0}^{*}\right]}\left(\mathbb{P}_{+}\left(X^{*} \times S^{*} \times L^{*}\right)\right)$.

The fiber of the conormal bundle of $X \times_{Y} \mathbb{P}_{Y}$ over the point $B_{0}$ is equal to

$$
\left\{\left(0_{X^{*}}, s^{*}, l^{*} ; 0\right)\right\} \subset\left(X^{*} \times S^{*} \times L^{*}\right) \times T_{\left[x_{0}^{*}: s_{0}^{*}: l_{0}^{*}\right]}^{*}\left(\mathbb{P}_{+}\left(X^{*} \times S^{*} \times L^{*}\right)\right)
$$

where the last zero in the first set belongs to $T_{\left[x_{0}^{*}: s_{0}^{*}: l_{0}^{*}\right]}\left(\mathbb{P}_{+}\left(X^{*} \times S^{*} \times L^{*}\right)\right)$. As in Step 2, the fiber of $\tilde{\Gamma}=\left(d f^{*}\right)_{*} p^{*} \Gamma$ over $B_{0}$ is equal to

$$
\left\{\left(x^{*}, s^{*}, 0_{L^{*}} ; \xi^{*}\right) \mid\left(x^{*}, s^{*} ; \nu\left(\xi^{*}\right)\right) \in\{0\} \cup \Gamma\right\} \text {. }
$$

Hence the intersection of the sets (3.5.17) and (3.5.18) is equal to

$$
\left\{\left(0_{X^{*}}, s^{*}, 0_{L^{*}} ; 0\right) \mid\left(0_{X^{*}}, s^{*} ; 0\right) \in\{0\} \cup \Gamma\right\}=\left\{\left(0_{X^{*}}, s^{*}, 0_{L^{*}} ; 0\right) \mid\left(0_{X^{*}}, s^{*} ; 0\right) \in \Gamma\right\} .
$$


But the last set is empty since the set $\left\{\left(0_{X^{*}}, s^{*} ; 0\right)\right\} \cap \Gamma$ is empty. Indeed by the assumption $f \circ g$ is transversal to $\xi$, and hence the second condition of transversality $\left(T R_{2}\right)$ is satisfied: the intersection of $\Gamma$ with the conormal bundle of $X \times_{X \times S} \mathbb{P}_{X \times S} \subset \mathbb{P}_{X \times S}$ is empty. Step 3 is proved.

Step 4. It remains to show that $(f \circ g)^{*}(\xi)=g^{*}\left(f^{*}(\xi)\right)$.

$\overline{\text { First let }}$ us prove it in the special case $X=Z$, i.e. $S=\{0\}$. In this case we have

$$
Z \stackrel{g}{\rightarrow} X \times L \stackrel{f}{\rightarrow} Z
$$

and $f \circ g=I d_{Z}$. For any closed conic subsets $\Lambda \subset T^{*} Z \backslash \underline{0}, \Gamma \subset T^{*} \mathbb{P}_{Z} \backslash \underline{0}$, we denote, as in Step 2 , by $\tilde{\Gamma}:=\left(d f^{*}\right)_{*} p^{*} \Gamma$. Then the maps

$$
f^{*}: V_{\Lambda, \Gamma}^{-\infty}(Z) \rightarrow V_{f^{*} \Lambda, \tilde{\Gamma}}^{-\infty}(X \times L), g^{*}: V_{f^{*} \Lambda, \tilde{\Gamma}}^{-\infty}(X \times L) \rightarrow V^{-\infty}(Z)
$$

are sequentially continuous operators. In particular taking $\Lambda=T^{*} Z \backslash \underline{0}, \Gamma=T^{*} \mathbb{P}_{Z} \backslash \underline{0}$ we deduce that

$$
g^{*} \circ f^{*}: V^{-\infty}(Z) \rightarrow V^{-\infty}(Z)
$$

is a sequentially continuous operator.

To show that $g^{*} \circ f^{*}=(f \circ g)^{*}(=I d)$ it is enough to check that these operators coincide on characteristic functions of compact submanifolds with corners; indeed if two sequentially continuous linear operators, defined on $V^{-\infty}(Z)$ as a source space, coincide on constructible functions then they coincide by Corollary 1.3 in [10]. Thus let $P \subset Z$ be a compact submanifold with corners. Then by Proposition 3.3.4

$$
f^{*}\left(\Xi_{\mathcal{P}}\left(\mathbb{1}_{P}\right)\right)=\Xi_{\mathcal{P}}\left(\mathbb{1}_{P \times L}\right) .
$$

But observe that $Z$ is transversal to $P \times L$. Hence by Proposition 3.5 .12 we have $g^{*}\left(\Xi_{\mathcal{P}}\left(\mathbb{1}_{P \times L}\right)\right)=$ $\Xi_{\mathcal{P}}\left(\mathbb{1}_{(P \times L) \cap Z}\right)=\Xi_{\mathcal{P}}\left(\mathbb{1}_{Z}\right)$. Hence $g^{*} \circ f^{*}=I d$.

Now let us consider the general case. Let $\xi$ correspond to a pair of currents $(C, T)$. As in Steps 1-3 let us denote $\Lambda:=W F(C), \Gamma:=W F(T)$. From the computations in Steps 1-3 and from the behavior of wave fronts under pull-back and push-forward (see Propositions 1.4.4 and 1.4.10) it follows that $(f \circ g)^{*}$ and $g^{*} \circ f^{*}$ are sequentially continuous linear operators

$$
V_{\Lambda, \Gamma}^{-\infty}(Z) \rightarrow V^{-\infty}(X)
$$

By Lemma 1.6.8 it is enough to show that these operators coincide on the subspace of smooth valuations. Thus we may assume that $\xi$ is a smooth valuation.

Let us decompose $g$ as $X \stackrel{h}{\rightarrow} Z \stackrel{k}{\rightarrow} Y$ where $h(x)=\left(x, 0_{S}\right), k(z)=\left(z, 0_{L}\right)$. Both maps $h, k$ are imbeddings.

3.5.14 Claim. These maps $h, k$ and the valuation $f^{*} \xi \in V^{-\infty}(Y)$ satisfy the assumptions of Proposition 3.5.11.

Let us postpone the proof of this claim and finish the proof of our proposition. By Proposition 3.5.11, $g^{*}\left(f^{*} \xi\right)=h^{*}\left(k^{*}\left(f^{*} \xi\right)\right)$. By what we have just shown $k^{*}\left(f^{*}(\xi)\right)=\xi$. 
Hence $g^{*}\left(f^{*}(\xi)\right)=h^{*} \xi$. On the other hand $f \circ g=h$, hence $(f \circ g)^{*} \xi=h^{*} \xi$. Thus to finish the proof of the proposition it remains to prove Claim 3.5.14.

Proof of Claim 3.5.14. Let $f^{*} \xi \in V^{-\infty}(Y)$ be given by a pair of currents $(\tilde{C}, \tilde{T})$. Since $\xi$ is smooth, $\tilde{C}=f^{*} C$ is also smooth. Thus $W F(\tilde{C})=\emptyset$. The wave front of $\tilde{T}$ is contained in the conormal bundle of $Y \times_{Z} \mathbb{P}_{Z} \subset \mathbb{P}_{Y}$, thus let us denote

$$
M:=T_{Y \times{ }_{Z} \mathbb{P}_{Z}}^{*}\left(\mathbb{P}_{Y}\right) \backslash \underline{0} \subset T^{*} \mathbb{P}_{Y} \backslash \underline{0} .
$$

We have

$$
Y \times_{Z} \mathbb{P}_{Z}=\left\{\left(z, l ;\left[z^{*}: 0_{L^{*}}\right]\right)\right\} \subset \mathbb{P}_{Y}=\mathbb{P}_{Z \times L}
$$

Step 1. First we will check that $k: Z \rightarrow Y$ is transversal to $(\emptyset, M)$, i.e. we have to verify the three conditions of transversality $\left(T R_{1}\right)-\left(T R_{3}\right)$ (see Definition 3.5.1).

The first condition $\left(T R_{1}\right)$ is satisfied for trivial reasons. Let us check $\left(T R_{2}\right)$, namely

$$
M \cap T_{Z \times_{Y} \mathbb{P}_{Y}}^{*}\left(\mathbb{P}_{Y}\right)=\emptyset
$$

We have

$$
Z \times_{Y} \mathbb{P}_{Y}=Z \times\left\{0_{L}\right\} \times \mathbb{P}_{+}\left(Z^{*} \times L^{*}\right)
$$

Fix points $z_{0} \in Z,\left[z_{0}^{*}\right] \in \mathbb{P}_{Z}$. Denote $A_{0}:=\left(z_{0}, 0_{L} ;\left[z_{0}^{*}: 0_{L^{*}}\right]\right) \in Y \times_{Z} \mathbb{P}_{Z}$. The fiber of $M \cup \underline{0}$ over $A_{0}$ is equal to

$$
\left.M\right|_{A_{0}} \cup\{0\}=\left\{0_{Z^{*}}\right\} \times\left\{0_{L^{*}}\right\} \times\left. T_{\mathbb{P}_{+}\left(Z^{*} \times 0_{L^{*}}\right.}^{*}\left(\mathbb{P}_{+}\left(Y^{*}\right)\right)\right|_{\left[z_{0}^{*}: 0_{L^{*}}\right.} .
$$

The fiber of $T_{Z \times_{Y} \mathbb{P}_{Y}}^{*}\left(\mathbb{P}_{Y}\right)$ over $A_{0}$ is equal to

$$
\left.T_{Z \times_{Y} \mathbb{P}_{Y}}^{*}\left(\mathbb{P}_{Y}\right)\right|_{A_{0}}=\left\{0_{Z^{*}}\right\} \times L^{*} \times\{0\}
$$

where the last zero on the right hand side belongs to $T_{\left[z_{0}^{*}: 0_{L^{*}}\right]}^{*}\left(\mathbb{P}_{+}\left(Y^{*}\right)\right)$. Next from (3.5.21) and (3.5.22) we see that

$$
M \cap T_{Z \times_{Y} \mathbb{P}_{Y}}^{*}\left(\mathbb{P}_{Y}\right)=\emptyset
$$

Thus the second condition of transversality $\left(T R_{2}\right)$ is checked.

Let us check the third condition $\left(T R_{3}\right)$, namely

$$
M \cap T_{\mathbb{P}_{+}\left(T_{Z}^{*} Y\right)}^{*}\left(\mathbb{P}_{Y}\right)=\emptyset
$$

We have

$$
\mathbb{P}_{+}\left(T_{Z}^{*} Y\right)=\left\{\left(z, 0_{L} ;\left[0_{Z^{*}}: l^{*}\right]\right)\right\} \subset \mathbb{P}_{Y}
$$

It is clear that $\mathbb{P}_{+}\left(T_{Z}^{*} Y\right) \cap\left(Y \times_{Z} \mathbb{P}_{Z}\right)=\emptyset$. Hence trivially

$$
T_{\mathbb{P}_{+}\left(T_{Z}^{*} Y\right)}^{*}\left(\mathbb{P}_{Y}\right) \cap M=\emptyset
$$


thus the condition $\left(T R_{3}\right)$ is satisfied.

Step 2. Let us check that $h: X \rightarrow Z$ is transversal to $k^{*}\left(f^{*} \xi\right)$. It suffices to check that $h: \overline{X \rightarrow Z}$ is transversal to $\left(\emptyset, \beta_{1 *} \alpha_{1}^{*} M\right)$ where

$$
\mathbb{P}_{Z} \stackrel{\beta_{1}}{\leftarrow} \widetilde{\widetilde{\times}_{Y} \mathbb{P}_{Y}} \stackrel{\alpha_{1}}{\rightarrow} \mathbb{P}_{Y}
$$

is the diagram analogous to (3.5.1) but with $Z \stackrel{k}{\rightarrow} Y$ instead of $X \rightarrow Y$. We will prove a stronger statement: $\beta_{1 *} \alpha_{1}^{*} M=\emptyset$.

Similarly to (3.5.2), let us decompose $\alpha_{1}$ as

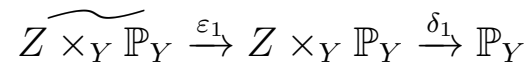

where $\varepsilon_{1}$ is the oriented blow up map along $\mathbb{P}_{+}\left(T_{Z}^{*} Y\right)$, and $\delta_{1}$ is the imbedding map.

Let us consider subset $J \subset \mathbb{P}_{Y}$ defined by

$$
J:=\left(Y \times_{Z} \mathbb{P}_{Z}\right) \cap\left(Z \times_{Y} \mathbb{P}_{Y}\right)=\left\{\left(z, 0_{L} ;\left[z^{*}: 0_{L^{*}}\right]\right)\right\}
$$

where the intersection is obviously transversal. It is clear that

$$
\delta_{1}^{*} M=T_{J}^{*}\left(Z \times_{Y} \mathbb{P}_{Y}\right) \backslash \underline{0} .
$$

Next the oriented blow up map

$$
\varepsilon_{1}: \widetilde{\widetilde{\times}_{Y} \mathbb{P}_{Y}} \rightarrow Z \times_{Y} \mathbb{P}_{Y}
$$

is along the submanifold $\mathbb{P}_{+}\left(T_{Z}^{*} Y\right)$ which obviously does not intersect $J$. It follows that

$$
\varepsilon_{1}^{*} \delta_{1}^{*} M=T_{\varepsilon_{1}^{-1}(J)}^{*}\left(\widetilde{Z \times_{Y} \mathbb{P}_{Y}}\right) \backslash \underline{0},
$$

and $\varepsilon_{1}$ is a diffeomorphism of a neighborhood $\mathcal{O}$ of $\varepsilon_{1}^{-1}(J)$ onto its image. Hence while computing $\beta_{1 *} \varepsilon_{1}^{*} \delta_{1}^{*} M$ we may consider the restriction of $\beta_{1}$ to this neighborhood $\mathcal{O}$. This neighborhood $\mathcal{O}$ can be taken to be

$$
\mathcal{O}:=\left\{\left(z, 0_{L} ;\left[z^{*}: l^{*}\right]\right) \mid z^{*} \neq 0\right\} .
$$

Next $\beta_{1}: \mathcal{O} \rightarrow \mathbb{P}_{Z}$ is given by

$$
\beta_{1}\left(\left(z, 0_{L} ;\left[z^{*}: l^{*}\right]\right)\right)=\left(z ;\left[z^{*}\right]\right) .
$$

Also

$$
\varepsilon_{1}^{-1}(J)=\left\{\left(z, 0_{L} ;\left[z^{*}: 0_{L^{*}}\right]\right)\right\} .
$$

Then clearly the restriction of $\beta_{1}$ to $\varepsilon_{1}^{-1}(J)$ is a diffeomorphism $\varepsilon^{-1}(J) \sim{ }_{\rightarrow} \mathbb{P}_{Z}$. This and the fact that $\beta_{1}: \mathcal{O} \rightarrow \mathbb{P}_{Z}$ is a fibration imply that

$$
\beta_{1 *}\left(T_{\varepsilon_{1}^{-1}(J)}^{*} \mathcal{O} \backslash \underline{0}\right)=\emptyset .
$$

Hence $\beta_{1 *} \alpha_{1 *} M=\beta_{1 *} \varepsilon_{1}^{*} \delta_{1}^{*} M=\emptyset$. Thus Step 2 is completed. 
Step 3. It remains to show that $g=k \circ h$ is transversal to $(\emptyset, M)$. The condition $\left(T R_{1}\right)$ is satisfied trivially.

Let us check $\left(T R_{2}\right)$. Let us suppose in the contrary that there exists a point $A_{0} \in X \times_{Y} \mathbb{P}_{Y}$ and $\left.\xi \in M\right|_{A_{0}}$ which belongs also to the fiber over $A_{0}$ of the conormal bundle $T_{X \times_{Y} \mathbb{P}_{Y}}^{*}\left(\mathbb{P}_{Y}\right)$. The point $A_{0}$ has the form

$$
A_{0}=\left(x_{0}, 0_{S}, 0_{L} ;\left[x_{0}^{*}: s_{0}^{*}: 0_{L^{*}}\right]\right)
$$

Denote also

$$
Q_{0}:=\left[x_{0}^{*}: s_{0}^{*}: 0_{L^{*}}\right] \in \mathbb{P}_{+}\left(X^{*} \times S^{*} \times 0_{L^{*}}\right) \subset \mathbb{P}_{+}\left(Y^{*}\right) .
$$

Using (3.5.21) one sees that $\xi$ must have the form

$$
\xi=\left(0_{X^{*}}, 0_{S^{*}}, 0_{L^{*}} ; \zeta\right) \text { with }\left.\zeta \in\left(T_{\mathbb{P}_{+}\left(X^{*} \times S^{*} \times 0_{L^{*}}\right)}\left(\mathbb{P}_{+}\left(Y^{*}\right)\right)\right)\right|_{Q_{0}} .
$$

But the assumption that $\left.\xi \in\left(T_{X \times_{Y} \mathbb{P}_{Y}}\left(\mathbb{P}_{Y}\right)\right)\right|_{A_{0}}$ implies that $\zeta=0$. Hence $\xi=0$ which is a contradiction. Thus $\left(T R_{2}\right)$ is proved.

Let us prove $\left(T R_{3}\right)$. Let us suppose in the contrary that there exists a point $B_{0} \in$ $\mathbb{P}_{+}\left(T_{X}^{*} Y\right)$ and $\left.\eta \in M\right|_{B_{0}}$ such that $\eta$ also belongs to the fiber over $B_{0}$ of the conormal bundle $T_{\mathbb{P}_{+}\left(T_{X}^{*} Y\right)}^{*}\left(\mathbb{P}_{Y}\right)$. Notice also that

$$
\mathbb{P}_{+}\left(T_{X}^{*} Y\right)=\left\{\left(x, 0_{S}, 0_{L} ;\left[0_{X^{*}}: s^{*}: l^{*}\right]\right)\right\} .
$$

Necessarily $B_{0}$ has the form

$$
B_{0}=\left(\tilde{x}_{0}, 0_{S}, 0_{L} ;\left[0_{X^{*}}: \tilde{s}_{0}^{*}: 0_{L^{*}}\right]\right) .
$$

Let us denote $R_{0}:=\left[0_{X^{*}}: \tilde{s}_{0}^{*}: 0_{L^{*}}\right] \in \mathbb{P}_{+}\left(Y^{*}\right)$. In this notation $\eta$ must have the form

$$
\eta=\left(0_{X^{*}}, 0_{S^{*}}, 0_{L^{*}} ; \tau\right)
$$

with $\left.\left.\tau \in\left(T_{\mathbb{P}_{+}\left(X^{*} \times S^{*} \times 0_{L^{*}}\right)}\left(\mathbb{P}_{+}\left(Y^{*}\right)\right)\right)\right|_{R_{0}} \cap\left(T_{\mathbb{P}_{+}\left(0_{\left.X^{*} \times S^{*} \times L^{*}\right)}^{*}\right.}\left(\mathbb{P}_{+}\left(Y^{*}\right)\right)\right)\right|_{R_{0}}$. But the last intersection is equal to $\{0\}$. This is a contradiction, thus $\left(T R_{3}\right)$ is proved.

Hence Claim 3.5.14 is proved. Hence Proposition 3.5.13 is proved completely too. Q.E.D.

\subsection{Push-forward of generalized valuations under submersions.}

Let $f: X \rightarrow Y$ be a proper submersion. In Section 3.2 we have defined a push-forward map on smooth valuations

$$
f_{*}: V^{\infty}(X) \rightarrow V^{\infty}(Y)
$$

which is a continuous operator. In this section we extend this map to a partially defined map on generalized valuations. This extension will be important for applications to integral geometry in Section 4 below.

Ideologically, the push-forward map $f_{*}$ is dual to the pull-back map $f^{*}$. Proposition 3.3 .3 implies that for the submersion $f: X \rightarrow Y$ we have the continuous pull-back map

$$
f^{*}: V^{\infty}(Y) \rightarrow V_{\emptyset, M}^{-\infty}(X)
$$


where $M \subset T^{*} \mathbb{P}_{X} \backslash \underline{0}$ is the conormal bundle of $X \times_{Y} \mathbb{P}_{Y} \subset \mathbb{P}_{X}$ with the zero section removed. (In this case $f^{*}$ is topologically continuous and not just sequentially continuous. This is due to the fact that the source space $V^{\infty}(Y)$ is metrizable.) If in addition $f$ is proper then $f^{*}$ maps compactly supported valuations to compactly supported:

$$
f^{*}: V_{c}^{\infty}(Y) \rightarrow V_{\emptyset, M, c}^{-\infty}(X) .
$$

Next in [11] there was defined a partial product on generalized valuations on a manifold $X$ which will be needed now. Let $\xi_{1}, \xi_{2} \in V^{-\infty}(X)$ be generalized valuations. Assume that the wave fronts $\left(\Lambda_{i}, \Gamma_{i}\right), i=1,2$, of the currents corresponding to $\xi_{i}$ satisfy some explicitly written condition of disjointness (see Theorem 1.6.7, or (3.6.1)-(3.6.5) below in this section). Then one can define the product $\xi_{1} \cdot \xi_{2} \in V^{-\infty}(X)$. Moreover this product defines a bilinear jointly sequentially continuous map

$$
V_{\Lambda_{1}, \Gamma_{1}}^{-\infty}(X) \times V_{\Lambda_{2}, \Gamma_{2}}^{-\infty}(X) \rightarrow V^{-\infty}(X)
$$

which coincides with the usual product on smooth valuations.

Now in order to define the push-forward map $f_{*}$ for a proper submersion $f: X \rightarrow Y$, let us fix an arbitrary closed conic subsets

$$
\Lambda \subset T^{*} X \backslash \underline{0}, \Gamma \subset T^{*} \mathbb{P}_{X} \backslash \underline{0}
$$

such that $(\Lambda, \Gamma)$ and $(\emptyset, M)$ satisfy the above mentioned condition of disjointness. Let us define eventually

$$
f_{*}: V_{\Lambda, \Gamma}^{-\infty}(X) \rightarrow V^{-\infty}(Y)
$$

as follows

$$
<f_{*} \xi, \phi>:=\int_{X} \xi \cdot f^{*} \phi
$$

for any $\xi \in V_{\Lambda, \Gamma}^{-\infty}(X)$ and any test valuation $\phi \in V_{c}^{\infty}(Y)$. The above discussion implies that we get a sequentially continuous linear operator $f_{*}$.

By the discussion in Section 3.3 it is clear that the restriction of this $f_{*}$ to smooth valuations coincides with the push-forward defined in Section 3.2. Thus there is no abuse of notation.

Let us remind again the condition of disjointness of wave fronts. Assume that $\Lambda_{i} \subset$ $T^{*} Y \backslash \underline{0}, \Gamma_{i} \subset T^{*} \mathbb{P}_{Y} \backslash \underline{0}, i=1,2$, be two closed conic subsets. One can define a jointly sequentially continuous bilinear map

$$
V_{\Lambda_{1}, \Gamma_{1}}^{-\infty}(Y) \times V_{\Lambda_{2}, \Gamma_{2}}^{-\infty}(Y) \rightarrow V^{-\infty}(Y)
$$

extending the product on smooth valuations, provided the following conditions are satisfied:

$$
\begin{array}{r}
\Lambda_{1} \cap s\left(\Lambda_{2}\right)=\emptyset, \\
\Gamma_{1} \cap s\left(\pi_{Y}^{*} \Lambda_{2}\right)=\emptyset, \\
\Gamma_{2} \cap s\left(\pi_{Y}^{*} \Lambda_{1}\right)=\emptyset, \\
\quad \text { if }\left(y,\left[\xi_{i}\right], u_{i}, 0\right) \in \Gamma_{i}, i=1,2, \text { then } u_{1} \neq-u_{2}, \\
\text { if }(y,[\xi]) \in \mathbb{P}_{Y} \text { and }\left.\left(u, \eta_{1}\right) \in \Gamma_{1}\right|_{(y,[\xi])},\left.\left(-u, \eta_{2}\right) \in \Gamma_{2}\right|_{(y,[-\xi]), \text { then }} \\
d \theta^{*}\left(0, \eta_{1}, \eta_{2}\right) \neq(0, l,-l) \in T_{(y,[\xi],[\xi])}^{*}\left(\mathbb{P}_{Y} \times_{Y} \mathbb{P}_{Y}\right)
\end{array}
$$

where $\theta: \mathbb{P}_{Y} \times_{Y} \mathbb{P}_{Y} \rightarrow \mathbb{P}_{Y} \times_{Y} \mathbb{P}_{Y}$ is defined by $\theta\left(y,\left[\zeta_{1}\right],\left[\zeta_{2}\right]\right)=\left(y,\left[\zeta_{1}\right],\left[-\zeta_{2}\right]\right)$. 
Now let us define the push-forward map

$$
f_{*}: V_{\Lambda, \Gamma}^{-\infty}(X) \rightarrow V^{-\infty}(Y)
$$

for a proper submersion $f: X \rightarrow Y$ and closed conic subsets $\Lambda \subset T^{*} X \backslash \underline{0}, \Gamma \subset T^{*} \mathbb{P}_{X} \backslash \underline{0}$ such that

$$
\text { the pairs }(\Lambda, \Gamma) \text { and }\left(\emptyset, T_{X \times_{Y} \mathbb{P}_{Y}}\left(\mathbb{P}_{X}\right)\right) \text { satisfy (3.6.1)-(3.6.5), }
$$

as follows:

$$
<f_{*} \phi, \psi>=\int_{Y} \phi \cdot f^{*} \psi
$$

for any $\phi \in V_{\Lambda, \Gamma}^{-\infty}(X), \psi \in V_{c}^{\infty}(Y)$. The previous discussion implies readily that this map $f_{*}: V_{\Lambda, \Gamma}^{-\infty}(X) \rightarrow V^{-\infty}(Y)$ is a sequentially continuous linear operator.

Under the same assumption on $(\Lambda, \Gamma)$ one defines in the same way the push-forward on compactly supported valuations

$$
f_{*}: V_{\Lambda, \Gamma, c}^{-\infty}(X) \rightarrow V_{c}^{-\infty}(Y)
$$

if $f: X \rightarrow Y$ is a submersion, but not necessarily proper.

In order to study further properties of the push-forward map, let us prove some technical results.

Assume now that $i: X \hookrightarrow Y$ be a smooth imbedding. Recall that we have the diagram $(\operatorname{see}(3.1 .3))$

$$
\mathbb{P}_{X} \stackrel{\beta}{\leftarrow} \widetilde{\times_{Y} \mathbb{P}_{Y}} \stackrel{\alpha}{\rightarrow} \mathbb{P}_{Y} .
$$

Recall that by Proposition 3.4 .2 for $\phi \in V_{c}^{-\infty}(X)$ given by a pair of currents $\left(C_{\phi}, T_{\phi}\right)$ the push-forward $i_{*} \phi$ is given by the pair $\left(0, \alpha_{*} \beta^{*} T_{\phi}\right)$.

Recall also that, by the Definition of the pull-back under immersions from Section 3.5 , if $\psi \in V^{-\infty}(Y)$ is given by a pair $\left(C_{\psi}, T_{\psi}\right)$ and the imbedding $i$ is transversal to $\psi$ then the pull-back $i^{*} \psi$ is given by the pair of currents $\left(i^{*} C_{\psi}, \beta_{*} \alpha^{*} T_{\psi}\right)$.

Let us denote also

$$
\begin{aligned}
\Lambda_{\phi} & :=W F\left(C_{\phi}\right), \Lambda_{\psi}:=W F\left(C_{\psi}\right), \\
\Gamma_{\phi} & :=W F\left(T_{\phi}\right), \Gamma_{\psi}:=W F\left(T_{\psi}\right) .
\end{aligned}
$$

3.6.1 Lemma. Using the above notation let us assume that the imbedding $i: X \hookrightarrow Y$ is transversal to $\psi \in V^{-\infty}(Y)$ (in the sense of Definition 3.5.2), the pairs of subsets $\left(\Lambda_{\phi}, \Gamma_{\phi}\right)$ and $\left(i^{*} \Lambda_{\psi}, \beta_{*} \alpha^{*} \Gamma_{\psi}\right)$ satisfy (3.6.1)-(3.6.5), and also the pairs of sets $\left(\emptyset, \alpha_{*} \beta^{*} \Gamma_{\phi}\right)$ and $\left(\Lambda_{\psi}, \Gamma_{\psi}\right)$ satisfy (3.6.1)-(3.6.5).

Then $i_{*} \phi \cdot \psi$ and $\phi \cdot i^{*} \psi$ are well defined, and

$$
\int_{Y} i_{*} \phi \cdot \psi=\int_{X} \phi \cdot i^{*} \psi .
$$


Proof. The well-definedness of the expressions is clear. This implies that the two bilinear maps $V_{\Lambda_{\phi}, \Gamma_{\phi}, c}^{-\infty}(X) \times V_{\Lambda_{\psi}, \Gamma_{\psi}}^{-\infty}(Y) \rightarrow \mathbb{C}$ given respectively by $(\mu, \nu) \mapsto \int_{Y} i_{*} \mu \cdot \nu$ and $(\mu, \nu) \mapsto \int_{X} \mu \cdot i^{*} \nu$ are jointly sequentially continuous. Thus in order to show that they coincide it is enough to assume that $\psi$ is smooth. But for smooth $\psi$ the equality (3.6.7) is satisfied by the definition of $i_{*}$ from Section 3.4. Q.E.D.

3.6.2 Lemma. Let $Y=X \times Z, f: X \times Z \rightarrow X$ be the natural projection. Let $i: X \hookrightarrow$ $X \times Z$ be a closed imbedding of the form $i(x)=\left(x, z_{0}\right)$ where $z_{0} \in Z$ is a fixed point. Let $\phi \in V_{c}^{-\infty}(X)$ be a generalized valuation with compact support. Let $\xi \in V^{\infty}(X)$ be a smooth valuation.

Then $\phi, \psi:=f^{*} \xi$, and $i: X \hookrightarrow Y$ satisfy the assumptions of Lemma 3.6.1. Consequently the generalized valuations $i_{*} \phi \cdot f^{*} \xi$ and $\phi \cdot i^{*}\left(f^{*} \xi\right)$ are well defined, and

$$
\int_{Y} i_{*} \phi \cdot f^{*} \xi=\int_{X} \phi \cdot i^{*}\left(f^{*} \xi\right) .
$$

Proof. Let $\psi:=f^{*} \xi$ be given by the pair of currents $\left(C_{\psi}, T_{\psi}\right)$. Since $\xi$ is smooth we have

$$
\begin{array}{r}
\Lambda_{\psi}=\emptyset, \\
\Gamma_{\psi} \subset T_{Y{ }_{X} \mathbb{P}_{X}}^{*}\left(\mathbb{P}_{Y}\right) .
\end{array}
$$

The condition (3.6.9) implies readily that $\beta_{*} \alpha^{*} \Gamma_{\psi}=\emptyset$. Hence the first assumption of Lemma 3.6.1 follows: the pairs $\left(\Lambda_{\phi}, \Gamma_{\phi}\right)$ and $\left(i^{*} \Lambda_{\psi}, \beta_{*} \alpha^{*} \Gamma_{\psi}\right)=(\emptyset, \emptyset)$ satisfy (3.6.1)-(3.6.5).

Let us check now the second assumption, namely that the pairs $\left(\emptyset, \alpha_{*} \beta^{*} \Gamma_{\phi}\right)$ and $\left(\Lambda_{\psi}, \Gamma_{\psi}\right)=$ $\left(\emptyset, \Gamma_{\psi}\right)$ satisfy (3.6.1)-(3.6.5). The conditions (3.6.1)-(3.6.3) are satisfied trivially.

Let us check (3.6.4). Assume that $\left(y,\left[\xi_{1}\right], u_{1}, 0\right) \in \alpha_{*} \beta^{*} \Gamma_{\phi},\left(y,\left[\xi_{2}\right], u_{2}, 0\right) \in \Gamma_{\psi}$. But (3.6.9) implies that $u_{2}=0$ which is a contradiction. Thus (3.6.4) is proved.

Let us check (3.6.5). For simplicity of the notation we may and will assume that $X, Z$ are vector spaces, $z_{0}=0_{Z} \in Z$. Assume that

$$
\begin{array}{r}
(y,[\xi]) \in \mathbb{P}_{Y}, \\
\left.\left(u, \eta_{1}\right) \in\left(\alpha_{*} \beta^{*} \Gamma_{\phi}\right)\right|_{(y,[\xi])}, \\
\left.\left(-u, \eta_{2}\right) \in \Gamma_{\psi}\right|_{(y,[-\xi])} .
\end{array}
$$

Clearly $y=\left(x, 0_{Z}\right)$. Next (3.6.9) implies that $u=0$ and $\xi=\left(x^{*}, 0_{Z^{*}}\right)$ with $x^{*} \in X^{*}$. Denote $\left(0, \eta_{2}^{\prime}\right):=\left.d s^{*}\left(\left(0, \eta_{2}\right)\right) \in\left(T_{Y \times X}^{*} \mathbb{P}_{X}\left(\mathbb{P}_{Y}\right)\right)\right|_{(x,[\xi]}$. Thus we have to show that $\eta_{1} \neq-\eta_{2}^{\prime}$.

Otherwise we would have that

$$
\left.\left(0, \eta_{1}\right) \in\left(\alpha_{*} \beta^{*} \Gamma_{\phi} \cap T_{Y \times_{X} \mathbb{P}_{X}}^{*}\left(\mathbb{P}_{Y}\right)\right)\right|_{(y,[\xi])} .
$$

Since $[\xi]=\left[x^{*}: 0_{Z^{*}}\right]$, there exists a neighborhood $U \subset \mathbb{P}_{Y}$ of $(y,[\xi])$ such that $\alpha: \alpha^{-1}(U)$ $\rightarrow U$ is a diffeomorphism. Thus we will simply identify $\alpha^{-1}(U)$ with $U$ via $\alpha$ (recall that $\alpha$ is the oriented blow up map along $\left.\mathbb{P}_{+}\left(T_{X}^{*} Y\right) \subset \mathbb{P}_{Y}\right)$. Then there exists a cotangent vector 
$\left.\lambda \in\left(T^{*} \mathbb{P}_{X}\right)\right|_{\left(x,\left[x^{*}\right]\right)}$ such that $\left(0, \eta_{1}\right)=d \beta^{*}(\lambda)$. Let us denote by $F$ the tangent space at $(y,[\xi])$ to the fiber $\beta^{-1}\left(\left(x,\left[x^{*}\right]\right)\right)$. Then $\left(0, \eta_{1}\right)$ vanishes on $F$. Also by $(3.6 .10)\left(0, \eta_{1}\right)$ vanishes on the tangent space of $Y \times_{X} \mathbb{P}_{X}$. The latter space is clearly transversal to $F$. Hence $\left(0, \eta_{1}\right)$ vanishes, which is a contradiction. Q.E.D.

3.6.3 Corollary. As in Lemma 3.6.2, let $Y=X \times Z, f: X \times Z \rightarrow X$ be the natural projection. Let $i: X \hookrightarrow X \times Z$ be a closed imbedding of the form $\left[x \mapsto\left(x, z_{0}\right)\right]$ where $z_{0} \in Z$ is a fixed point. Let $\phi \in V_{c}^{-\infty}(X)$ be a generalized valuation with compact support. Let $\xi \in V^{\infty}(X)$ be a smooth valuation. Then

$$
\int_{Y} i_{*} \phi \cdot f^{*} \xi=\int_{X} \phi \cdot \xi
$$

where all the products are automatically well defined.

Proof. By Lemma 3.6 .2 the products are well defined, and

$$
\int_{Y} i_{*} \phi \cdot f^{*} \xi=\int_{X} \phi \cdot i^{*}\left(f^{*} \xi\right)
$$

But Proposition 3.5.13 implies immediately that $i^{*}\left(f^{*} \xi\right)=\xi$. Corollary is proved. Q.E.D.

Our next goal is to show that the pushforward under a submersion of a constructible function (considered as a generalized valuation) is equal to integration of this function with respect to the Euler characteristic along the fibers (see Ch. 9 of [27] for the detailed discussion of this notion). However we will do this explicitly only under rather restrictive assumptions. This generality will be sufficient for some integral geometric applications discussed below, but from the point of view of the general theory it does not seem to be very satisfactory. Our first result in this direction the following lemma.

3.6.4 Lemma. Let $X, Z$ be smooth manifolds without boundary, let $Y=X \times Z$. Let $f: X \times Z \rightarrow X$ be the natural projection. Let $P \in \mathcal{P}(Z)$. Then the wave fronts of the generalized valuation $\Xi_{\mathcal{P}}\left(\mathbb{1}_{X \times P}\right) \in V^{-\infty}(Y)$ satisfy the condition of transversality (3.6.6) (note that here the roles of $X$ and $Y$ are interchanged) and

$$
f_{*}\left(\Xi_{\mathcal{P}}\left(\mathbb{1}_{X \times P}\right)\right)=\chi(P) \cdot \chi \text {. }
$$

Proof. We have $N(X \times P)=Y \times_{Z} N(P) \subset Y \times_{Z} \mathbb{P}_{Z} \subset \mathbb{P}_{Y}$. Clearly $N(X \times P)$ is disjoint from $Y \times_{X} \mathbb{P}_{X}$. Hence the push-forward $f_{*}\left(\Xi_{\mathcal{P}}\left(\mathbb{1}_{X \times P}\right)\right)$ is well defined, i.e. the condition of transversality (3.6.6) is satisfied.

Triangulating $P$ we may assume that $Z=\mathbb{R}^{l}$ and $P$ is a linear simplex. For $N \in \mathbb{N}$ let us denote $P_{N}:=\frac{1}{N} \cdot P$. Also let us denote for brevity

$$
\begin{array}{r}
\Lambda:=T^{*} Y \backslash \underline{0}, \\
\Gamma:=\left(Y \times_{Z} \mathbb{P}_{Z}\right) \times_{\mathbb{P}_{Y}}\left(T^{*} \mathbb{P}_{Y} \backslash \underline{0}\right) \subset T^{*} \mathbb{P}_{Y} .
\end{array}
$$

It is easy to see that the pairs of sets $(\Lambda, \Gamma)$ and $\left(\emptyset, T_{Y \times_{X} \mathbb{P}_{X}}^{*} \mathbb{P}_{Y} \backslash \underline{0}\right)$ satisfy the conditions (3.6.1)-(3.6.5) . 
Below we will show that

$$
\Xi_{\mathcal{P}}\left(\mathbb{1}_{X \times P_{N}}\right) \rightarrow \Xi_{\mathcal{P}}\left(\mathbb{1}_{X \times\left\{0_{Z}\right\}}\right) \text { in } V_{\Lambda, \Gamma}^{-\infty}(Y) \text { as } N \rightarrow \infty .
$$

First let us finish the proof of lemma. Evidently $f_{*}\left(\Xi_{\mathcal{P}}\left(\mathbb{1}_{X \times P_{N}}\right)\right)=f_{*}\left(\Xi_{\mathcal{P}}\left(\mathbb{1}_{X \times P}\right)\right)$ for any $N$. Hence by (3.6.11) $f_{*}\left(\Xi_{\mathcal{P}}\left(\mathbb{1}_{X \times P}\right)\right)=f_{*}\left(\Xi_{\mathcal{P}}\left(\mathbb{1}_{X \times\left\{0_{Z}\right\}}\right)\right)$. Let $i: X \hookrightarrow X \times Z$ be the imbedding $i(x)=\left(x, 0_{Z}\right)$. Let $\phi:=\Xi_{\mathcal{P}}\left(\mathbb{1}_{X}\right) \in V^{-\infty}(X)$. Applying Corollary 3.6.3 to $\phi, f$, and $i$ we deduce the result (formally we may assume that $X$ is compact).

Thus it remains to prove (3.6.11). It is clear that $\left[\left[X \times P_{N}\right]\right] \rightarrow\left[\left[X \times\left\{0_{Z}\right\}\right]\right]$ in $C_{\Lambda}^{-\infty}(Y)=$ $C^{-\infty}(Y)$. Next $N\left(X \times P_{N}\right)=X \times N\left(P_{N}\right) \subset \mathbb{P}_{Y}$. By [12], Lemma 2.1.3, we have $\left[\left[N\left(P_{N}\right)\right]\right]$ $\rightarrow\left[\left[N\left(\left\{0_{Z}\right\}\right)\right]\right]$ in $\mathcal{D}_{\operatorname{dim} Z-1}\left(\mathbb{P}_{Z}\right)$. Hence $\left[\left[X \times N\left(P_{N}\right)\right]\right] \rightarrow\left[\left[X \times N\left(\left\{0_{Z}\right\}\right)\right]\right]=\left[\left[N\left(X \times\left\{0_{Z}\right\}\right)\right]\right]$ in $\mathcal{D}_{\operatorname{dim}_{Y}-1}\left(\mathbb{P}_{Y}\right)$.

It remains to show a seemingly more precise statement that $\left[\left[N\left(X \times P_{N}\right)\right]\right] \rightarrow[[N(X \times$ $\left.\left.\left.\left\{0_{Z}\right\}\right)\right]\right]$ in $C_{\Gamma}^{-\infty}\left(\mathbb{P}_{Y}, \Omega^{\operatorname{dim} Y}\right)$, which is obvious since for any $N \in \mathbb{N}$ one has an inclusion $N\left(X \times P_{N}\right) \subset Y \times_{Z} \mathbb{P}_{Z}$. Lemma is proved. Q.E.D.

3.6.5 Proposition. Let $X=\mathbb{R}^{m}, Z=\mathbb{R}^{n-m}, Y=X \times Z=\mathbb{R}^{n}$. Let $f: Y \rightarrow X$ be the natural projection. Let $A \subset Y$ be a compact convex set with smooth boundary and strictly positive Gauss curvature. Then the push-forward $f_{*}\left(\Xi_{\mathcal{P}}\left(\mathbb{1}_{A}\right)\right)$ is well defined and is equal to $\Xi_{\mathcal{P}}\left(\mathbb{1}_{f(A)}\right)$.

Proof. Clearly we may and will assume that $n>m$. The Gauss map $\partial A \rightarrow S^{n-1}$ is a diffeomorphism. This implies that $N(A)$ is transversal to $Y \times_{X} \mathbb{P}_{X}$. Hence the wave fronts of $\Xi_{\mathcal{P}}\left(\mathbb{1}_{A}\right)$ satisfy the condition (3.6.6) (with $X$ and $Y$ interchanged). Hence $f_{*}\left(\Xi_{\mathcal{P}}\left(\mathbb{1}_{A}\right)\right)$ is well defined.

For $\varepsilon \in\left\{1, \frac{1}{2}, \frac{1}{3}, \frac{1}{4}, \ldots\right\} \cup\{0\}$ let us denote by $f_{\varepsilon}$ the linear map $\mathbb{R}^{n} \rightarrow \mathbb{R}^{n}$ which is given by $f_{\varepsilon}(y, z)=(y, \varepsilon z)$. For $\varepsilon>0$ let us denote by $g_{\varepsilon}: \mathbb{P}_{\mathbb{R}^{n}} \rightarrow \mathbb{P}_{\mathbb{R}^{n}}$ the induced map. Denote also $A_{\varepsilon}:=f_{\varepsilon}(A)$. Notice that $A_{0}=f(A) \times\left\{0_{Z}\right\}$.

Let us fix a small open conic neighborhood $\mathcal{U} \subset T^{*} \mathbb{P}_{Y} \backslash \underline{0}$ of the set $T_{Y \times_{X} \mathbb{P}_{X}} \mathbb{P}_{Y} \backslash \underline{0}$ (it will be clear from the discussion below how small $\mathcal{U}$ should be). Denote by $\Gamma$ the complement of $\mathcal{U}$ in $T^{*} \mathbb{P}_{Y} \backslash \underline{0}$. Denote $\Lambda:=T^{*} Y \backslash \underline{0}$. First it is easy to see that the pair of sets $(\Lambda, \Gamma)$ satisfies the condition (3.6.6). We will show that

$$
\Xi_{\mathcal{P}}\left(\mathbb{1}_{A_{\varepsilon}}\right) \rightarrow \Xi_{\mathcal{P}}\left(\mathbb{1}_{A_{0}}\right) \text { in } V_{\Lambda, \Gamma}^{-\infty}(Y) \text { as } \varepsilon \rightarrow+0 .
$$

This, sequential continuity of $f_{*}: V_{\Lambda, \Gamma}^{-\infty}(Y) \rightarrow V^{\infty}$, and Corollary 3.6 .3 will imply our lemma easily.

Thus it remains to prove (3.6.12). It is clear that $A_{\varepsilon} \rightarrow A_{0}$ in the Hausdorff metric. Hence by [12], Lemma 2.1.3, [[N( $\left.\left.\left.A_{\varepsilon}\right)\right]\right] \rightarrow\left[\left[N\left(A_{0}\right)\right]\right]$ in $\mathcal{D}_{n-1}\left(\mathbb{P}_{Y}\right)$. It is also clear that $\left[\left[A_{\varepsilon}\right]\right]$ $\rightarrow\left[\left[A_{0}\right]\right]=0$ in $\mathcal{D}_{n}(Y)=C^{-\infty}(Y)$. Hence $\Xi_{\mathcal{P}}\left(\mathbb{1}_{A_{\varepsilon}}\right) \rightarrow \Xi_{\mathcal{P}}\left(\mathbb{1}_{A_{0}}\right)$ in $V^{-\infty}(Y)$.

Thus it remains to show that $\left[\left[N\left(A_{\varepsilon}\right)\right]\right] \rightarrow\left[\left[N\left(A_{0}\right)\right]\right]$ in $C_{\Gamma}^{-\infty}\left(\mathbb{P}_{Y}, \Omega^{n}\right)$ as $\varepsilon \rightarrow+0$. Let us fix a conic subset $K \subset \mathcal{U}$ such that $K / \mathbb{R}_{>0}$ is compact, and fix also a point $s_{0} \in \mathbb{P}_{Y}$. We have to show that there exists a coordinate system $\left\{\left(t_{1}, \ldots, t_{2 n-1}\right)\right\}$ in a neighborhood $\mathcal{T}$ of 
$s_{0}$ such that for any smooth $(n-1)$-form $\omega$ with compact support contained in $\mathcal{T}$, and for any $N \in \mathbb{N}$ one has

$$
\lim _{\varepsilon \rightarrow+0} \sup _{\xi \in K}\left(\left|\int_{N\left(A_{\varepsilon}\right)} e^{i \sum_{\alpha=1}^{2 n-1} t_{\alpha} \xi_{\alpha}} \omega(t)-\int_{N\left(A_{0}\right)} e^{i \sum_{\alpha=1}^{2 n-1} t_{\alpha} \xi_{\alpha}} \omega(t)\right| \cdot\left(1+|\xi|^{N}\right)\right)=0
$$

where the integrations are with respect to $t$.

Since $N\left(A_{\varepsilon}\right)$ converges to $N\left(A_{0}\right)$ in the Hausdorff metric, we may and will assume that $s_{0} \in N\left(A_{0}\right)$. Next since we may restrict our considerations to a neighborhood of $Y \times_{X} \mathbb{P}_{X}$ in $\mathbb{P}_{Y}$, we may and will assume in addition that $s_{0} \in Y \times_{X} \mathbb{P}_{X}$. Thus we assume that $s_{0} \in\left(Y \times_{X} \mathbb{P}_{X}\right) \cap N\left(A_{0}\right)$.

Then we can write $s_{0}=\left(y_{0},\left[n_{0}\right]\right)$ where $y_{0} \in \partial A_{0}, n_{0} \in X^{*} \times\left\{0_{Z^{*}}\right\} \subset Y^{*}$. Let $\tilde{y} \in \partial A$ be the only point on the boundary of $A$ such that the outer normal at $\tilde{y}$ is equal to $n_{0}$.

Let us introduce coordinates $\left\{\left(x_{1}, \ldots, x_{m}\right)\right\}$ on $X$ such that $(0, \ldots, 0)$ corresponds to $0_{X} \in X$, and the axis $x_{m}$ is parallel to $n_{0}$, but has opposite orientation. Let us also choose coordinates $\left\{\left(z_{1}, \ldots, z_{n-m}\right)\right\}$ on $Z$ such that $(0, \ldots, 0)$ corresponds to $0_{Z}$.

Then in a small neighborhood $\mathcal{V}$ of $\tilde{y}$ there exists a smooth strictly convex function $F\left(x_{1}, \ldots, x_{m-1} ; z_{1}, \ldots, z_{n-m}\right)$ such that in this neighborhood $\mathcal{V}$ the set $A$ coincides with the set $\left\{x_{m} \geq F\left(x_{1}, \ldots, x_{m-1} ; z_{1}, \ldots, z_{n-m}\right)\right\}$. Let us denote for brevity

$$
\begin{aligned}
\bar{x}:=\left(x_{1}, \ldots, x_{m-1}\right), z=\left(z_{1}, \ldots, z_{n-m}\right), \\
\nabla_{\bar{x}} F:=\left(\frac{\partial F}{\partial x_{1}}, \ldots, \frac{\partial F}{\partial x_{m-1}}\right), \\
\nabla_{z} F:=\left(\frac{\partial F}{\partial z_{1}}, \ldots, \frac{\partial F}{\partial z_{n-m}}\right) .
\end{aligned}
$$

Then, in $\mathcal{V}$, the normal cycle $N(A)$ is given by

$$
N(A)=\left\{\left(\bar{x}, F(\bar{x}, z), z ;\left[\nabla_{\bar{x}} F(\bar{x}, z):-1: \nabla_{z} F(\bar{x}, z)\right]\right)\right\} .
$$

Then obviously

$$
N\left(A_{\varepsilon}\right)=\left\{\left(\bar{x}, F(\bar{x}, z), \varepsilon z ;\left[\nabla_{\bar{x}} F(\bar{x}, z):-1: \varepsilon^{-1} \nabla_{z} F(\bar{x}, z)\right]\right)\right\} .
$$

Let us consider the change of coordinates $(\bar{x}, z) \mapsto\left(\bar{x}, \nabla_{z} F\right)$. It is locally invertible. Indeed the Jacobian is equal to $\left[\begin{array}{cc}I d_{m-1} & * \\ 0 & \left(\frac{\partial^{2} F}{\partial z_{i} \partial z_{j}}\right)\end{array}\right]$ and the matrix $\left(\frac{\partial^{2} F}{\partial z_{i} \partial z_{j}}\right)$ is invertible since $F$ is strictly convex.

Let us denote $w:=\nabla_{z} F$, and rewrite the parametrization of $N\left(A_{\varepsilon}\right)$ in coordinates $(\bar{x}, w)$. Denote

$$
\begin{array}{r}
G(\bar{x}, w):=F(\bar{x}, z), \\
H(\bar{x}, w):=\nabla_{\bar{x}} F(\bar{x}, z), \\
K(\bar{x}, w):=z
\end{array}
$$

Then

$$
N\left(A_{\varepsilon}\right)=\left\{\left(\bar{x}, G(\bar{x}, w), \varepsilon K(\bar{x}, w) ;\left[H(\bar{x}, w):-1: \varepsilon^{-1} w\right]\right)\right\}
$$


In our fixed small neighborhood $\mathcal{T}$ of $s_{0} \in \mathbb{P}_{Y}$ we can reparameterize $N\left(A_{\varepsilon}\right)$ by choosing $\theta:=\varepsilon^{-1} w$. Then

$$
N\left(A_{\varepsilon}\right)=\{(\bar{x}, G(\bar{x}, \varepsilon \theta), \varepsilon K(\bar{x}, \varepsilon \theta) ;[H(\bar{x}, \varepsilon \theta):-1: \theta])\} .
$$

To abbreviate the notation, now we will write points given in homogeneous coordinates as $[a:-1: b]$, in linear coordinates as $(a, b)$. Then in appropriate coordinate chart on $\mathbb{P}_{Y}$ the normal cycle $N\left(A_{\varepsilon}\right)$ is locally parameterized by $(\bar{x}, \theta)$ as

$$
N\left(A_{\varepsilon}\right)=\{(\bar{x}, G(\bar{x}, \varepsilon \theta), \varepsilon K(\bar{x}, \varepsilon \theta) ; H(\bar{x}, \varepsilon \theta), \theta)\} .
$$

In the same coordinate chart the normal cycle of the limiting set $A_{0}$ is parameterized by $(\bar{x}, \theta)$ as

$$
N\left(A_{0}\right)=\{(\bar{x}, G(\bar{x}, 0), 0 ; H(\bar{x}, 0), \theta)\} .
$$

Applying an appropriate diffeomorphism of the ambient space $\mathbb{P}_{Y}$, we may get the following parametrization of the normal cycles:

$$
\begin{array}{r}
N\left(A_{\varepsilon}\right)=\{(\bar{x}, \bar{G}(\bar{x}, \theta, \varepsilon), \bar{K}(\bar{x}, \theta, \varepsilon) ; \bar{H}(\bar{x}, \theta, \varepsilon), \theta)\}, \\
N\left(A_{0}\right)=\{(\bar{x}, 0,0 ; 0, \theta)\},
\end{array}
$$

where $\bar{G}, \bar{K}, \bar{H}$ are some $C^{\infty}$-smooth functions of $\bar{x}, \theta$, and $\varepsilon \in[0,1]$, such that

$$
\bar{G}(\bar{x}, \theta, \varepsilon=0)=\bar{H}(\bar{x}, \theta, \varepsilon=0)=\bar{K}(\bar{x}, \theta, \varepsilon=0)=0 .
$$

To simplify the notation we will denote

$$
(\bar{G}, \bar{H}, \bar{K})=: M,(\bar{x}, \theta)=: v
$$

Notice that $M$ takes values in $\mathbb{R}^{n}, v \in \mathbb{R}^{n-1}$. Clearly $M$ is $C^{\infty}$-smooth in $(v, \varepsilon)$, and $M(v, 0)=0$.

In this notation we have

$$
\begin{array}{r}
N\left(A_{0}\right)=\left\{\left(v, 0_{\mathbb{R}^{n}}\right)\right\}, \\
N\left(A_{\varepsilon}\right)=\{(v, M(v, \varepsilon))\} .
\end{array}
$$

From this description it is clear that the conic neighborhood $\mathcal{U}$ of $T_{Y{ }_{\times} \mathbb{P}_{X}}^{*} \mathbb{P}_{Y} \backslash \underline{0}$ can be chosen so small that $T_{N\left(A_{\varepsilon}\right)}^{*} \mathbb{P}_{Y} \backslash \underline{0} \subset \Gamma$ for all $\varepsilon \in[0,1]$ (recall that $\Gamma$ is the complement of $\mathcal{U}$ ).

Let us fix an arbitrary conic closed subset $T \subset\left(\mathbb{R}^{n-1} \times \mathbb{R}^{n}\right)^{*} \backslash\{0\}$ such that $T / \mathbb{R}_{>0}$ is compact and

$$
T \cap\left(\left\{0_{\left(\mathbb{R}^{n-1}\right)^{*}}\right\} \times \mathbb{R}^{n *}\right)=\emptyset .
$$

In order to finish the proof of the proposition, i.e. of (3.6.13), we have to show that for any smooth compactly supported $(n-1)$-form $\omega$ on $\mathbb{R}^{2 n-1}$ and any $N \in \mathbb{N}$ one has

$$
\lim _{\varepsilon \rightarrow+0} \sup _{\xi \in T}\left(\left|\int_{N\left(A_{\varepsilon}\right)} e^{i<\xi, y>} \omega(y)-\int_{N\left(A_{0}\right)} e^{i<\xi, y>} \omega(y)\right| \cdot\left(1+|\xi|^{N}\right)\right)=0 .
$$


Let us show that

$$
\varlimsup_{\varepsilon \longrightarrow+0} \sup _{\xi \in T}\left(\left|\int_{N\left(A_{\varepsilon}\right)} e^{i<\xi, y>} \omega(y)\right| \cdot\left(1+|\xi|^{N}\right)\right)<\infty .
$$

We will write $\xi=(\eta, \zeta)$ with $\eta \in\left(\mathbb{R}^{n-1}\right)^{*}, \zeta \in\left(\mathbb{R}^{n}\right)^{*}$. Let us denote also by $\omega_{\varepsilon}$ the pull-back of $\omega$ under the map $v \mapsto(v, M(v, \varepsilon))$. Thus $\omega_{\varepsilon}$ is an $(n-1)$-form on $\mathbb{R}^{n-1}$. In this notation (3.6.18) is rewritten

$$
\varlimsup_{\varepsilon \longrightarrow+0} \sup _{\xi \in T}\left(\left|\int_{\mathbb{R}^{n-1}} e^{i(<\eta, v>+<\zeta, M(v, \varepsilon)>)} \omega_{\varepsilon}(v)\right| \cdot\left(1+(|\eta|+|\zeta|)^{N}\right)\right)<\infty .
$$

The assumption (3.6.16) implies that there exists a constant $C$ such that for any $\xi=$ $(\eta, \zeta) \in T$ one has

$$
|\zeta| \leq C|\eta|
$$

Subdividing $T$ into finitely many parts one may assume that there exists $i_{0} \in\{1, \ldots, n-1\}$ such that for any $(\eta, \zeta) \in T$ with $\eta=\left(\eta_{1}, \ldots, \eta_{n-1}\right)$ one has

$$
\left|\eta_{j}\right| \leq\left|\eta_{i_{0}}\right| \text { for any } j=1, \ldots, n-1 \text {. }
$$

For simplicity of the notation we will assume that $i_{0}=1$, i.e. $\left|\eta_{j}\right| \leq\left|\eta_{1}\right|$.

For $\xi=(\eta, \zeta) \in T$ let us consider the smooth map

$$
F_{\eta, \zeta, \varepsilon}: \mathbb{R}^{n-1} \rightarrow \mathbb{R}^{n-1}
$$

given by $F_{\eta, \zeta, \varepsilon}\left(v_{1}, v_{2}, \ldots, v_{n-1}\right):=\left(v_{1}+<\frac{\zeta}{\eta_{1}}, M(v, \varepsilon)>, v_{2}, \ldots, v_{n-1}\right)$.

Let us fix a large ball $B \subset \mathbb{R}^{n-1}$ containing the supports of all forms $\omega_{\varepsilon}, \varepsilon \in[0,1]$. Since for any $(\eta, \zeta) \in T$ one has $|\zeta| \leq C^{\prime}\left|\eta_{1}\right|$ for some constant $C^{\prime}$, and since $M(v, 0)=0$, it follows that there exists $\varepsilon_{0}>0$ such that for any $\varepsilon \in\left[0, \varepsilon_{0}\right]$ and any $(\eta, \zeta) \in T$ the map $F_{\eta, \zeta, \varepsilon}$ is a diffeomorphism of the ball $B$ onto its image. Moreover any $C^{k}$-norm of it and its inverse can be estimated by a constant independent of $(\eta, \zeta) \in T, \varepsilon \in\left[0, \varepsilon_{0}\right]$.

The expression in the exponent in (3.6.19) is

$$
\begin{gathered}
<\eta, v>+<\zeta, M(v, \varepsilon)>=\sum_{l=1}^{n-1} \eta_{l} v_{l}+<\zeta, M(v, \varepsilon)>= \\
\eta_{1} \cdot\left(v_{1}+<\frac{\zeta}{\eta_{1}} M(v, \varepsilon), v>\right)+\sum_{l=2}^{n-1} \eta_{l} v_{l}=<\eta, F_{\eta, \zeta, \varepsilon}(v)>.
\end{gathered}
$$

Hence after the change of variables using $F_{\eta, \zeta, \varepsilon}$, the inequality (3.6.19) becomes equivalent to

$$
\varlimsup_{\varepsilon \rightarrow+0}\left(\sup _{(\eta, \zeta) \in T}\left|\int_{\mathbb{R}^{n-1}} e^{i<\eta, v>} \cdot\left(F_{\eta, \zeta, \varepsilon}^{-1}\right)^{*} \omega_{\varepsilon}\right| \cdot\left(1+|\eta|^{N}\right)\right)<\infty .
$$

But for $(\eta, \zeta) \in T, \varepsilon \in\left[0, \varepsilon_{0}\right]$ the forms $\left(F_{\eta, \zeta, \varepsilon}^{-1}\right)^{*} \omega_{\varepsilon}$ have uniformly bounded supports, and all their $C^{k}$-norms are uniformly bounded. This readily implies (3.6.20). Thus (3.6.18) is proved. 
Similarly, but simpler, one proves that there exists a constant $C_{N}$ such that

$$
\sup _{\xi \in T}\left|\int_{N\left(A_{0}\right)} e^{i<\xi, y>} \omega(y)\right| \cdot\left(1+|\xi|^{N}\right)<C_{N}
$$

It remains to show that (3.6.21), (3.6.18) imply (3.6.17). To prove it, let us fix $\kappa>0$. There exist $\varepsilon_{1}>0$ and $C>0$ such that

$$
\sup _{\varepsilon \in\left[0, \varepsilon_{1}\right]} \sup _{\xi \in T}\left|\int_{N\left(A_{\varepsilon}\right)} e^{i<\xi, y>} \omega(y)\right| \cdot\left(1+|\xi|^{N+1}\right) \leq C .
$$

Then for $\xi \in T$ such that $|\xi|>\frac{2 C}{\kappa}$ we have for any $\varepsilon \in\left(0, \varepsilon_{1}\right)$

$$
\left|\int_{N\left(A_{\varepsilon}\right)} e^{i<\xi, y>} \omega(y)-\int_{N\left(A_{0}\right)} e^{i<\xi, y>} \omega(y)\right| \cdot\left(1+|\xi|^{N}\right) \leq \frac{2 C}{|\xi|}<\kappa .
$$

On the other hand for $|\xi| \leq \frac{2 C}{\kappa}$ we have

$$
\begin{array}{r}
\sup _{|\xi| \leq \frac{2 C}{\kappa}}\left|\int_{N\left(A_{\varepsilon}\right)} e^{i<\xi, y>} \omega(y)-\int_{N\left(A_{0}\right)} e^{i<\xi, y>} \omega(y)\right|= \\
\sup _{|\xi| \leq \frac{2 C}{\kappa}}\left|\int_{\mathbb{R}^{n-1}} e^{i<\eta, v>}\left(e^{i<\zeta, M(v, \varepsilon)>} \omega_{\varepsilon}(v)-\omega(v)\right)\right| \text { as } \varepsilon \rightarrow+0
\end{array}
$$

which follows from the facts that the expression under the integral tends to 0 uniformly in $\xi$ when $|\xi| \leq \frac{2 C}{\kappa}$. This implies (3.6.20). Hence Proposition 3.6.5 is proved. Q.E.D.

\section{Valuations and integral geometry.}

\subsection{General Radon transform on valuations.}

Let $X_{1} \stackrel{q_{1}}{\leftarrow} Z \stackrel{q_{2}}{\rightarrow} X_{2}$ be a double fibration. Then we have the map $i_{Z}:=q_{1} \times q_{2}$,

$$
i_{Z}: Z \rightarrow X_{1} \times X_{2}
$$

which is a closed imbedding by the definition of a double fibration. The goal of this section is to define a map

$$
\phi \mapsto q_{2 *}\left(\gamma \cdot q_{1}^{*} \phi\right)
$$

where $\gamma$ is a fixed smooth valuation on $Z$ ( $\gamma$ is called kernel), and $\phi$ is a valuation on $X_{1}$, either smooth or generalized. We will define such a map under appropriate assumptions on the classes of valuations considered (Theorem 4.1.1) or also on the double fibration (Theorem 4.1.4).

We will denote $X:=X_{1} \times X_{2}$. 
4.1.1 Theorem. Let $X_{1} \stackrel{q_{1}}{\leftarrow} Z \stackrel{q_{2}}{\longrightarrow} X_{2}$ be a double fibration such that $q_{2}$ is proper. Let $\gamma \in V^{\infty}(Z)$. Then the map $\phi \mapsto q_{2 *}\left(\gamma \cdot q_{1}^{*} \phi\right)$ is a well defined linear continuous map

$$
R_{\gamma}: V^{\infty}\left(X_{1}\right) \rightarrow V^{-\infty}\left(X_{2}\right) .
$$

First we will need a general lemma where we use the notation $\pi_{X *} \Gamma$ introduced in Section 1.4 after Remark 1.4.9.

4.1.2 Lemma. Let $X$ be a smooth manifold. Let $\Gamma \subset T^{*} \mathbb{P}_{X} \backslash \underline{0}$ be closed conic subset such that $\pi_{X *}(\Gamma)=\emptyset$. Then the product

$$
V_{\emptyset, \Gamma}^{-\infty}(X) \times V^{\infty}(X) \rightarrow V_{\emptyset, \Gamma}^{-\infty}(X)
$$

is a bilinear jointly sequentially continuous map.

Proof. In the proof we may and will assume that $X$ is a vector space in order to simplify the notation. Let $\phi \in V_{\emptyset, \Gamma}^{-\infty}(X), \psi \in V^{\infty}(X)$ be represented by pairs of currents $\left(C_{\phi}, T_{\phi}\right)$, $\left(C_{\psi}, T_{\psi}\right)$ respectively. Thus

$$
\begin{aligned}
& W F\left(C_{\phi}\right)=\emptyset, W F\left(T_{\phi}\right) \subset \Gamma, \\
& W F\left(C_{\psi}\right)=\emptyset, W F\left(T_{\psi}\right)=\emptyset .
\end{aligned}
$$

By (1.6.9)-(1.6.10) the product $\phi \cdot \psi$ is given by the pair $(C, T)$ where

$$
\begin{array}{r}
C=C_{\phi} \cap C_{\psi}, \\
T=(-1)^{n} \bar{p}_{*} \bar{\Phi}^{*}\left(q_{1}^{*} T_{\phi} \cap q_{2}^{*} T_{\psi}\right)+\pi_{X}^{*} C_{\phi} \cap T_{\psi}+T_{\phi} \cap \pi_{X}^{*} C_{\psi}
\end{array}
$$

where the maps

$$
\mathbb{P}_{X} \stackrel{\bar{p}}{\leftarrow} \overline{\mathbb{P}} \stackrel{\bar{\Phi}}{\rightarrow} \mathbb{P}_{X} \times_{X} \mathbb{P}_{X}
$$

defined in (1.6.1) and (1.6.2) of Section 1.6, and $q_{i}: \mathbb{P}_{X} \times_{X} \mathbb{P}_{X} \rightarrow \mathbb{P}_{X}, i=1,2$, be the natural projections onto the first and second factors respectively. Recall that $\overline{\mathbb{P}}$ is the oriented blow up of the space $\mathbb{P}_{X \times X} \cap \pi_{X \times X}^{-1}(\Delta)$ (where $\Delta \subset X \times X$ is the diagonal) along the submanifold $\mathcal{M}_{0} \sqcup \mathcal{M}_{1} \sqcup \mathcal{M}_{2}$ where

$$
\begin{aligned}
\mathcal{M}_{0} & :=\{(x,[\xi:-\xi])\}, \\
\mathcal{M}_{1} & :=\{(x,[\xi: 0])\}, \\
\mathcal{M}_{2} & :=\{(x,[0: \xi])\}
\end{aligned}
$$

We denote by $\mathcal{N}_{i}, i=0,1,2$, the preimages in $\overline{\mathbb{P}}$ of the sets $\mathcal{M}_{i}$.

It is easy to see that

$$
W F\left(q_{1}^{*} T_{\phi} \cap q_{2}^{*} T_{\psi}\right) \subset q_{1}^{*}(\Gamma) .
$$

Then in order to prove the lemma it suffices to show that for any $\phi \in V_{\emptyset, \Gamma}^{-\infty}(X), \psi \in V^{\infty}(X)$ one has

$$
\begin{array}{r}
W F\left(C_{\phi} \cap C_{\psi}\right)=\emptyset, \\
W F\left(T_{\phi} \cap \pi_{X}^{*} C_{\psi}\right) \subset \Gamma, \\
W F\left(\pi_{X}^{*} C_{\phi} \cap T_{\psi}\right) \subset \Gamma, \\
\bar{p}_{*} \bar{\Phi}^{*}\left(q_{1}^{*} \Gamma\right) \subset \Gamma
\end{array}
$$


where the notations of pull-back and push-forward of sets were introduced in Section 1.4 in Proposition 1.4 .4 and after Remark 1.4 .9 respectively.

The inclusions (4.1.1)-(4.1.3) are obvious. It remains to check (4.1.4) which can be rewritten as

$$
\bar{p}_{*}\left(q_{1} \circ \bar{\Phi}\right)^{*}(\Gamma) \subset \Gamma
$$

Let us fix points

$$
X_{0}=\left(x_{0},\left[\xi_{0}\right]\right) \in \mathbb{P}_{X}, X_{1}=\left(x_{1},\left[\xi_{1}\right]\right) \in \mathbb{P}_{X}, \alpha \in \overline{\mathbb{P}}
$$

such that

$$
\bar{p}(\alpha)=X_{0},\left(q_{1} \circ \bar{\Phi}\right)(\alpha)=X_{1} .
$$

then necessarily $x_{0}=x_{1}$. We have to show that if $v \in T_{X_{0}}^{*} \mathbb{P}_{X} \backslash\{0\}$ and $\left.w \in \Gamma\right|_{X_{1}}$ satisfy

$$
d \bar{p}^{*}(v)=d\left(q_{1} \circ \bar{\Phi}\right)^{*}(w)
$$

then $v \in \Gamma$ (here the differentials are taken at the point $\alpha \in \overline{\mathbb{P}}$ ).

We will consider 4 cases:

Case 1: $\alpha \notin \mathcal{N}_{0} \bigsqcup \mathcal{N}_{1} \bigsqcup \mathcal{N}_{2}$;

Case 2: $\alpha \in \mathcal{N}_{0}$;

Case 3: $\alpha \in \mathcal{N}_{1}$

Case 4: $\alpha \in \mathcal{N}_{2}$.

Before we will start considering these cases let us introduce more notation. Since we assume that $X$ is a vector space, we can write $\mathbb{P}_{X}=X \times \mathbb{P}_{+}\left(X^{*}\right)$, and $\overline{\mathbb{P}}=X \times Y$ where $Y$ is an appropriate blow up space of $\mathbb{P}_{+}\left(X^{*} \oplus X^{*}\right)$. The maps $\bar{p}$ and $q_{1} \circ \bar{\Phi}$ preserve these decompositions, more precisely $\bar{p}=\left(I d_{X}, \tilde{p}\right)$ where $\tilde{p}: Y \rightarrow \mathbb{P}_{+}\left(X^{*}\right)$, and $q_{1} \circ \bar{\Phi}=\left(I d_{X}, \tilde{\Phi}\right)$ where $\tilde{\Phi}: Y \rightarrow \mathbb{P}_{+}\left(X^{*}\right)$. It is easy to see that $\tilde{p}$ and $\tilde{\Phi}$ are submersions as well as their restrictions to the boundary of $Y$. Let us write the vector $v \in T_{X_{1}}^{*} \mathbb{P}_{X}$ in the form $v=\left(v^{\prime}, v^{\prime \prime}\right)$ where $v^{\prime} \in T_{x_{0}}^{*} X, v^{\prime \prime} \in T_{\left[\xi_{0}\right]}^{*} \mathbb{P}_{+}\left(X^{*}\right)$. Let us show that if (4.1.6) is satisfied then

$$
v^{\prime \prime} \neq 0
$$

Indeed otherwise $d \bar{p}^{*}(v)=\left(v^{\prime}, 0\right)$. Hence $d\left(q_{1} \circ \bar{\Phi}\right)^{*}(w)=\left(v^{\prime}, 0\right)$. But since $\tilde{\Phi}$ is a submersion $w$ has the form $w=\left(v^{\prime}, 0\right)$. This implies that $v^{\prime} \in \pi_{X *}(\Gamma)=\emptyset$. This is a contradiction.

Now let us consider Case 1. Thus $\alpha \notin \mathcal{N}_{0} \sqcup \mathcal{N}_{1} \sqcup \mathcal{N}_{2}$. We may assume that $\alpha \in\left(\mathbb{P}_{X} \times_{X}\right.$ $\left.\mathbb{P}_{X}\right) \backslash\left(\mathcal{M}_{0} \sqcup \mathcal{M}_{1} \sqcup \mathcal{M}_{2}\right)$. Then $\alpha$ must have the form

$$
\alpha=\left(x_{0},\left[\xi_{1}: \eta\right]\right) \text { with }[\eta] \neq\left[-\xi_{1}\right] \text { and }\left[\xi_{1}+\eta\right]=\left[\xi_{0}\right] .
$$

Let us consider the subspace $S \subset T_{\alpha} \overline{\mathbb{P}}$ defined as the tangent space at $\alpha$ to the submanifold $\left\{\left(x,\left[\xi_{1}: \zeta\right]\right) \mid x \in X, \zeta \in X^{*}\right\}$ (here $\xi_{1}$ is fixed). Let us denote also by $S^{\prime}$ the tangent space to $\left\{\left[\xi_{1}: \zeta\right] \mid \zeta \in X^{*}\right\}$ at $\left[\xi_{1}: \eta\right]$. In this notation we have the following decompositions:

$$
\begin{array}{r}
T_{X_{0}} \mathbb{P}_{X}=X \oplus T_{\left[\xi_{0}\right]}\left(\mathbb{P}_{+}\left(X^{*}\right)\right), \\
T_{X_{1}} \mathbb{P}_{X}=X \oplus T_{\left[\xi_{1}\right]}\left(\mathbb{P}_{+}\left(X^{*}\right)\right), \\
S=X \oplus S^{\prime} .
\end{array}
$$


It is clear that the restriction of the differential $\left.d \bar{p}\right|_{S}: S \rightarrow T_{X_{0}} \mathbb{P}_{X}$ is an epimorphism, and moreover $\left.d \bar{p}\right|_{S}$ maps the decomposition (4.1.10) to the decomposition (4.1.8).

Next the restriction of the differential $\left.d\left(q_{1} \circ \bar{\Phi}\right)\right|_{S}: S \rightarrow T_{X_{1}} \mathbb{P}_{X}$ maps decomposition (4.1.10) to (4.1.9), and its kernel is equal to $S^{\prime}$. Hence for $\left.w \in \Gamma\right|_{X_{1}}$ one has $\left.d\left(q_{1} \circ \bar{\Phi}\right)^{*}(w)\right|_{S} \in$ $X^{*} \times\left\{0_{S^{*}}\right\}$. This implies that if $d \bar{p}^{*}(v)=d\left(q_{1} \circ \bar{\Phi}\right)^{*}(w)$ then

$$
v=\left(v^{\prime}, 0\right) \in X^{*} \times\{0\} \subset T_{X_{0}}^{*} \mathbb{P}_{X}
$$

(here we have used that $\left.d \bar{p}\right|_{S}: S \rightarrow T_{X_{0}} \mathbb{P}_{X}$ is an epimorphism). This contradicts to (4.1.7), thus Case 1 is proved.

Let us consider Case 2. Thus we assume that $\alpha \in \mathcal{N}_{0}$. It is easy to see that there exists a subspace $S_{0} \subset T_{\alpha} \overline{\mathbb{P}}$ of the form $S_{0}=X \oplus S_{0}^{\prime}$ with $S_{0}^{\prime} \subset T Y$, such that $\left.d \tilde{p}\right|_{S_{0}^{\prime}}: S_{0}^{\prime}$ $\rightarrow T_{x_{0}} \mathbb{P}_{+}\left(X^{*}\right)$ is an isomorphism, and $\left.d \tilde{\Phi}\right|_{S_{0}^{\prime}}: S_{0}^{\prime} \rightarrow T_{x_{1}}\left(\mathbb{P}_{+}\left(X^{*}\right)\right)=T_{x_{0}}\left(\mathbb{P}_{+}\left(X^{*}\right)\right)$ vanishes. The second fact implies that $\left.d\left(q_{1} \circ \bar{\Phi}\right)^{*}\right|_{S^{\prime}}(w)$ has the form $\left(w^{\prime}, 0_{S^{\prime}}\right)$ with $w^{\prime} \in X^{*}$. This and the first fact imply that $v=\left(w^{\prime}, 0\right)$. This is again a contradiction to (4.1.7), and Case 2 is proved.

Let us consider Case 3. Namely assume that $\alpha \in \mathcal{N}_{1}$. This implies that $\left[\xi_{0}\right]=\left[\xi_{1}\right]$. Hence $X_{0}=X_{1}$. It is easy to see that there exists a linear subspace $S_{1} \subset T_{\alpha} \overline{\mathbb{P}}$ of the form $S_{1}=X \oplus S_{1}^{\prime}$ with $S_{1}^{\prime} \subset T Y$ such that $\left.d \tilde{p}\right|_{S_{1}^{\prime}}: S_{1}^{\prime} \rightarrow T_{\left[\xi_{0}\right]} \mathbb{P}_{+}\left(X^{*}\right)$ and $\left.d \tilde{\Phi}\right|_{S_{1}^{\prime}}: S_{1}^{\prime} \rightarrow T_{\left[\xi_{0}\right]} \mathbb{P}_{+}\left(X^{*}\right)$ are isomorphisms, and

$$
\left.d \tilde{\Phi}\right|_{S_{1}^{\prime}} \circ\left(\left.d \tilde{p}\right|_{S_{1}^{\prime}}\right)^{-1}: T_{\left[\xi_{0}\right]} \mathbb{P}_{+}\left(X^{*}\right) \rightarrow T_{\left[\xi_{0}\right]} \mathbb{P}_{+}\left(X^{*}\right)
$$

is the identity map. This immediately implies that if $d \bar{p}^{*}(v)=d\left(q_{1} \circ \bar{\Phi}\right)^{*}(w)$ then $v=w$. Hence $v \in \Gamma$.

Let us consider Case 4, i.e. $\alpha \in \mathcal{N}_{2}$. It is easy to see that there exists a subspace $S_{2} \subset T_{\alpha} \overline{\mathbb{P}}$ of the form $S_{2}=X \oplus S_{2}^{\prime}$ with $S_{2}^{\prime} \subset T Y$ such that $\left.d \tilde{p}\right|_{S_{2}^{\prime}}: S_{2}^{\prime} \rightarrow T_{x_{0}}\left(\mathbb{P}_{+}\left(X^{*}\right)\right)$ vanishes, and $\left.d \tilde{\Phi}\right|_{S_{2}^{\prime}}: S_{2}^{\prime} \rightarrow T_{x_{0}}\left(\mathbb{P}_{+}\left(X^{*}\right)\right)$ is an isomorphism. These two facts and the assumption $d \bar{p}^{*}(v)=$ $d\left(q_{1} \circ \bar{\Phi}\right)^{*}(w)$ imply that $w=\left(w^{\prime}, 0\right)$. Hence $w^{\prime} \in \pi_{X *}(\Gamma)=\emptyset$ which is a contradiction.

Lemma is proved. Q.E.D.

Proof of Theorem 4.1.1. For $i=1,2$ let us denote for brevity

$$
\Gamma_{i}:=T_{Z \times_{X_{i}} \mathbb{P}_{X_{i}}}^{*}(Z) \backslash \underline{0} .
$$

Obviously $\pi_{X_{i} *}\left(\Gamma_{i}\right)=\emptyset$. The map of pull-back

$$
q_{i}^{*}: V^{\infty}\left(X_{i}\right) \rightarrow V_{\emptyset, \Gamma_{i}}^{-\infty}(Z)
$$

is a continuous linear operator (since $V^{\infty}\left(X_{1}\right)$ is a Fréchet space, sequential continuity implies topological continuity). By this and Lemma 4.1.2 the map $\left[\phi \mapsto \gamma \cdot q_{1}^{*} \phi\right]$ is a continuous linear operator $V^{\infty}\left(X_{1}\right) \rightarrow V_{\emptyset, \Gamma_{1}}^{-\infty}(Z)$. Thus in order to finish the proof of the theorem it remains to show that the push-forward map $q_{2 *}: V^{-\infty} \emptyset, \Gamma_{1}(Z) \rightarrow V^{-\infty}\left(X_{2}\right)$ is well defined in the sense 
of Section 3.6, then it is automatically sequentially continuous. For this it suffices to check that the pairs of sets $\left(\emptyset, \Gamma_{1}\right)$ and $\left(\emptyset, \Gamma_{2}\right)$ satisfy the conditions (3.6.1)-(3.6.5). The conditions (3.6.1)-(3.6.4) are satisfied trivially in this case.

Let us check (3.6.5). It is easy to see that this condition is equivalent the following general claim.

4.1.3 Claim. If $X_{1} \stackrel{q_{1}}{\leftarrow} Z \stackrel{q_{2}}{\longrightarrow} X_{2}$ is a double fibration, then the sets $Z \times_{X_{1}} \mathbb{P}_{X_{1}}$ and $Z \times_{X_{2}} \mathbb{P}_{X_{2}}$ intersect transversally in $\mathbb{P}_{Z}$.

Let us prove the claim. It is equivalent that the sets $M_{1}:=Z \times_{X_{1}}\left(T^{*} X_{1} \backslash \underline{0}\right)$ and $M_{2}:=$ $Z \times_{X_{2}}\left(T^{*} X_{2} \backslash \underline{0}\right)$ intersect transversally in $T^{*} Z$.

Let us fix a point $A \in M_{1} \cap M_{2}$. We have to show that $T_{A} M_{1}+T_{A} M_{2}=T_{A}\left(T^{*} Z\right)$. Let us denote by $F$ the only fiber of the natural projection $T^{*} Z \rightarrow Z$ which contains $A$. Since $q_{1}, q_{2}$ are submersions we have

$$
T_{A} F+T_{A} M_{i}=T_{A}\left(T^{*} Z\right), i=1,2 .
$$

On the other hand since $q_{1} \times q_{2}: Z \rightarrow X_{1} \times X_{2}$ is an imbedding

$$
T_{A} F \subset T_{A} M_{1}+T_{A} M_{2}
$$

From (4.1.12) and (4.1.13) we conclude that $T_{A} M_{1}+T_{A} M_{2}=T_{A}\left(T^{*} Z\right)$. Hence $M_{1}, M_{2}$ are transversal and Claim 4.1.3 is proved.

Hence the condition (3.6.5) is proved, and Theorem 4.1.1 as well. Q.E.D.

In order to formulate the second main result let us introduce more notation. For $j=1,2$ let

$$
p_{j}: Z \times_{X_{j}} \mathbb{P}_{X_{j}} \rightarrow \mathbb{P}_{X_{j}}, i_{j}: Z \times_{X_{j}} \mathbb{P}_{X_{j}} \hookrightarrow \mathbb{P}_{Z}
$$

be the obvious maps.

4.1.4 Theorem. Let $X_{1} \stackrel{q_{1}}{\leftarrow} Z \stackrel{q_{2}}{\longrightarrow} X_{2}$ be a double fibration. Assume in addition that $T_{Z \times_{X_{1}} \mathbb{P}_{X_{1}}} \mathbb{P}_{Z}$ and $i_{2 *} p_{2}^{*}\left(T^{*} \mathbb{P}_{X_{2}} \backslash \underline{0}\right)$ are disjoint subsets of $T^{*} \mathbb{P}_{Z}$. Let $\gamma \in V^{\infty}(Z)$.

(1) If $q_{1}$ is proper then the map $V^{\infty}\left(X_{2}\right) \rightarrow V^{-\infty}\left(X_{1}\right)$ defined in Theorem 4.1.1 by $\left[\psi \mapsto q_{1 *}\left(\gamma \cdot q_{2}^{*} \psi\right)\right]$ extends (uniquely) to a continuous linear operator

$$
V^{-\infty}\left(X_{2}\right) \rightarrow V^{-\infty}\left(X_{1}\right)
$$

when both spaces are equipped with the weak topologies.

(2) If $q_{2}$ is proper then the map $\left[\phi \mapsto q_{2 *}\left(\gamma \cdot q_{1}^{*} \phi\right)\right]$ is a well defined continuous linear operator

$$
V^{\infty}\left(X_{1}\right) \rightarrow V^{\infty}\left(X_{2}\right)
$$

when both spaces are equipped with their usual Fréchet topologies.

4.1.5 Remark. Below in Proposition 4.1.6 we will show that the assumptions of Theorem 4.1 .4 are satisfied provided that the natural projection $T_{Z}^{*}\left(X_{1} \times X_{2}\right) \backslash\{0\} \rightarrow T^{*} X_{2} \backslash\{0\}$ is a submersion. 
Proof of Theorem 4.1.4. Let us start with part (2). By Theorem 4.1.1 we have a separately continuous bilinear map $V^{\infty}\left(X_{1}\right) \times V_{c}^{\infty}\left(X_{2}\right) \rightarrow \mathbb{C}$ given by

$$
(\phi, \psi) \mapsto \int_{Z}\left(q_{1}^{*} \phi \cdot \gamma\right) \cdot q_{2}^{*} \psi .
$$

First let us show that the last map extends (uniquely, of course) to a separately continuous bilinear map

$$
V^{\infty}\left(X_{1}\right) \times V_{c}^{-\infty}\left(X_{2}\right) \rightarrow \mathbb{C} .
$$

Let us denote for brevity

$$
\Lambda:=q_{2}^{*}\left(T^{*} X_{2} \backslash \underline{0}\right), \Gamma:=i_{2 *} p_{2}^{*}\left(T^{*} \mathbb{P}_{X_{2}} \backslash \underline{0}\right) .
$$

Also as above in this section we denote $\Gamma_{j}:=\left(T_{Z \times_{X_{j}} \mathbb{P}_{X_{j}}} \mathbb{P}_{Z}\right) \backslash \underline{0}$. In this notation for any $\psi \in V^{-\infty}\left(X_{2}\right)$ we have $q_{2}^{*} \psi \in V_{\Lambda, \Gamma}^{-\infty}(Z)$. Also it is clear that $\pi_{Z *}\left(\Gamma_{j}\right)=0$. Hence by Lemma 4.1.2 the map $V^{\infty}\left(X_{1}\right) \rightarrow V_{\emptyset, \Gamma_{1}}^{-\infty}(Z)$ given by $\phi \mapsto \gamma \cdot q_{1}^{*} \phi$ is continuous.

Thus it suffices to show that the pairs of sets $(\Lambda, \Gamma)$ and $\left(\emptyset, \Gamma_{1}\right)$ satisfy the conditions (3.6.1)-(3.6.5). Explicitly in this case they mean:

$$
\begin{array}{r}
\Lambda \cap s(\emptyset)=\emptyset, \\
\Gamma \cap s\left(\pi_{Z}^{*} \emptyset\right)=\emptyset, \\
\Gamma_{1} \cap s\left(\pi_{Z}^{*} \Lambda\right)=\emptyset, \\
\text { if }\left(z,\left[\xi_{1}\right], u_{1}, 0\right) \in \Gamma_{1} \text { and }(z,[\xi], u, 0) \in \Gamma \text { then } u_{1} \neq-u, \\
\text { if }(z,[\xi]) \in \mathbb{P}_{Z},\left.\left(u, \eta_{1}\right) \in \Gamma_{1}\right|_{(z,[\xi])}, \text { and }\left.(-u, \eta) \in \Gamma\right|_{(z,[-\xi]), \text { then }} \\
d \theta^{*}\left(0, \eta_{1}, \eta\right) \neq(0, l,-l) \in T_{(z,[\xi],[\xi])}^{*}\left(\mathbb{P}_{Z} \times{ }_{Z} \mathbb{P}_{Z}\right)
\end{array}
$$

where $\theta: \mathbb{P}_{Z} \times_{Z} \mathbb{P}_{Z} \rightarrow \mathbb{P}_{Z} \times_{Z} \mathbb{P}_{Z}$ is defined by $\theta\left(z,\left[\zeta_{1}\right],\left[\zeta_{2}\right]\right)=\left(z,\left[\zeta_{1}\right],\left[-\zeta_{2}\right]\right)$.

The conditions (4.1.14), (4.1.15) are satisfied trivially. The conditions (4.1.16), (4.1.17) are satisfied since $\Gamma_{1}$ contains no elements of the form $\left(z,\left[\xi_{1}\right], u_{1}, 0\right)$. The condition (4.1.18) easily follows from our assumption $\Gamma_{1} \cap \Gamma=\emptyset$.

Thus we got a jointly sequentially continuous bilinear map

$$
B: V^{\infty}\left(X_{1}\right) \times V_{c}^{-\infty}\left(X_{2}\right) \rightarrow \mathbb{C} .
$$

Then for any $\phi \in V^{\infty}\left(X_{1}\right)$ the map $V_{c}^{-\infty}\left(X_{2}\right) \rightarrow \mathbb{C}$ given by $\psi \mapsto B(\phi, \psi)$ is sequentially continuous. But the topology on $V_{c}^{-\infty}\left(X_{2}\right)=\left(V^{\infty}\left(X_{2}\right)\right)^{*}$ coincides with the weak topology. Since $V^{\infty}\left(X_{2}\right)$ separable Fréchet space, any sequentially continuous (in the weak topology) linear functional on its dual $\left(V^{\infty}\left(X_{2}\right)\right)^{*}$ is continuous in the weak topology (by Ch. IV, $\S 6$, Corollary 3 in [36]). This implies that $B$ is separately continuous.

Then by duality $B$ induces a continuous linear map $V^{\infty}\left(X_{1}\right) \rightarrow V^{\infty}\left(X_{2}\right)$ where the target space is equipped with the weak topology. It remains to prove the continuity of this map when the target space is equipped with the usual Fréchet topology. Let us denote temporarily this map by $R$. Since $V^{\infty}\left(X_{1}\right)$ and $V^{\infty}\left(X_{2}\right)$ are Fréchet spaces, by the closed graph theorem (see e.g. [33], Ch. VI, §3, Thm. 8) it suffices to show that $R$ has a closed graph. Assume 
that a sequence $\phi_{N} \rightarrow \phi$ in $V^{\infty}\left(X_{1}\right)$, and $R\left(\phi_{N}\right) \rightarrow \psi$ in $V^{\infty}\left(X_{2}\right)$. Since $R$ is continuous is the weak topology, it follows that $\psi=R(\phi)$. Hence the graph of $R$ is closed in the Fréchet topology. Part (2) is proved.

Let us prove now part (1). By Theorem 4.1.1 we have a separately continuous bilinear map

$$
V_{c}^{\infty}\left(X_{1}\right) \times V^{\infty}\left(X_{2}\right) \rightarrow \mathbb{C}
$$

given by $(\phi, \psi) \mapsto \int_{Z} q_{1}^{*} \phi \cdot\left(\gamma \cdot q_{2}^{*} \psi\right)$. Let us show that this map extends (uniquely) to a separately continuous bilinear map

$$
V_{c}^{\infty}\left(X_{1}\right) \times V^{-\infty}\left(X_{2}\right) \rightarrow \mathbb{C} .
$$

First observe that if $\phi, \psi$ are smooth then $q_{1}^{*} \phi \in V_{\emptyset, \Gamma_{1}}^{-\infty}(Z), q_{2}^{*} \psi \in V_{\emptyset, \Gamma_{2}}^{-\infty}(Z)$, and by Lemma 4.1 .2

$$
q_{1}^{*} \phi \cdot \gamma \in V_{\emptyset, \Gamma_{1}}^{-\infty}(Z), \gamma \cdot q_{2}^{*} \psi \in V_{\emptyset, \Gamma_{2}}^{-\infty}(Z) .
$$

Hence for smooth $\phi, \psi$ the products $\left(q_{1}^{*} \phi \cdot \gamma\right) \cdot q_{2}^{*} \psi$ and $q_{1}^{*} \phi \cdot\left(\gamma \cdot q_{2}^{*} \psi\right)$ are defined and are equal to each other by the associativity proven in [11], Theorem 8.3. In particular

$$
\int_{Z} q_{1}^{*} \phi \cdot\left(\gamma \cdot q_{2}^{*} \psi\right)=\int_{Z}\left(q_{1}^{*} \phi \cdot \gamma\right) \cdot q_{2}^{*} \psi
$$

Let us show that the right hand side of (4.1.19) extends to a jointly sequentially continuous bilinear map $V_{c}^{\infty}\left(X_{1}\right) \times V^{-\infty}\left(X_{2}\right) \rightarrow \mathbb{C}$. To prove it, it suffices to show that the pairs of sets $(\Lambda, \Gamma)$ and $\left(\emptyset, \Gamma_{2}\right)$ satisfy the conditions (3.6.1)-(3.6.5). But this has been proven in the proof of part (2) of the theorem. Next, as in the proof of part (2), this bilinear functional separately continuous since $V^{-\infty}\left(X_{2}\right)=\left(V_{c}^{\infty}\left(X_{2}\right)\right)^{*}$ and any sequentially continuous (in the weak topology) linear functional on it is continuous. Q.E.D.

Now we would like to give a sufficient condition on a double fibration implying the assumption of Theorem 4.1.4. Recall that $Z \subset X_{1} \times X_{2}$ is a closed submanifold. Consider the conormal bundle $T_{Z}^{*}\left(X_{1} \times X_{2}\right) \subset T^{*} X_{1} \times T^{*} X_{2}$. Since $q_{i}$ 's are submersions, the projections $T_{Z}^{*}\left(X_{1} \times X_{2}\right) \rightarrow T^{*} X_{i}, i=1,2$, map $T_{Z}^{*}\left(X_{1} \times X_{2}\right) \backslash \underline{0}$ to $T^{*} X_{i} \backslash \underline{0}$. We are going to prove

4.1.6 Proposition. Assume that the double fibration $X_{1} \stackrel{q_{1}}{\leftarrow} Z \stackrel{q_{2}}{\longrightarrow} X_{2}$ satisfies that

$$
T_{Z}^{*}\left(X_{1} \times X_{2}\right) \backslash \underline{0} \rightarrow T^{*} X_{2} \backslash \underline{0}
$$

is a submersion. Then the assumption of Theorem 4.1.4 is satisfied, namely

$$
\left(T_{Z \times_{X_{1}} \mathbb{P}_{X_{1}}}^{*} \mathbb{P}_{Z}\right) \cap i_{2 *} p_{2}^{*}\left(T^{*} \mathbb{P}_{X_{2}} \backslash \underline{0}\right)=\emptyset .
$$

Proof. The $\operatorname{map} T_{Z}^{*}\left(X_{1} \times X_{2}\right) \backslash \underline{0} \rightarrow T^{*} X_{2} \backslash \underline{0}$ is a submersion if and only if the induced map

$$
\mathbb{P}_{+}\left(T_{Z}^{*}\left(X_{1} \times X_{2}\right)\right) \rightarrow \mathbb{P}_{X_{2}}
$$

is a submersion. 
By Claim 4.1.3 the submanifolds $Z \times_{X_{1}} \mathbb{P}_{X_{1}}$ and $Z \times_{X_{2}} \mathbb{P}_{X_{2}}$ intersect transversally in $\mathbb{P}_{Z}$. Let us denote their intersection by $\mathcal{C}$. We claim that the restriction of the obvious projection $p_{2}: Z \times_{X_{2}} \mathbb{P}_{X_{2}} \rightarrow \mathbb{P}_{X_{2}}$ to $\mathcal{C}$ is a submersion. For consider the map

$$
J: \mathbb{P}_{+}\left(T_{Z}^{*}\left(X_{1} \times X_{2}\right)\right) \rightarrow Z \times_{X_{2}} \mathbb{P}_{X_{2}}
$$

given by $J\left(z,\left[\xi_{1}: \xi_{2}\right]\right):=\left(z,\left[\xi_{2}\right]\right)$. It is easy to see that the image of $J$ is equal to $\mathcal{C}$, and $J$ is a diffeomorphism

$$
J: \mathbb{P}_{+}\left(T_{Z}^{*}\left(X_{1} \times X_{2}\right)\right) \stackrel{\sim}{\rightarrow} \mathcal{C}
$$

Moreover the composition

$$
\mathbb{P}_{+}\left(T_{Z}^{*}\left(X_{1} \times X_{2}\right)\right) \stackrel{J}{\rightarrow} Z \times_{X_{2}} \mathbb{P}_{X_{2}} \stackrel{p_{2}}{\rightarrow} \mathbb{P}_{X_{2}}
$$

coincides with the map (4.1.20) which, by the assumption, is a submersion. This implies that the restriction of $p_{2}$ to $\mathcal{C}$

$$
\left.p_{2}\right|_{\mathcal{C}}: \mathcal{C} \rightarrow \mathbb{P}_{X_{2}}
$$

is a submersion as we wanted.

Let us fix now a point $A \in \mathcal{C}$. Let us denote

$$
\begin{array}{r}
E:=T_{A} \mathbb{P}_{Z}, \\
L_{i}:=T_{A}\left(Z \times_{X_{i}} \mathbb{P}_{X_{i}}\right), i=1,2 .
\end{array}
$$

Since $Z \times_{X_{1}} \mathbb{P}_{X_{1}}$ and $Z \times_{X_{2}} \mathbb{P}_{X_{2}}$ are transversal, one has

$$
L_{1}+L_{2}=E \text {. }
$$

Obviously the fiber of $T_{Z \times_{X_{1}}}^{*} \mathbb{P}_{X_{1}} \mathbb{P}_{Z}$ over $A$ is

$$
\left.\left(T_{Z \times_{X_{1}} \mathbb{P}_{X_{1}}} \mathbb{P}_{Z}\right)\right|_{A}=L_{1}^{\perp} \subset E^{*} .
$$

It is easy to see that the fiber of $i_{2 *} p_{2}^{*}\left(T^{*} \mathbb{P}_{X_{2}}\right)$ over $A$ is equal to

$$
\left(\operatorname{Ker}\left(d p_{2}: L_{2} \rightarrow T_{p_{2}(A)} \mathbb{P}_{X_{2}}\right)\right)^{\perp} \text {. }
$$

Thus to prove the proposition we have to check that

$$
L_{1}+\operatorname{Ker}\left(d p_{2}: L_{2} \rightarrow T_{p_{2}(A)} \mathbb{P}_{X_{2}}\right)=E .
$$

Since, as we have proven, $\left.p_{2}\right|_{\mathcal{C}}: \mathcal{C} \rightarrow \mathbb{P}_{X_{2}}$ is a submersion, and obviously $T_{A} \mathcal{C}=L_{1} \cap L_{2}$, the map

$$
d p_{2}: L_{1} \cap L_{2} \rightarrow T_{p_{2}(A)} \mathbb{P}_{X_{2}}
$$

is onto. To prove (4.1.22) let us fix an arbitrary element $x \in E$. By (4.1.21) there exists a presentation $x=l_{1}+l_{2}$ with $l_{i} \in L_{i}$. By the surjectivity of $\left.d p_{2}\right|_{L_{1} \cap L_{2}}$, there exists $\tau \in L_{1} \cap L_{2}$ such that

$$
d p_{2}\left(l_{2}\right)=d p_{2}(\tau)
$$


Set $k:=l_{2}-\tau \in \operatorname{Ker}\left(\left.d p_{2}\right|_{L_{2}}\right)$. We have

$$
x=\left(l_{1}+\tau\right)+k \in L_{1}+\operatorname{Ker}\left(\left.d p_{2}\right|_{L_{2}}\right) .
$$

Thus (4.1.22) is proved. Hence the proposition is proved. Q.E.D.

Theorem 4.1.4 and Proposition 4.1.6 imply immediately the following corollary which we would like to state separately since it will be used in this form in the applications.

4.1.7 Corollary. Let $X_{1} \stackrel{q_{1}}{\leftarrow} Z \stackrel{q_{2}}{\longrightarrow} X_{2}$ be a double fibration. Assume that the projection $T_{Z}^{*}\left(X_{1} \times X_{2}\right) \backslash \underline{0} \rightarrow T^{*} X_{2} \backslash \underline{0}$ is a submersion.

(1) If $q_{1}$ is proper then the map $V^{\infty}\left(X_{2}\right) \rightarrow V^{-\infty}\left(X_{1}\right)$ defined by Theorem 4.1.1 as $\left[\psi \mapsto q_{1 *}\left(\gamma \cdot q_{2}^{*} \psi\right)\right]$ extends (uniquely) to a topologically continuous linear operator

$$
V^{-\infty}\left(X_{2}\right) \rightarrow V^{-\infty}\left(X_{1}\right)
$$

when both spaces are equipped with the weak topologies.

(2) If $q_{2}$ is proper then the map $\left[\phi \mapsto q_{2 *}\left(\gamma \cdot q_{1}^{*} \phi\right)\right]$ is a well defined topologically continuous linear operator

$$
V^{\infty}\left(X_{1}\right) \rightarrow V^{\infty}\left(X_{2}\right)
$$

when both spaces are equipped with their usual Fréchet topologies.

4.1.8 Example. Let us give some examples of double fibrations satisfying the assumptions of Corollary 4.1.7.

(1) Let $X=\mathbb{R}^{n}$. Let $Y=\mathcal{A} G r_{k, n}$ be the Grassmannian of affine $k$-dimensional subspaces in $\mathbb{R}^{n}, 0<k<n$. Let $Z$ be the incidence variety, i.e.

$$
Z:=\left\{(x, H) \in \mathbb{R}^{n} \times \mathcal{A} G r_{k, n} \mid x \in H\right\}
$$

Then $X=\mathbb{R}^{n} \stackrel{q_{1}}{\leftarrow} Z \stackrel{q_{2}}{\longrightarrow} \mathcal{A} G r_{k, n}=Y$ with the obvious projections $q_{1}, q_{2}$ is a double fibration, and the map

$$
T_{Z}^{*}(X \times Y) \backslash \underline{0} \rightarrow T^{*} X \backslash \underline{0}
$$

is a submersion.

(2) The next example will be particularly important in Section 4.3. Let $X=\mathbb{R P}^{n}$ be the real projective space, i.e. the space of real lines in $\mathbb{R}^{n+1}$ passing through 0 . Let $Y=\mathbb{R} \mathbb{P}^{n \vee}$ be the dual projective space, i.e. the space of real hyperplanes in $\mathbb{R}^{n+1}$ passing through 0 . Let $Z:=\left\{(l, H) \in \mathbb{R} \mathbb{P}^{n} \times \mathbb{R P}^{n+1} \mid l \subset H\right\}$ be again the incidence variety. Then $X=\mathbb{R} \mathbb{P}^{n} \stackrel{q_{1}}{\leftarrow} Z \stackrel{q_{2}}{\longrightarrow} \mathbb{R P}^{n \vee}=Y$ is a double fibration, and both projections

$$
T_{Z}^{*}(X \times Y) \backslash \underline{0} \rightarrow T^{*} X \backslash \underline{0}, T_{Z}^{*}(X \times Y) \backslash \underline{0} \rightarrow T^{*} Y \backslash \underline{0}
$$

are submersions. We will prove in Appendix that this double fibration satisfies the assumptions of Corollary 4.1.7.

(3) The example (2) can be generalized as follows. Let $\mathbb{K}$ be either $\mathbb{R}, \mathbb{C}$, or $\mathbb{H}$ (where $\mathbb{H}$ denotes the non-commutative field of quaternions). Fix natural numbers $k, m, n$ such that $0<k \neq m<n$. Denote by ${ }^{\mathbb{K}} G r_{k, n}$ the Grassmannian of $\mathbb{K}$-linear subspaces in $\mathbb{K}^{n}$ of 
$\mathbb{K}$-dimension $k$. Let $X={ }^{\mathbb{K}} G r_{k, n}, Y={ }^{\mathbb{K}} G r_{m, n}$. Let $Z$ be the incidence variety again. Then $X \stackrel{q_{1}}{\leftarrow} Z \stackrel{q_{2}}{\rightarrow} Y$ is a double fibration. If $k=1$ or $n-1$ then the projection

$$
T_{Z}^{*}(X \times Y) \backslash \underline{0} \rightarrow T^{*} X \backslash \underline{0} .
$$

is a submersion. We refer to Appendix for the proof of this fact.

(4) Let $X$ (resp. $Y$ ) be the Grassmannian of affine $\mathbb{K}$-subspaces in $\mathbb{K}^{n}$ of $\mathbb{K}$-dimension $k$ (resp. $m$ ). Assume that $k=1$ or $n-1$, and $k \neq m, 1<m<n$. Let $Z$ be the incidence variety. In this case all the properties of the corresponding double fibration remain the same as in the previous examples.

\subsection{Relations to the Gelfand style integral geometry.}

In this section we describe the explicit relation between the Radon transform on valuations introduced in Section 4.1 and the classical integral geometry in the spirit of Gelfand's school [20].

In a rather general setting, the Gelfand style integral geometry can be described as follows. For brevity we will denote by $\mathcal{M}^{\infty}(X)$ the space of smooth densities (i.e. smooth measures) on a manifold $X$. Let

$$
X_{1} \stackrel{q_{1}}{\leftarrow} Z \stackrel{q_{2}}{\rightarrow} X_{2}
$$

be a double fibration. Assume that $q_{2}$ is proper. Let us fix a smooth density $m$ on $Z$. Consider the following operator

$$
R: C^{\infty}\left(X_{1}\right) \rightarrow \mathcal{M}^{\infty}\left(X_{2}\right)
$$

given by

$$
R(f):=q_{2 *}\left(m \cdot q_{1}^{*} f\right)
$$

where $q_{1}^{*}$ is the usual pull-back of functions, and $q_{2 *}$ is the usual push-forward on densities. Since $q_{2}$ is a submersion the image of $R$ lies in smooth densities, and $R$ is a continuous linear operator.

All classically known Radon transforms on functions have such a form, e.g. the classical Radon transform over hyperplanes in $\mathbb{R}^{n}$ or in projective space $\mathbb{R} \mathbb{P}^{n}$, and the Radon transform for a pair of Grassmann manifolds [21]. In classical situations the manifold $Z$ is often certain incidence variety acted by a Lie group in a transitive way, and $m$ is a Haar measure.

In the above high generality we know only one non-trivial result [22]. Let us state a consequence of it. Let us assume that all the manifolds $X_{1}, X_{2}, Z$ are compact. Assume moreover that the projection

$$
T_{Z}^{*}\left(X_{1} \times X_{2}\right) \backslash \underline{0} \rightarrow T^{*} X_{2} \backslash \underline{0}
$$

is an injective immersion. Assume finally that the density $m$ on $Z$ is strictly positive everywhere. Then the Radon transform $R$ has a finite dimensional kernel. 
Now let us show that the general Radon transform on functions (4.2.1) can be considered as a special case of the general Radon transform on valuations from Section 4.1 .

First let us recall that the space of smooth densities $\mathcal{M}^{\infty}(Z)$ is a subspace of smooth valuations $V^{\infty}(Z)$. Thus we may consider the density $m$ as a valuation. Using Theorem 4.1.1 consider the operator

$$
R^{\prime}: V^{\infty}\left(X_{1}\right) \rightarrow V^{-\infty}\left(X_{2}\right)
$$

given by

$$
R^{\prime}(\phi):=q_{2 *}\left(m \cdot q_{1}^{*} \phi\right)
$$

where now $q_{1}^{*}$ and $q_{2 *}$ denote the pull-back and push-forward on valuations.

Recall that $V^{\infty}\left(X_{1}\right)$ has a closed subspace $W_{1}\left(V^{\infty}\left(X_{1}\right)\right)$ such that the quotient space $V^{\infty}\left(X_{1}\right) / W_{1}\left(V^{\infty}\left(X_{1}\right)\right)$ is canonically isomorphic to $C^{\infty}\left(X_{1}\right)$. Moreover by Proposition 3.3.5

$$
q_{1}^{*}\left(W_{1}\left(V^{\infty}\left(X_{1}\right)\right)\right) \subset W_{1}\left(V^{-\infty}(Z)\right) .
$$

Next by [7], equality $(7.3 .1), \mathcal{M}^{\infty}(Z) \cdot W_{1}\left(V^{-\infty}(Z)\right)=0$. Hence the operator $R^{\prime}$ admits a unique factorization

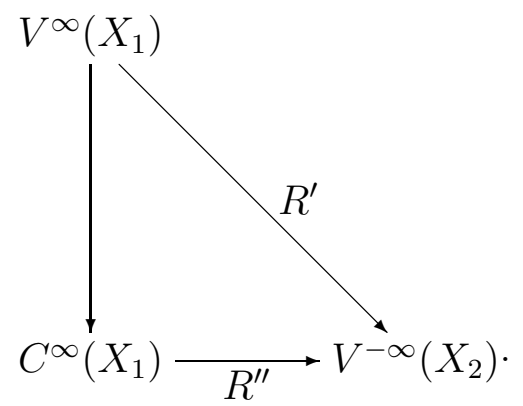

It is easy to see that the operator $R^{\prime \prime}$ takes values in smooth measures $\mathcal{M}^{\infty}(Y) \subset V^{-\infty}(Y)$ and coincides with the classical Radon transform $R$ on functions (4.2.1).

\subsection{Relations to the integral geometry of constructible functions.}

Let us discuss first the integral geometry of constructible functions in the general setting. The notion of constructible function may have slightly different meanings in various contexts; but the general ideas are the same, though the technical details may be different. Here we will use the space $\mathcal{F}(X)$ of constructible functions on a real analytic manifold $X$ described in Section 0.2,

Let $f: X \rightarrow Y$ be a real analytic map of real analytic manifolds. We have the obvious pull-back map

$$
f^{*}: \mathcal{F}(Y) \rightarrow \mathcal{F}(X)
$$

preserving the class of constructible functions. If the map $f$ is proper then we have the push-forward map

$$
f_{*}: \mathcal{F}(X) \rightarrow \mathcal{F}(Y)
$$


which is uniquely characterized by the following property: for a subanalytic subset $P \subset X$ one has

$$
f_{*}\left(\mathbb{1}_{P}\right)(y)=\chi\left(P \cap f^{-1}(y)\right)
$$

for any $y \in Y$. One can show that $f_{*}$ maps constructible functions to constructible ones (see [27], §9.7). This push-forward map $f_{*}$ on constructible functions is also called integration with respect the Euler characteristic along the fibers of $f$. In particular if $Y$ is a single point $f_{*} \phi$ is called the integral of $\phi$ with respect to the Euler characteristic and is denoted by $\int_{X} \phi$.

Let us return now to integral geometry. Let $X_{1} \stackrel{q_{1}}{\leftarrow} Z \stackrel{q_{2}}{\longrightarrow} X_{2}$ be a double fibration when $X_{1}, X_{2}, Z$ are real analytic manifolds, and $q_{1}, q_{2}$ are real analytic maps. Assume that $q_{2}$ is proper. Then we have the operator on constructible functions $R: \mathcal{F}\left(X_{1}\right) \rightarrow \mathcal{F}\left(X_{2}\right)$ given by

$$
R(\phi):=q_{2 *} q_{1}^{*} \phi
$$

which is another version of the Radon transform.

As far as we know this type of transforms (e.g. the inversion problem) in a somewhat different context of complex algebraic constructible functions on the complex projective space $\mathbb{C P}^{n}$ was studied by Viro [39]. For real projective space $\mathbb{R P}^{n}$ and for functions constructible in a more restrictive sense the problem was studies by Khovanskii and Pukhlikov [28]. Let us discuss their inversion formula in a slightly greater generality of constructible functions in the sense of Section 0.2 as it was obtained in [37]. Let $\mathbb{R} \mathbb{P}^{n}$ denote the real projective space, i.e. the space of real lines in $\mathbb{R}^{n+1}$ passing through zero. Let $\mathbb{R} \mathbb{P}^{n \vee}$ be the dual projective space, i.e. the space of linear hyperplanes in $\mathbb{R}^{n+1}$. Let $Z$ be the incidence variety, i.e.

$$
Z:=\left\{(l, H) \in \mathbb{R} \mathbb{P}^{n} \times \mathbb{R} \mathbb{P}^{n \vee} \mid l \subset H\right\} .
$$

We have the natural double fibration

$$
\mathbb{R P}^{n} \stackrel{q_{1}}{\leftarrow} Z \stackrel{q_{2}}{\longrightarrow} \mathbb{R P}^{n \vee} .
$$

The following inversion formula was proved by Khovanskii-Pukhlikov 28 for more restrictive class of constructible functions; in the present form it was proved by Schapira [37].

4.3.1 Theorem. Let $R:=q_{2 *} q_{1}^{*}: \mathcal{F}\left(\mathbb{R} \mathbb{P}^{n}\right) \rightarrow \mathcal{F}\left(\mathbb{R}^{n \vee}\right)$ be the Radon transform. Consider the (dual) Radon transform

$$
R^{t}:=q_{1 *} q_{2}^{*}: \mathcal{F}\left(\mathbb{R} \mathbb{P}^{n \vee}\right) \rightarrow \mathcal{F}\left(\mathbb{R} \mathbb{P}^{n}\right) .
$$

Then for any constructible function $\phi \in \mathcal{F}\left(\mathbb{R}^{n}\right)$ one has

$$
(-1)^{n-1} R^{t} R(\phi)=\phi+\frac{1}{2}\left((-1)^{n-1}-1\right) \cdot \int_{\mathbb{R}^{n}} \phi .
$$

4.3.2 Remark. In particular if $n$ is odd then the Radon transform $R$ is injective, and (4.3.2) is the inversion formula. If $n$ is even then the kernel of $R$ consists precisely of the constant functions. 
Now we will discuss a valuation theoretic generalization of Theorem 4.3 .1 which is the main result of this section. By Corollary 4.1.7(1) and Example 4.1.8 we have the continuous linear operator $\tilde{R}$ on generalized valuations

$$
\tilde{R}:=q_{2 *} q_{1}^{*}: V^{-\infty}\left(\mathbb{R P}^{n}\right) \rightarrow V^{-\infty}\left(\mathbb{R} \mathbb{P}^{n \vee}\right) .
$$

(Here we just take the kernel $\gamma$ to be equal to the Euler characteristic $\chi$.) Note that by Corollary 4.1.7 (2) $\tilde{R}$ maps smooth valuations to smooth ones: $\tilde{R}\left(V^{\infty}\left(\mathbb{R} \mathbb{P}^{n}\right)\right) \subset V^{\infty}\left(\mathbb{R} \mathbb{P}^{n \vee}\right)$. Similarly we define

$$
\tilde{R}^{t}:=q_{1 *} q_{2}^{*}: V^{-\infty}\left(\mathbb{R} \mathbb{P}^{n \vee}\right) \rightarrow V^{-\infty}\left(\mathbb{R P}^{n}\right)
$$

which is also continuous and maps smooth valuations to smooth ones. We have

4.3.3 Theorem. For any generalized valuations $\phi \in V^{-\infty}\left(\mathbb{R}^{n}\right)$ one has

$$
(-1)^{n-1} \tilde{R}^{t} \tilde{R}(\phi)=\phi+\frac{1}{2}\left((-1)^{n-1}-1\right)\left(\int_{\mathbb{R}^{n}} \phi\right) \cdot \chi .
$$

In particular if $n$ is odd then the Radon transform $\tilde{R}$ is injective, and (4.3.3) is the inversion formula. If $n$ is even then the kernel of $\tilde{R}$ consists precisely of the multiples of the Euler characteristic $\chi$.

In order to prove Theorem 4.3 .3 we will need some preparations. Below we will denote for brevity $\mathbb{R} \mathbb{P}^{n}$ by $\mathbb{P}^{n}$, and $\mathbb{R} \mathbb{P}^{n \vee}$ by $\mathbb{P}^{n \vee}$. Let

$$
r: \mathbb{R}^{n+1} \backslash 0 \rightarrow \mathbb{P}^{n}
$$

be the natural map.

4.3.4 Definition. A subset $K \subset \mathbb{P}^{n}$ is called convex if

(i) there exists a convex cone $\tilde{K} \subset \mathbb{R}^{n+1}$ such that $r(\tilde{K} \backslash 0)=K$;

(ii) there exists a hyperplane $H \subset \mathbb{P}^{n}$ such that $H \cap K=\emptyset$.

4.3.5 Remark. Equivalently, a subset $K \subset \mathbb{P}^{n}$ is convex if and only if there exists a hyperplane $H \subset \mathbb{P}^{n}$ which does not intersect $K$, and $K$ is a convex subset in the usual (affine) sense of affine space $\mathbb{P}^{n} \backslash H \simeq \mathbb{R}^{n}$. This condition does not depend on a choice of $H$ due to equivalence to Definition 4.3.4.

4.3.6 Definition. A closed convex subset $K \subset \mathbb{P}^{n}$ with non-empty interior is called strictly convex if

(i) $\partial K$ is smooth;

(ii) $\partial K$ has a strictly positive Gauss curvature when $K$ is considered as a subset of an affine space $\mathbb{P}^{n} \backslash H$, where $H$ is a hyperplane not intersecting $K$.

It is easy to see that the last definition is independent of a choice of hyperplane $H$. 
The dual projective space $\mathbb{P}^{n \vee}$ can naturally be identified with the projectivization of the the dual space $\left(\mathbb{R}^{n+1}\right)^{*}$. It can be identified with the set of hyperplanes in $\mathbb{P}^{n}$. Denote by

$$
r^{\vee}:\left(\mathbb{R}^{n+1}\right)^{*} \backslash\{0\} \rightarrow \mathbb{P}^{n \vee}
$$

the natural map.

For a convex compact subset $K \subset \mathbb{P}^{n}$ let us define the dual set $K^{\vee} \subset \mathbb{P}^{n \vee}$ as follows. Let $\tilde{K} \subset \mathbb{R}^{n+1}$ be a closed convex cone satisfying $r(\tilde{K} \backslash 0)=K$. $\tilde{K}$ is defined uniquely up to multiplication by -1 . Consider the dual cone in $\left(\mathbb{R}^{n+1}\right)^{*}$

$$
\tilde{K}^{o}:=\left\{\xi \in\left(\mathbb{R}^{n+1}\right)^{*} \mid \xi(x) \geq 0 \forall x \in \tilde{K}\right\} .
$$

Define

$$
K^{\vee}:=r^{\vee}\left(\tilde{K}^{o}\right) .
$$

If $K$ has non-empty interior then $K^{\vee}$ is also convex in the sense of Definition 4.3.4. In this case $\left(K^{\vee}\right)^{\vee}=K$. It is easy to see that the dual of a strictly convex set is strictly convex.

The following another description of $K^{\vee}$ will be useful. Assume that $K$ has non-empty interior. Then $K^{\vee}$ is equal to the set of hyperplanes which do not intersect the interior of $K$.

Before the proof of Theorem 4.3 .3 we will prove the following technical proposition.

4.3.7 Proposition. Let $K \subset \mathbb{P}^{n}$ be a closed convex set. Then

$$
R\left(\Xi_{\mathcal{P}}\left(\mathbb{1}_{K}\right)\right)=\Xi_{\mathcal{P}}\left(\mathbb{1}_{\mathbb{P}^{n \vee} \backslash \operatorname{int}\left(K^{\vee}\right)}\right),
$$

where $\operatorname{int}\left(K^{\vee}\right)$ denotes the interior of $K^{\vee}$.

4.3.8 Remark. By the abuse of notation we denote by $\Xi_{\mathcal{P}}$ the map defined not only on the space $\mathcal{F}(X)$ of constructible functions (in the subanalytic sense), but also on indicator functions of convex compact sets and also of compact submanifolds with corners (for all of them the notion of normal cycle is well defined too). The non-trivial results from the previous sections will be applied for indicator functions of convex sets with smooth strictly convex boundary. These modifications of the map $\Xi_{\mathcal{P}}$ are purely formal, and there is no need to introduce separate notation for them.

Proof of Proposition 4.3.7. Step 1. Let us show that it is enough to prove the statement for $\partial K$ being smooth strictly convex.

For a closed convex set $K$ there exists a sequence $\left\{K_{j}\right\}$ of strictly convex sets which converges to $K$ in the Hausdorff metric. Then it is easy to see that $K_{j}^{\vee} \rightarrow K^{\vee}$ in the Hausdorff metric. But then

$$
\begin{array}{r}
\Xi_{\mathcal{P}}\left(\mathbb{1}_{K_{j}}\right) \rightarrow \Xi_{\mathcal{P}}\left(\mathbb{1}_{K}\right) \text { in } V^{-\infty}\left(\mathbb{P}^{n}\right), \\
\Xi_{\mathcal{P}}\left(\mathbb{1}_{K_{j}^{\vee}}\right) \rightarrow \Xi_{\mathcal{P}}\left(\mathbb{1}_{K^{\vee}}\right) \text { in } V^{-\infty}\left(\mathbb{P}^{n \vee}\right) .
\end{array}
$$

But for a compact convex set $B \subset \mathbb{P}^{n \vee}$ with non-empty interior, $B \in \mathcal{P}\left(\mathbb{P}^{n \vee}\right)$, one readily has

$$
\Xi_{\mathcal{P}}\left(\mathbb{1}_{\mathbb{P} n \vee} \backslash \operatorname{int}(B)\right)=\chi-\sigma\left(\Xi_{\mathcal{P}}\left(\mathbb{1}_{B}\right)\right)
$$


where the operator $\sigma: V^{-\infty}\left(\mathbb{P}^{n \vee}\right) \rightarrow V^{-\infty}\left(\mathbb{P}^{n \vee}\right)$ is a continuous linear operator introduced in [7] called the Euler-Verdier involution. Hence

$$
\Xi_{\mathcal{P}}\left(\mathbb{1}_{\mathbb{P} n \vee \backslash i n t\left(K_{j}^{\vee}\right)}\right) \rightarrow \Xi_{\mathcal{P}}\left(\mathbb{1}_{\mathbb{P} n \vee} \backslash \operatorname{int}\left(K^{\vee}\right)\right) \text { in } V^{-\infty}\left(\mathbb{P}^{n \vee}\right)
$$

Thus since $R: V^{-\infty}\left(\mathbb{P}^{n}\right) \rightarrow V^{-\infty}\left(\mathbb{P}^{n \vee}\right)$ is continuous in the weak topology we may assume that $\partial K$ is smooth and strictly convex.

Step 2. $R\left(\Xi_{\mathcal{P}}\left(\mathbb{1}_{K}\right)\right)$ vanishes on $\operatorname{int}\left(K^{\vee}\right)$. Indeed since $\operatorname{int}\left(K^{\vee}\right)$ consists of hyperplanes in

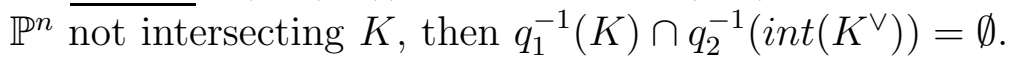

Step 3. Let us show that the restriction of $R\left(\Xi_{\mathcal{P}}\left(\mathbb{1}_{K}\right)\right)$ to the open set $\mathbb{P}^{n \vee} \backslash K^{\vee}$ is equal to $\chi$.

First let us show that

$$
q_{2}: q_{1}^{-1}(\partial K) \cap q_{2}^{-1}\left(\mathbb{P}^{n \vee} \backslash K^{\vee}\right) \rightarrow \mathbb{P}^{n \vee} \backslash K^{\vee}
$$

is a submersion. Let us fix $x_{0} \in \partial K, H_{0} \in \mathbb{P}^{n \vee} \backslash K^{\vee}$ such that $x_{0} \in H_{0}$. Let us denote by $A_{1}$ the tangent space of $\mathbb{P}^{n}$ at $x_{0}, A_{2}$ the tangent space of $\mathbb{P}^{n \vee}$ at $H_{0}, B$ the tangent space of $\partial K$ at $x_{0}, C$ the tangent space of $Z$ at $\left(x_{0}, H_{0}\right)$, and $D$ the tangent space of $q_{1}^{-1}(\partial K)$ at $\left(x_{0}, H_{0}\right)$. Then we have

$$
\begin{array}{r}
B \subset A_{1} \stackrel{d q_{1}}{\leftarrow} C \stackrel{d q_{2}}{\longrightarrow} A_{2}, \\
D \subset C .
\end{array}
$$

We want to show

$$
d q_{2}(D)=A_{2}
$$

But clearly $D=\left(d q_{1}\right)^{-1}(B)$. Denote also $F:=\operatorname{Ker}\left(d q_{2}\right)$. Then (4.3.5) is equivalent to

$$
\left(d q_{1}^{-1}\right)(B)+F=C .
$$

This is equivalent to

$$
B+\left(d q_{1}\right)(F)=A_{1}
$$

But $F$ is the tangent space at $H_{0}$ of the fiber $q_{2}^{-1}\left(H_{0}\right)$. Hence $\left(d q_{1}\right)(F)$ is the tangent space of $q_{1}\left(q_{2}^{-1} H_{0}\right)$ at $x_{0}$. But obviously

$$
q_{1}\left(q_{2}^{-1} H_{0}\right)=\left\{x \in \mathbb{P}^{n} \mid x \in H_{0}\right\}=H_{0} .
$$

The condition (4.3.6) means that the hyperplane $H_{0}$ is transversal to $\partial K$ at $x_{0}$. But it is easy to see that any hyperplane $H_{0} \in \mathbb{P}^{n \vee} \backslash K^{\vee}$ is transversal to $\partial K$ since $K$ is convex. Thus we have shown that the map (4.3.4) is a submersion. 
This implies that for any point $H \in \mathbb{P}^{n \vee} \backslash K^{\vee}$ there exists a neighborhood $\mathcal{U}$ of $H$, a neighborhood $\mathcal{V}$ of $q_{1}^{-1}(K) \cap q_{2}^{-1}(\mathcal{U})$ such that $\mathcal{V} \subset q_{2}^{-1}(\mathcal{U})$, and a diffeomorphism

$$
\phi: \mathcal{V} \sim \mathcal{U} \times \mathbb{R}^{n-1}
$$

such that $\phi\left(q_{1}^{-1}(K) \cap q_{2}^{-1}(\mathcal{U})\right)=\mathcal{U} \times B$ where $B \subset \mathbb{R}^{n-1}$ is the closed unit ball, and such that the following diagram is commutative:

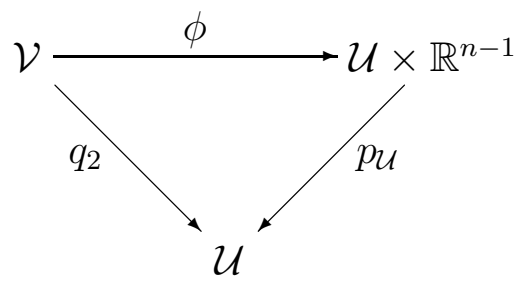

where $p_{\mathcal{U}}: \mathcal{U} \times \mathbb{R}^{n-1} \rightarrow \mathcal{U}$ is the natural projection. Then Proposition 3.3.4 and Lemma 3.6.4 imply that the restriction of $q_{2 *} q_{1}^{*}\left(\Xi_{\mathcal{P}}\left(\mathbb{1}_{K}\right)\right)=q_{2 *}\left(\Xi_{\mathcal{P}}\left(\mathbb{1}_{q_{1}^{-1}(K)}\right)\right)$ to $\mathcal{U}$ is equal to $p_{\mathcal{U} *}\left(\Xi_{\mathcal{P}}\left(\mathbb{1}_{\mathcal{U} \times B}\right)\right)=$ $\chi(B) \cdot \chi=\chi$. Hence the restriction of $R\left(\Xi_{\mathcal{P}}\left(\mathbb{1}_{K}\right)\right)$ to $\mathbb{P}^{n \vee} \backslash K^{\vee}$ is equal to $\chi$.

Step 4. Now let us fix a point $H^{0} \in \partial K^{\vee}$, and show that the restriction of $R\left(\Xi_{\mathcal{P}}\left(\mathbb{1}_{K}\right)\right)$ to a small neighborhood of $H^{0}$ is equal to $\Xi_{\mathcal{P}}\left(\mathbb{1}_{\mathbb{P}^{n \vee} \backslash \operatorname{int}\left(K^{\vee}\right)}\right)$.

Let $\tilde{K} \subset \mathbb{R}^{n+1}$ be a closed convex cone over $K$. We can choose a coordinate system $\left\{\left(x_{1}, \ldots, x_{n+1}\right)\right\}$ in $\mathbb{R}^{n+1}$ such that the hyperplane $\left\{\left(x_{1}, \ldots, x_{n}, 0\right)\right\}$ intersects $\tilde{K}$ only at 0 , and such that the point $(0, \ldots, 0,1) \in \operatorname{int}(\tilde{K})$.

Let $\left\{y_{1}, \ldots, y_{n+1}\right\}$ be the dual coordinates in $\left(\mathbb{R}^{n+1}\right)^{*}$. The the dual cone $\tilde{K}^{o}$ is given by

$$
\tilde{K}^{o}=\left\{\left(y_{1}, \ldots, y_{n+1}\right) \mid \sum_{i=1}^{n+1} x_{i} y_{i} \geq 0 \text { for all }\left(x_{1}, \ldots, x_{n+1}\right) \in \tilde{K}\right\} .
$$

It is clear that if $\left(y_{1}, \ldots, y_{n+1}\right) \in \tilde{K}^{o} \backslash\{0\}$ then $y_{n+1}>0$. In particular the hyperplane $\left\{\left(y_{1}, \ldots, y_{n}, 0\right)\right\}$ intersects $\tilde{K}^{o}$ only at 0 .

Thus we have shown that $K$ is contained in the affine chart of $\mathbb{P}^{n}$ given by $\left\{\left[x_{1}: \cdots: x_{n}\right.\right.$ : $1]\}$ in homogeneous coordinates, and $K^{\vee}$ is contained in $\left\{\left[y_{1}: \cdots: y_{n}: 1\right]\right\}$. We will identify both these charts with $\mathbb{R}^{n}$ using the coordinates $\left(x_{1}, \ldots, x_{n}\right)$ and $\left(y_{1}, \ldots, y_{n}\right)$ respectively. In these coordinates

$$
\begin{array}{r}
(0, \ldots, 0) \in K, \\
K^{\vee}=\left\{\left(y_{1}, \ldots, y_{n}\right) \mid \sum_{i+1}^{n} x_{i} y_{i} \geq-1 \text { for all }\left(x_{1}, \ldots, x_{n}\right) \in K\right\} .
\end{array}
$$

Notice that $-K^{\vee}=\left\{\left(y_{1}, \ldots, y_{n}\right) \mid \sum_{i+1}^{n} x_{i} y_{i} \leq 1\right.$ for all $\left.\left(x_{1}, \ldots, x_{n}\right) \in K\right\}$ is the dual body of $K$ in the usual linear sense.

We will reduce our statement to Proposition 3.6.5 applying certain appropriate diffeomorphism. More precisely we will show that there exist a neighborhood $\mathcal{U} \subset \mathbb{P}^{n \vee}$ of an arbitrary fixed point $H^{0} \in \partial K^{\vee}$, a neighborhood $\mathcal{V}$ of $q_{1}^{-1}(K) \cap q_{2}^{-1}(\mathcal{U})$ such that $\mathcal{V} \subset q_{2}^{-1}(\mathcal{U})$, and open imbeddings

$$
\begin{array}{r}
\phi: \mathcal{V} \rightarrow \mathbb{R}^{n} \times \mathbb{R}^{n-1}, \\
\psi: \mathcal{U} \rightarrow \mathbb{R}^{n}
\end{array}
$$


such that the following two conditions are satisfied:

(1) the diagram is commutative:

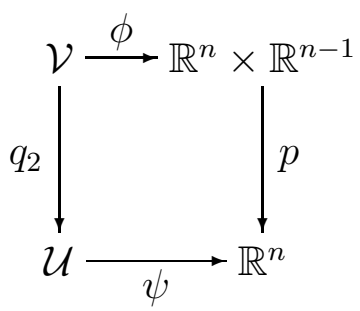

where $p: \mathbb{R}^{n} \times \mathbb{R}^{n-1} \rightarrow \mathbb{R}^{n}$ is the projection onto the first factor;

(2) $\phi\left(q_{1}^{-1}(K) \cap q_{2}^{-1}(\mathcal{U})\right)=: A$ is a closed convex subset of $\mathbb{R}^{n} \times \mathbb{R}^{n-1}$ with smooth strictly convex boundary, and the restriction $\left.p\right|_{A}$ is proper.

Clearly if we will show the above, Proposition 3.6.5 finishes the proof of our proposition.

For $\varepsilon>0$ let us consider the map

$$
\phi_{1}: \partial K^{\vee} \times(-\varepsilon, \varepsilon) \rightarrow \mathbb{R}^{n}
$$

given by $\phi_{1}(s, t):=(1+t)^{-1} s$. Clearly $\phi_{1}$ is a diffeomorphism onto an open neighborhood of $\partial K^{\vee}$ in $\mathbb{R}^{n}$ for $\varepsilon$ small enough.

For any point $s \in \partial K^{\vee}$ there exists a unique point $x \in \partial K$ such that $\langle s, x\rangle=-1$. This defines a map

$$
\Phi: \partial K^{\vee} \rightarrow \partial K
$$

which is a diffeomorphism since $\partial K$ is strictly convex. For $y \in \mathbb{R}^{n} \backslash\{0\}$ let us denote the hyperplane

$$
H_{y}:=\left\{\left(x_{1}, \ldots, x_{n}\right) \in \mathbb{R}^{n} \mid \sum_{i=1}^{n} x_{i} y_{i}=-1\right\} .
$$

If $H=\phi_{1}(s, t), s \in \partial K^{\vee}, t \in(-\varepsilon, \varepsilon)$, then $x \in H_{s}$ if and only if

$$
<s, x>=-(1+t)
$$

For $s \in \partial K^{\vee}$ belonging to a small neighborhood of the fixed point $H^{0} \in \partial K^{\vee}$ let us choose a basis $\xi_{1}(s), \ldots, \xi_{n-1}(s)$ of $s^{\perp}$ which depends smoothly on $s$. Then any $x \in H_{\phi_{1}(s, t)}$ has a decomposition

$$
x=\sum_{j=1}^{n-1} \lambda_{j} \xi_{j}(s)+(1+t) \Phi(s)
$$

with $\lambda_{j} \in \mathbb{R}$ (the coefficient of $\Phi(s)$ is equal to $1+t$ due to (4.3.8)). Hence on the incidence variety $Z$ near the pair $\left(\Phi\left(H^{0}\right), H^{0}\right)$ we have a coordinate system given by $\left(s ; \lambda_{1}, \ldots, \lambda_{n-1} ; t\right)$.

Let us describe the set $q_{1}^{-1}(K)$ in these coordinates. For any $s \in \partial K^{\vee}$ there exists a unique function

$$
F_{s}: s^{\perp} \rightarrow \mathbb{R}
$$


such that near $\Phi(s)$ we have

$$
K=\left\{h-\tau \Phi(s) \mid h \in s^{\perp}, \tau \geq F_{s}(h)\right\} .
$$

This function $F_{s}$ is $C^{\infty}$-smooth and strictly convex. Moreover the function

$$
F(s, h):=F_{s}(h)
$$

is $C^{\infty}$-smooth on the set $\left\{(s, h) \mid s \in \partial K^{\vee}, h \in s^{\perp}\right\}$ near $s=H^{0}, h=0$. Also $0 \in s^{\perp}$ is a critical point of $F_{s}$, and $F_{s}(0)=-1$.

Assume we are given a pair $(x, y) \in Z \subset \mathbb{P}^{n} \times \mathbb{P}^{n \vee}$ corresponding to $\left(s ; \lambda_{1}, \ldots, \lambda_{n-1} ; t\right)$. Then $x \in K$ if and only if

$$
-t \geq 1+F_{s}\left(\sum_{j} \lambda_{j} \xi_{j}(s)\right)
$$

Indeed by (4.3.9) $x=\sum \lambda_{j} \xi_{j}(s)+(1+t) \Phi(s)$. Then by (4.3.10), $x \in K$ if and only if $-(1+t) \geq F_{s}\left(\sum \lambda_{j} \xi_{j}(s)\right)$.

Now let us denote for brevity

$$
G_{s}\left(\lambda_{1}, \ldots, \lambda_{n-1}\right):=1+F_{s}\left(\sum_{j=1}^{n-1} \lambda_{j} \xi_{j}(s)\right) .
$$

Thus we have shown that in the above coordinates on $Z$ near $\left(0, H^{0}\right)$ the set $q_{1}^{-1}(K)$ is given by

$$
q_{1}^{-1}(K)=\left\{\left(s ; \lambda_{1}, \ldots, \lambda_{n-1} ; t\right) \in \partial K^{\vee} \times \mathbb{R}^{n-1} \times \mathbb{R} \mid-t \geq G_{s}\left(\lambda_{1}, \ldots, \lambda_{n-1}\right)\right\} .
$$

It is clear that the map $\left(s, \lambda_{1}, \ldots, \lambda_{n-1}\right) \mapsto G_{s}\left(\lambda_{1}, \ldots, \lambda_{n-1}\right)$ is $C^{\infty}$-smooth, and when $s$ is fixed the function $G_{s}$ is strictly convex. In particular for fixed $s, G_{s}$ is a Morse function. Notice also that $G_{s}$ has a minimum at $0 \in \mathbb{R}^{n-1}$, and $G_{s}(0)=0$.

By the Morse lemma with parameters (see e.g. [16], p. 97) there exists a $C^{\infty}$ map

$$
D: \partial K^{\vee} \times \mathbb{R}^{n-1} \rightarrow \partial K^{\vee} \times \mathbb{R}^{n-1}
$$

of the form $D\left(s ; \mu_{1}, \ldots, \mu_{n-1}\right)=\left(s ; \lambda_{1}, \ldots, \lambda_{n-1}\right)$ such that for $s$ close to $H^{0}$ the map $\mathbb{R}^{n-1} \rightarrow$ $\mathbb{R}^{n-1}$ given by $\left(\mu_{1}, \ldots, \mu_{n-1}\right) \mapsto D\left(s ; \mu_{1}, \ldots, \mu_{n-1}\right)=: D_{s}\left(\mu_{1}, \ldots, \mu_{n-1}\right)$ is a diffeomorphism of a neighborhood of $0 \in \mathbb{R}^{n-1}$, and

$$
\left(G_{s} \circ D_{s}\right)\left(\mu_{1}, \ldots, \mu_{n-1}\right)=\mu_{1}^{2}+\cdots+\mu_{n-1}^{2} .
$$

In the new coordinates defined by

$$
\left(s ; \mu_{1}, \ldots, \mu_{n-1} ; \tau\right):=\left(s ; D_{s}^{-1}\left(\lambda_{1}, \ldots, \lambda_{n-1}\right) ;-t\right)
$$

the set $q_{1}^{-1}(K)$ is locally given by

$$
q_{1}^{-1}(K)=\left\{\left(s ; \mu_{1}, \ldots, \mu_{n-1} ; \tau\right) \mid s \in \partial K^{\vee}, \tau \geq \mu_{1}^{2}+\cdots+\mu_{n-1}^{2}\right\} .
$$


Moreover in these coordinates the map $q_{2}: Z \rightarrow \mathbb{P}^{n \vee}$ is given by

$$
q_{2}\left(s ; \mu_{1}, \ldots, \mu_{n-1} ; \tau\right)=\phi_{1}(s,-\tau) .
$$

Let us fix a coordinate system $\left\{\left(s_{1}, \ldots, s_{n-1}\right)\right\}$ on $\partial K^{\vee}$ near $H^{0}$ such that $(0, \ldots, 0)$ corresponds to $H^{0}$. Finally let us introduce the last our coordinate system $\left\{\left(s_{1}, \ldots, s_{n-1} ; \mu_{1}, \ldots, \mu_{n-1} ; r\right)\right\}$ on $Z$ near $\left(\Phi\left(H^{0}\right), H^{0}\right)$ when $r$ is defined by

$$
r:=\tau+\sum_{j=1}^{n-1} s_{j}^{2}
$$

Then $\left\{\left(s_{1}, \ldots, s_{n-1} ; r\right)\right\}$ is a coordinate system on $\mathbb{P}^{n \vee}$ near $H^{0}$. Then $q_{1}^{-1}(K)$ is given by

$$
q_{1}^{-1}(K)=\left\{\left(s_{1}, \ldots, s_{n-1} ; \mu_{1}, \ldots, \mu_{n-1} ; r\right) \mid r \geq \sum_{j=1}^{n-1} s_{j}^{2}+\sum_{j=1}^{n-1} \mu_{j}^{2}\right\}
$$

and the map $q_{2}$ is given by

$$
q_{2}\left(s_{1}, \ldots, s_{n-1} ; \mu_{1}, \ldots, \mu_{n-1} ; r\right)=\left(s_{1}, \ldots, s_{n-1} ; r\right) .
$$

Thus the formula (4.3.11) defines a Euclidean ball in $\mathbb{R}^{2 n-1}$, and the map $q_{2}$ given by (4.3.12) is just a projection. This coordinate system gives the maps $\phi, \psi$ as promised in the beginning of Step 4. Hence Step 4 is completed.

Steps 1-4 imply Proposition 4.3.7. Q.E.D.

Proof of Theorem 4.3.3. The linear operator

$$
\tilde{R}^{t} \tilde{R}: V^{-\infty}\left(\mathbb{P}^{n}\right) \rightarrow V^{-\infty}\left(\mathbb{P}^{n}\right)
$$

is continuous in the weak topology by Corollary 4.1.7 and Example 4.1.8(2). It is not hard to see (as in [7], Section 8.1) that linear combinations of valuations of the form $\Xi_{\mathcal{P}}\left(\mathbb{1}_{K}\right)$, with $K \subset \mathbb{P}^{n}$ being a compact convex polyhedral subset, are dense in $V^{-\infty}\left(\mathbb{P}^{n}\right)$. Hence it is sufficient to prove the inversion formula (4.3.3) for such valuations. By Proposition 4.3.7 the operators $\tilde{R}, \tilde{R}^{t}$ coincide on such valuations with the Radon transform on constructible functions with respect to the Euler characteristic. Note also that again by Proposition 4.3.7 the operator $\tilde{R}$ maps valuations of this form on $\mathbb{P}^{n}$ to valuations of this form on $\mathbb{P}^{n \vee}$ : indeed if $K \subset \mathbb{P}^{n}$ is a compact convex polyhedral subset then the dual $K^{\vee}$ is also compact convex and polyhedral. But by the Khovanskii-Pukhlikov theorem [28] the formula (4.3.3) is satisfied for the integration of constructible functions with respect to the Euler characteristic. Q.E.D.

\subsection{Relations to the Crofton style integral geometry.}

In this section we explain the relation of operations on valuations to yet another classical kind of integral geometry. However the exposition in this section is less formal and rigorous than in the rest of the article. 
Let us remind the classical Crofton intersection formula and the general kinematic formulas of Chern [18]. (For the Crofton formula we refer to Schneider's book [38], §4.5; for the kinematic formulas see also Santalo's book [35] and references therein.)

First we will need to remind the notion of intrinsic volume. Consider the standard Euclidean space $\mathbb{R}^{n}$. Let $M \subset \mathbb{R}^{n}$ be a compact submanifold with corners. For $\varepsilon>0$ let us denote by $M_{\varepsilon}$ the $\varepsilon$-neighborhood of $M$ (or $\varepsilon$-tube in some terminology). For sufficiently small $\varepsilon>0$ the volume of $M_{\varepsilon}$ is a polynomial of degree $n$ with respect to $\varepsilon$ :

$$
\operatorname{vol}\left(M_{\varepsilon}\right)=\sum_{i=0}^{n} \varepsilon^{n-i} \kappa_{n-i} V_{i}(M)
$$

where $\kappa_{j}$ is the $j$-dimensional volume of the $j$-dimensional unit ball. The coefficient $V_{i}(M)$ is called the $i$-th intrinsic volume of $M$. It is not hard to see that $V_{i}$ is a smooth valuation. $V_{0}$ is the Euler characteristic, and $V_{n}$ is the $n$-dimensional volume. But the most surprising property of $V_{i}$ is due to $\mathrm{H}$. Weyl [40]; it says that $V_{i}(M)$ is independent of an isometric imbedding of $M$ into $\mathbb{R}^{n}$. It fact $V_{i}$ can be written in terms of the curvature tensor of $M$ (see Weyl's paper [40], see also [26]).

Let us denote by $\overline{O(n)}$ the group of all affine isometries of $\mathbb{R}^{n}$. Let $m$ denote the Haar measure on $\overline{O(n)}$ (we do not specify the normalization of it since we will not write down here the constants anyway). Let us denote by $\mathcal{A} G r_{k, n}$ the Grassmannian of affine $k$-dimensional subspaces in $\mathbb{R}^{n}$. The group $\overline{O(n)}$ acts transitively on it. Let $\mu$ denote a Haar measure of it with respect to the action of the group $\overline{O(n)}$. Then the Crofton intersection formula says that

$$
\int_{\mathcal{A} G r_{k, n}} V_{i}(M \cap E) d \mu(E)=\alpha_{i, k, n} V_{n+i-k}
$$

where $\alpha_{i, k, n}$ depends on $i, k, n$ only.

The general kinematic formula of Chern [18] says that for two compact submanifolds with boundary $M, N$ one has

$$
\int_{\overline{O(n)}} V_{i}(M \cap g(N)) d m(g)=\sum_{j} c_{i, j, n} V_{j}(M) V_{n+i-j}(N)
$$

where the constants $c_{i, j, n}$ depend only on $i, j, n$ and can be written down explicitly.

Let us explain how to rewrite the left hand side in the formulas of Crofton and Chern using the operations of pull-back, push-forward, and the product of valuations.

Let us start with the Crofton formula. Let us denote by

$$
Z:=\left\{(x, E) \in \mathbb{R}^{n} \times \mathcal{A} G r_{k, n} \mid x \in E\right\}
$$

the incidence variety. We have the double fibration $\mathbb{R}^{n} \stackrel{q_{1}}{\leftarrow} Z \stackrel{q_{2}}{\longrightarrow} \mathcal{A} G r_{k, n}$. Clearly $q_{1}$ is proper. 
One can see the following formula for the left hand side of (4.4.1)

$$
\int_{\mathcal{A} G r_{k, n}} V_{i}(M \cap E) d \mu(E)=\left(V_{i} \cdot q_{1 *} q_{2}^{*} \mu\right)(M)
$$

where the pull-back $q_{2}^{*}$, the push-forward $q_{1 *}$, and the product are taken in the space of valuations. Let us also notice that the formula (4.4.3) holds in a greater generality: not only for a Haar measure $\mu$ but for any smooth measure on the affine Grassmannian.

Let us return to the general kinematic formula (4.4.2). The left hand side of it can be rewritten as follows. Let us consider the double fibration

$$
\mathbb{R}^{n} \stackrel{p}{\leftarrow} \overline{O(n)} \times \mathbb{R}^{n} \stackrel{a}{\rightarrow} \mathbb{R}^{n}
$$

where $p$ is the projection $p(g, x)=x$, and $a$ is the action map $a(g, x)=g(x)$.

$$
\int_{\overline{O(n)}} V_{i}(M \cap g(N)) d m(g)=\left(p_{*}\left(a^{*}\left[\Xi_{\mathcal{P}}\left(\mathbb{1}_{N}\right)\right] \cdot\left[m \otimes V_{i}\right]\right)\right)(M)
$$

where $\triangle$ in the right hand side is the exterior product on valuations introduced in Section 2 . Observe that the operator $\left[\phi \mapsto p_{*}\left(a^{*} \phi \cdot\left(m \otimes V_{i}\right)\right)\right]$ takes values in $\overline{O(n)}$-invariant valuations.

Finally we would like to rewrite in this language some integral geometric expressions in complex integral geometry. They are not fully understood and still under investigation. The case of the affine complex space $\mathbb{C}^{n}$ is understood better (see [4], [15]), and we consider here the less studied case of the complex projective space $\mathbb{C P}^{n}$. To introduce the complex version of the Crofton formula (actually only the left hand side of it, as the right hand side is still to be understood), let us denote by ${ }^{\mathbf{C}} G r_{k+1, n+1}$ the Grassmannian of complex linear subspaces in $\mathbb{C}^{n+1}$ of complex dimension $k+1$. Every element of it can be identified with the $k$-dimensional complex projective subspace in $\mathbb{C P}^{n}$. This Grassmannian is acted by the unitary group $U(n+1)$ in a transitive way. We will denote by $\mu$ a Haar measure on the Grassmannian. Let $M \subset \mathbb{C P}^{n}$ be a smooth submanifold with boundary (or even with corners). In this notation, one would like to have a closed formula for

$$
\int_{E \in \in^{\mathbf{C}} G r_{k+1, n+1}} V_{i}(M \cap E) d \mu(E) .
$$

Let us consider the double fibration

$$
\mathbb{C P}^{n} \stackrel{q_{1}}{\leftarrow} Z \stackrel{q_{2}}{\longrightarrow}{ }^{\mathbf{C}} G r_{k+1, n+1}
$$

where $Z:=\left\{(l, E) \in \mathbb{C P}^{n} \times{ }^{\mathrm{C}} G r_{k+1, n+1} \mid l \subset E\right\}$ is the incidence variety again. Then the integral (4.4.5) can be rewritten as

$$
\left(V_{i} \cdot q_{1 *} q_{2}^{*}(\mu)\right)(M) .
$$

This expression is an $U(n+1)$-invariant valuation on $\mathbb{C P}^{n}$. The similarity with the expression (4.4.3) in the Euclidean case is evident. Notice that in the recent article [1] a closed formula for (4.4.5) in the case $i=0$ has been obtained.

Analogously the left hand sides of Crofton and kinematic type formulas can be written also on the complex hyperbolic space where they are also not well understood. 


\section{Appendix.}

In this appendix we prove that some pairs of the Grassmannians satisfy the assumptions of Corollary 4.1.7 (see Proposition 5.1.3 below).

Let $\mathbb{K}$ denote either $\mathbb{R}, \mathbb{C}$, or $\mathbb{H}$ (where $\mathbb{H}$ is the non-commutative field of quaternions). Let $V$ be a $\mathbb{K}$-vector space of $\mathbb{K}$-dimension $n$. Let ${ }^{\mathbb{K}} G r_{k}$ denote the Grassmannian of $\mathbb{K}$-linear subspaces in $V$ of $\mathbb{K}$-dimension $k$. Fix now $k, m$ with $0<k<m<n$. Let

$$
Z \subset{ }^{\mathbb{K}} G r_{k} \times{ }^{\mathbb{K}} G r_{m}
$$

be the incidence variety, i.e. $Z:=\{(l, H) \mid l \subset H\}$. It is well known that there exist canonical isomorphisms

$$
\begin{array}{r}
T_{l}\left({ }^{\mathbb{K}} G r_{k}\right)=H o m_{\mathbb{K}}(l, V / l)=l^{*} \otimes_{\mathbb{K}} V / l, \\
T_{H}\left({ }^{\mathbb{K}} G r_{m}\right)=\operatorname{Hom}_{\mathbb{K}}(H, V / H)=H^{*} \otimes_{\mathbb{K}} V / H .
\end{array}
$$

Notice that if $\mathbb{K}=\mathbb{H}$, we consider $V, l, H$ as right $\mathbb{K}$-vector spaces. Then

$$
l^{*}:=\operatorname{Hom}_{\mathbb{K}}(l, \mathbb{K}) \stackrel{\sim}{\rightarrow} \operatorname{Hom}_{\mathbb{R}}(l, \mathbb{R})
$$

is a left $\mathbb{K}$-vector space, and similarly for $H^{*}$.

For a point $(l, H) \in Z$ let us denote by

$$
\begin{aligned}
i: l & \hookrightarrow H, \\
p: V / l & \rightarrow V / H
\end{aligned}
$$

the natural maps.

5.1.1 Proposition. For $(l, H) \in Z$ the tangent space $T_{(l, H)} Z$ is naturally isomorphic to the space of pairs

$$
\left.\left.(\xi, \eta) \in T_{l}{ }^{\mathbb{K}} G r_{k}\right) \oplus T_{H}{ }^{\mathbb{K}} G r_{m}\right)=H o m_{\mathbb{K}}(l, V / l) \oplus H o m_{\mathbb{K}}(H, V / H)
$$

which make the following diagram commutative

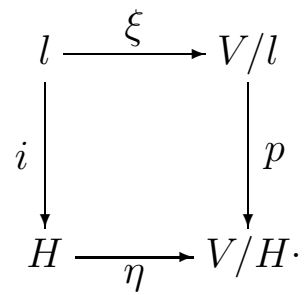

Proof. Let us fix splittings

$$
\begin{array}{r}
H=l \oplus A, \\
V=H \oplus B=l \oplus A \oplus B .
\end{array}
$$

Consider the open neighborhood of $l$ (resp. $H$ ) consisting of subspaces which are graphs of $\mathbb{K}$-linear maps

$$
l \rightarrow A \oplus B,(\text { resp. } H \rightarrow B)
$$


Given such $\phi: l \rightarrow A \oplus B, \psi: H \rightarrow B$, let us denote by $\tilde{\phi} \in{ }^{\mathbb{K}} G r_{k}, \tilde{\psi} \in{ }^{\mathbb{K}} G r_{m}$ the corresponding subspaces. The map $\phi$ has the form $\phi=\left(\phi_{A}, \phi_{B}\right)$ with

$$
\phi_{A}: l \rightarrow A, \phi_{B}: l \rightarrow B
$$

Then $\tilde{\phi} \subset \tilde{\psi}$ if and only if for any $x \in l$ the vector $\left(x, \phi_{A}(x), \phi_{B}(x)\right)$ belongs to the graph of $\psi$. Namely there exists $y=(z, a) \in H=l \oplus A$ such that

$$
\left(x, \phi_{A}(x), \phi_{B}(x)\right)=(z, a, \psi(z, a)) .
$$

Equivalently $z=x, a=\phi_{A}(x)$, and

$$
\phi_{B}(x)=\psi\left(x, \phi_{A}(x)\right)=\psi\left(x, 0_{A}\right)+\psi\left(0_{l}, \phi_{A}(x)\right) .
$$

In order to describe the tangent space $T_{(l, H)} Z$ we have to assume that $\phi, \psi$ are infinitesimally small. Then in (5.1.7) we have to neglect the summand $\psi\left(0_{l}, \phi_{A}(x)\right)$ which has the second order of smallness. Thus infinitesimally small $\phi, \psi$ satisfy

$$
\phi_{B}(x)=\psi\left(x, 0_{A}\right) \text { for any } x \in l \text {. }
$$

Equivalently

$$
p \circ \phi=\psi \circ i \text {. }
$$

This proves the proposition. Q.E.D.

By Proposition 5.1.1 we have the short exact sequence

$$
0 \rightarrow T_{(l, H)} Z \rightarrow\left(l^{*} \otimes_{\mathbb{K}} V / l\right) \oplus\left(H^{*} \otimes_{\mathbb{K}} V / H\right) \rightarrow l^{*} \otimes_{\mathbb{K}} V / H \rightarrow 0
$$

where the third arrow is $\left(i d_{l^{*}} \otimes p\right) \oplus\left(i^{*} \otimes-i d_{V / H}\right)$. Let us describe now the fiber over $(l, H)$ of the conormal bundle of $Z \subset{ }^{\mathbb{K}} G r_{k} \times{ }^{\mathbb{K}} G r_{m}$. Clearly this fiber is equal to $\left(T_{(l, H)} Z\right)^{\perp}$. Observe also that $(V / l)^{*}=l^{\perp},(V / H)^{*}=H^{\perp}$. Then taking the dual sequence of (5.1.8) we immediately deduce the following corollary.

5.1.2 Corollary. The fiber $\left(T_{(l, H)} Z\right)^{\perp}$ of the conormal bundle of $Z \subset{ }^{\mathbb{K}} G r_{k} \times{ }^{\mathbb{K}} G r_{m}$ over a point $(l, H) \in Z$ is canonically isomorphic to the image of the injective map

$$
l \otimes_{\mathbb{K}} H^{\perp} \rightarrow\left(l \otimes_{\mathbb{K}} l^{\perp}\right) \oplus\left(H \otimes_{\mathbb{K}} H^{\perp}\right)
$$

where the map is $\left(i d_{l} \otimes p^{*}\right) \oplus\left(i \otimes-i d_{H^{\perp}}\right)$.

Now we will prove the main result of this appendix.

5.1.3 Proposition. Let $k=1$ or $n-1$. Let $m \neq k$. Then the natural projection

$$
f: T_{Z}^{*}\left({ }^{\mathbb{K}} G r_{k} \times{ }^{\mathbb{K}} G r_{m}\right) \backslash \underline{0} \rightarrow T^{*}\left({ }^{\mathbb{K}} G r_{k}\right) \backslash \underline{0}
$$

is a submersion. 
Proof. By taking the orthogonal complement if necessary, we may and assume that $k=1$. Recall that

$$
T^{*}\left({ }^{\mathbb{K}} G r_{k}\right)=\left\{(l, u) \mid l \in{ }^{\mathbb{K}} G r_{k}, u \in l \otimes_{\mathbb{K}} l^{\perp}\right\} .
$$

By Corollary 5.1 .2 we can identify

$$
T_{Z}^{*}\left({ }^{\mathbb{K}} G r_{k} \times{ }^{\mathbb{K}} G r_{m}\right) \simeq\left\{(l, H, v) \mid l \subset H, v \in l \otimes_{\mathbb{K}} H^{\perp}\right\} .
$$

Then the projection $f$ is given by

$$
f(l, H, v)=\left(l,\left(i d_{l} \otimes p^{*}\right)(v)\right)
$$

where, we remind, $i d_{l} \otimes p^{*}: l \otimes_{\mathbb{K}} H^{\perp} \rightarrow l \otimes_{\mathbb{K}} l^{\perp}$ is the natural imbedding.

Let us denote for brevity

$$
\begin{array}{r}
\left.S:=T_{Z}^{*}{ }^{\mathbb{K}} G r_{k} \times{ }^{\mathbb{K}} G r_{m}\right) \backslash \underline{0}, \\
\left.T:=T^{*}{ }^{\mathbb{K}} G r_{k}\right) \backslash \underline{0} .
\end{array}
$$

We have the commutative diagram

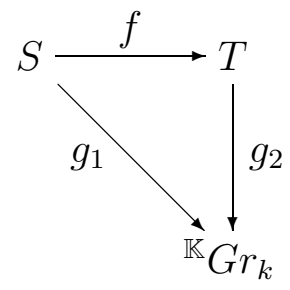

where $g_{1}, g_{2}$ are the obvious maps. Moreover all maps in this diagram commute with the natural action of the group $G L(n, \mathbb{K})$. Since the last group acts transitively on ${ }^{\mathbb{K}} G r_{k}$, it follows that $f: S \rightarrow T$ is a submersion if and only if for any (equivalently, for some) $l \in{ }^{\mathbb{K}} G r_{k}$ the restriction of $f$

$$
\left.f\right|_{g_{1}^{-1}(l)}: g_{1}^{-1}(l) \rightarrow g_{2}^{-1}(l)
$$

is a submersion. But for a fixed $l \in{ }^{\mathbb{K}} G r_{k}$ we have

$$
\begin{array}{r}
g_{1}^{-1}(l)=\left\{(H, v) \mid H \supset l, v \in\left(l \otimes_{\mathbb{K}} H^{\perp}\right) \backslash\{0\}\right\}, \\
g_{2}^{-1}(l)=\left(l \otimes_{\mathbb{K}} l^{\perp}\right) \backslash\{0\} .
\end{array}
$$

Then the restriction of $f$ to $g_{1}^{-1}(l)$ is given by

$$
f(H, v)=\left(i d_{l} \otimes p^{*}\right)(v) .
$$

Let us denote by $G \subset G L(n, \mathbb{K})$ the stabilizer of $l$. Since $k=1, G$ acts transitively on $g_{2}^{-1}(l)$. Moreover the map $f$ commutes with this action. This readily implies that $\left.f\right|_{g_{1}^{-1}(l)}: g_{1}^{-1}(l) \rightarrow g_{2}^{-1}(l)$ is a submersion. Hence $f$ is a submersion. Q.E.D. 


\section{References}

[1] Abardia, Judit; Gallego, Eduardo; Solanes, Gil; Gauss-Bonnet theorem and Crofton type formulas in complex space forms. Preprint: arXiv:0904.0336.

[2] Alesker, Semyon; Description of translation invariant valuations on convex sets with solution of P. McMullen's conjecture. Geom. Funct. Anal. 11 (2001), no. 2, 244-272.

[3] Alesker, Semyon; The multiplicative structure on polynomial valuations. Geom. Funct. Anal. 14 (2004), 1-26.

[4] Alesker, Semyon; Hard Lefschetz theorem for valuations, complex integral geometry, and unitarily invariant valuations. J. Differential Geom. 63 (2003), no. 1, 63-95. Also: math.MG/0209263.

[5] Alesker, Semyon; Theory of valuations on manifolds, I. Linear Spaces. Israel J. Math. 156 (2006), 311-340.

[6] Alesker, Semyon; Theory of valuations on manifolds, II. Adv. Math. 207 (2006), 420454.

[7] Alesker, Semyon; Theory of valuations on manifolds IV. New properties of the multiplicative structure. In Geometric aspects of functional analysis, LNM 1910 (2007), $1-44$.

[8] Alesker, Semyon; Theory of valuations on manifolds: a survey. Geom. Funct. Anal. 17 (2007), no. 4, 1321-1341. Also: math.MG/0603372.

[9] Alesker, Semyon; A Fourier type transform on translation invariant valuations on convex sets. Accepted to Israel J.Math., also: arXiv:math/0702842.

[10] Alesker, Semyon; On repeated sequential closures of constructible functions in valuations. Preprint arXiv:1408.2637

[11] Alesker, Semyon; Bernig, Andreas; The product on smooth and generalized valuations. Accepted to Amer. J. Math. Preprint, arXiv:0904.1347.

[12] Alesker, Semyon; Fu, Joseph H. G.; Theory of valuations on manifolds, III. Multiplicative structure in the general case. Trans. Amer. Math. Soc. 360 (2008), 1951-1981.

[13] Bernig, Andreas; A product formula for valuations on manifolds with applications to the integral geometry of the quaternionic line. Comm. Math. Helv. 84 (2009), 1-19. Also: arXiv:math/0611264.

[14] Bernig, Andreas; Bröcker, Ludwig; Valuations on manifolds and Rumin cohomology. J. Diff. Geom. 75 (2007), 433-457.

[15] Bernig, Andreas; Fu, Joseph H. G.; Hermitian integral geometry. Accepted to Ann. Math. Also: arXiv:0801.0711. 
[16] Bruce, J. W.; Giblin, P. J.; Curves and singularities. A geometrical introduction to singularity theory. Second edition. Cambridge University Press, Cambridge, 1992.

[17] Chern, Shiing-shen; On the curvatura integra in a Riemannian manifold. Ann. of Math. (2) 46, (1945). 674-684.

[18] Chern, Shiing-shen; On the kinematic formula in integral geometry. J. Math. Mech. 16 1966 101-118.

[19] Fu, Joseph H. G.; Curvature measures of subanalytic sets. Amer. J. Math. 116 (1994), no. $4,819-880$.

[20] Gelfand, I. M.; Graev, M. I.; Vilenkin, N. Ya.; Generalized functions. Vol. 5: Integral geometry and representation theory. Translated from the Russian by Eugene Saletan Academic Press, New York-London 1966.

[21] Gelfand, I. M.; Graev, M. I.; Roşu, R.; The problem of integral geometry and intertwining operators for a pair of real Grassmannian manifolds. J. Operator Theory 12 (1984), no. 2, 359-383.

[22] Guillemin, Victor; On some results of Gelfand in integral geometry. Pseudodifferential operators and applications (Notre Dame, Ind., 1984), 149-155, Proc. Sympos. Pure Math., 43, Amer. Math. Soc., Providence, RI, 1985.

[23] Guillemin, V., Sternberg, Sh.: Geometric asymptotics. Mathematical Surveys 14. American Mathematical Society (AMS), Providence, R.I. 1977.

[24] Hadwiger, Hugo; Vorlesungen über Inhalt, Oberfläche und Isoperimetrie. (German) Springer-Verlag, Berlin-Göttingen-Heidelberg 1957.

[25] Hörmander, Lars; The analysis of linear partial differential operators. I. Distribution theory and Fourier analysis. Reprint of the second (1990) edition [Springer, Berlin; MR1065993 (91m:35001a)]. Classics in Mathematics. Springer-Verlag, Berlin, 2003.

[26] Hörmander, Lars; Notions of convexity. Reprint of the 1994 edition. Modern Birkhäuser Classics. Birkhäuser Boston, Inc., Boston, MA, 2007.

[27] Kashiwara, Masaki; Schapira, Pierre; Sheaves on manifolds. Grundlehren der Mathematischen Wissenschaften, 292. Springer-Verlag, Berlin, 1990.

[28] Khovanskii, Askold G.; Pukhlikov, Alexander V.; Finitely additive measures of virtual polyhedra. (Russian) Algebra i Analiz 4 (1992), no. 2, 161-185; translation in St. Petersburg Math. J. 4 (1993), no. 2, 337-356

[29] Khovanskii, Askold G.; Pukhlikov, Alexander V.; Integral transforms based on Euler characteristic and their applications. Integral Transform. Spec. Funct. 1 (1993), no. 1, 19-26.

[30] McMullen, Peter; Valuations and dissections. Handbook of convex geometry, Vol. A, B, 933-988, North-Holland, Amsterdam, 1993. 
[31] McMullen, Peter; Schneider, Rolf; Valuations on convex bodies. Convexity and its applications, 170-247, Birkhäuser, Basel, 1983.

[32] Melrose, Richard; Differential analysis on manifolds with corners. http://math.mit.edu/ rbm/book.html

[33] Robertson, A. P.; Robertson, Wendy; Topological vector spaces. Second edition. Cambridge Tracts in Mathematics and Mathematical Physics, No. 53. Cambridge University Press, London-New York, 1973.

[34] Rumin, M.: Formes différentielles sur les variétés de contact. J. Differential Geom. 39 (1994), 281-330.

[35] Santaló, Luis A.; Integral geometry and geometric probability. With a foreword by Mark Kac. Encyclopedia of Mathematics and its Applications, Vol. 1. Addison-Wesley Publishing Co., Reading, Mass.-London-Amsterdam, 1976.

[36] Schaefer, Helmut H.; Topological vector spaces. Third printing corrected. Graduate Texts in Mathematics, Vol. 3. Springer-Verlag, New York-Berlin, 1971.

[37] Schapira, Pierre; Tomography of constructible functions. (English summary) Applied algebra, algebraic algorithms and error-correcting codes (Paris, 1995), 427-435, Lecture Notes in Comput. Sci., 948, Springer, Berlin, 1995.

[38] Schneider, Rolf; Convex Bodies: the Brunn-Minkowski Theory. Encyclopedia of Mathematics and its Applications, 44. Cambridge University Press, Cambridge, 1993.

[39] Viro, Oleg; Some integral calculus based on Euler characteristic. Topology and geometry-Rohlin Seminar, 127-138, Lecture Notes in Math., 1346, Springer, Berlin, 1988.

[40] Weyl, Hermann; On the volume of tubes. Amer.J.Math. 61 (1939), 461-472. 